\title{
High scale flavor alignment in two-Higgs doublet models and its phenomenology
}

\author{
Stefania Gori, ${ }^{a}$ Howard E. Haber ${ }^{b}$ and Edward Santos ${ }^{b}$ \\ ${ }^{a}$ Department of Physics, University of Cincinnati, \\ 345 Clifton Court, Cincinnati, OH 45221, U.S.A. \\ ${ }^{b}$ Santa Cruz Institute for Particle Physics, University of California, \\ 1156 High Street, Santa Cruz, CA 95064, U.S.A. \\ E-mail: stefania.gori@uc.edu, haber@scipp.ucsc.edu, \\ eddie.santos.3@gmail.com
}

ABSTRACT: The most general two-Higgs doublet model (2HDM) includes potentially large sources of flavor changing neutral currents (FCNCs) that must be suppressed in order to achieve a phenomenologically viable model. The flavor alignment ansatz postulates that all Yukawa coupling matrices are diagonal when expressed in the basis of mass-eigenstate fermion fields, in which case tree-level Higgs-mediated FCNCs are eliminated. In this work, we explore models with the flavor alignment condition imposed at a very high energy scale, which results in the generation of Higgs-mediated FCNCs via renormalization group running from the high energy scale to the electroweak scale. Using the current experimental bounds on flavor changing observables, constraints are derived on the aligned 2HDM parameter space. In the favored parameter region, we analyze the implications for Higgs boson phenomenology.

Keywords: Beyond Standard Model, Heavy Quark Physics, Higgs Physics

ArXiv EPrint: 1703.05873 


\section{Contents}

1 Introduction 1

2 The flavor-aligned 2HDM $\quad 4$

2.1 Theoretical framework for the 2HDM 4

2.2 The limit of a SM-like Higgs boson 9

2.3 Neutral scalars of definite CP 9

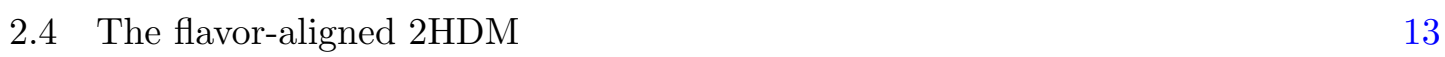

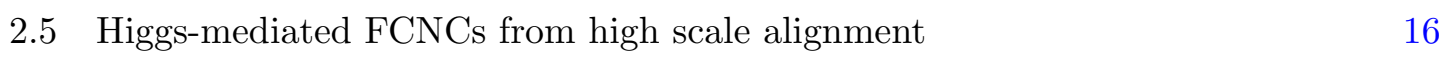

$\begin{array}{ll}2.6 & \text { Leading logarithm approximation } \\ & 18\end{array}$

$\begin{array}{ll}2.7 & \text { A particular type of Minimal Flavor Violation } \\ \end{array}$

3 Predictions of the model for high energy processes $\quad 20$

3.1 The couplings of the SM-like Higgs boson 20

$\begin{array}{lll}3.2 & \text { Flavor-changing top decays } & 23\end{array}$

$\begin{array}{ll}3.3 & \text { Phenomenology of the heavy Higgs bosons } \\ & 25\end{array}$

4 Predictions of the model for low energy processes 28

$\begin{array}{lll}4.1 & \text { Meson mixing } & 29\end{array}$

$4.2 \quad B_{s, d} \rightarrow \mu^{+} \mu^{-}$decays 31

$4.3 \quad B \rightarrow \tau \nu$ decays $\quad 35$

$\begin{array}{lll}5 & \text { Conclusions } & 37\end{array}$

A Review of the Higgs-fermion Yukawa couplings in the Higgs basis $\quad 40$

B Renormalization group equations for the Yukawa matrices 42

\section{Introduction}

With the discovery of a particle closely resembling the Standard Model (SM) Higgs boson at the Large Hadron Collider (LHC) [1-3], attention now turns to elucidating the dynamics of electroweak symmetry breaking. Many critical question still remain unanswered. What is the origin of the electroweak scale, and what mechanism ensures its stability? In light of the existence of multiple generations of fermions, are there also multiple copies of the scalar multiplets, implying the existence of additional Higgs scalars? If yes, how are the Higgs-fermion Yukawa interactions compatible with the apparent Minimal Flavor Violation (MFV), which is responsible for suppressed flavor changing neutral currents (FCNCs)?

Motivations for extending the Higgs sector beyond its minimal form have appeared often in the literature. For example, the minimal supersymmetric extension of the Standard 
Model, which is invoked to explain the stability of the electroweak symmetry breaking scale with respect to very high mass scales (such as the grand unification or Planck scales), requires a second Higgs doublet [4-7] to avoid anomalies due to the Higgsino partners of the Higgs bosons. More complicated scalar sectors may also be required for a realistic model of baryogenesis [8]. Finally, the metastability of the SM Higgs vacuum [9-11] can be rendered stable up to the Planck scale in models of extended Higgs sectors [12-19]. Even in the absence of a specific model of new physics beyond the Standard Model, an enlarged scalar sector can provide a rich phenomenology that can be probed by experimental searches now underway at the LHC.

One of the simplest extensions of the SM Higgs sector is the two-Higgs doublet model $(2 \mathrm{HDM}) .{ }^{1}$ In its most general form, the $2 \mathrm{HDM}$ is incompatible with experimental data due to the existence of unsuppressed tree-level Higgs-mediated FCNCs, in contrast to the SM where tree-level Higgs-mediated FCNCs are absent. To see why this is so, consider the Higgs-fermion Yukawa interactions expressed in terms of interaction eigenstate fermion fields. Due to the non-zero vacuum expectation value (vev) of the neutral Higgs field, fermion mass matrices are generated. Redefining the left and right-handed fermion fields by separate unitary transformations, the fermion mass matrices are diagonalized. In the SM, this diagonalization procedure also diagonalizes the neutral Higgs-fermion couplings, and consequently no tree-level Higgs-mediated FCNCs are present. In contrast, in a generic $2 \mathrm{HDM}$, the diagonalization of the fermion mass matrices implies the diagonalization of one linear combination of Higgs-fermion Yukawa coupling matrices. As a result, tree-level Higgs-mediated FCNCs remain in the 2HDM Lagrangian when expressed in terms of masseigenstate fermion fields. If it were possible in the 2HDM to realize flavor-diagonal neutral Higgs couplings at tree-level (thereby eliminating all tree-level Higgs-mediated FCNCs), then all FCNC processes arising in the model would be generated at the loop-level, with magnitudes more easily in agreement with experimental constraints. ${ }^{2}$

A natural mechanism for eliminating the tree-level Higgs-mediated FCNCs was proposed by Glashow and Weinberg [22] and by Paschos [23] [GWP]. One can implement the GWP mechanism in the $2 \mathrm{HDM}$ by introducing a $\mathbb{Z}_{2}$ symmetry to eliminate half of the Higgs-fermion Yukawa coupling terms. In this case, the fermion mass matrices and the non-zero Higgs-fermion Yukawa coupling matrices (which are consistent with the $\mathbb{Z}_{2}$ symmetry) are simultaneously diagonalized. Indeed, there are a number of inequivalent implementations of the GWP mechanism, resulting in the so-called Types I [24, 25], and II $[25,26]$, and Types $\mathrm{X}$ and $\mathrm{Y}[27,28]$ versions of the $2 \mathrm{HDM}^{3}$

\footnotetext{
${ }^{1}$ For a review with a comprehensive list of references, see ref. [20].

${ }^{2}$ Even in models with flavor-diagonal neutral Higgs couplings, one-loop processes mediated by the charged Higgs boson can generate significant FCNC effects involving third generation quarks. Such models, in order to be consistent with experimental data, will produce constraints in the $\left[m_{H^{ \pm}}, \tan \beta\right]$ plane. The most stringent constraint of this type, obtained in ref. [21] in the analysis of the Type-II 2HDM prediction for $b \rightarrow s \gamma$, yields $m_{H^{ \pm}} \gtrsim 580 \mathrm{GeV}$ at $95 \% \mathrm{CL}$.

${ }^{3}$ However, if additional degrees of freedom exist at the $\mathrm{TeV}$ scale, then the GWP mechanism is in general not sufficient to protect the theory from FCNCs that are incompatible with the experimental data. These $\mathrm{TeV}$-scale degrees of freedom, when integrated out, can generate higher-dimensional operators of the type $\left(c_{1} / \Lambda^{2}\right) \bar{Q}_{L} Y_{u 1}^{(6)} U_{R} H_{2}\left|H_{1}\right|^{2}+\cdots$, which break the proportionality relation between quark masses and effective Yukawa interactions with the neutral scalars. As a result, such models generically generate FCNC processes that are not sufficiently suppressed [29].
} 
Another strategy for eliminating tree-level Higgs-mediated FCNCs is by fiat. The flavor alignment ansatz proposed in ref. [30] asserts a proportionality between the two sets of Yukawa matrices. If this flavor-alignment condition is implemented at the electroweak scale, then the diagonalization of the fermion mass matrices simultaneously yields flavordiagonal neutral Higgs couplings. Moreover, this flavor-aligned 2HDM (henceforth denoted as the $\mathrm{A} 2 \mathrm{HDM}$ ) preserves the relative hierarchy in the quark mass matrices, and provides additional sources of CP-violation in the Yukawa Lagrangian via the introduction of three complex alignment parameters. Unfortunately, apart from the special cases enumerated in ref. [31], there are no symmetries within the $2 \mathrm{HDM}$ that guarantee the stability of the flavor alignment ansatz with respect to radiative corrections. As such, flavor alignment at the electroweak scale must be generically regarded as an unnatural fine-tuning of the Higgs-fermion Yukawa matrix parameters. Indeed, the Types I, II, X and Y 2HDMs are the unique special cases of flavor alignment that are radiatively stable after imposing the observed fermion masses and mixing [32].

In this paper, we consider the possibility that flavor alignment arises from New Physics beyond the 2HDM. Without a specific ultraviolet completion in mind, we shall assert that flavor alignment is imposed at some high energy scale, $\Lambda$, perhaps as large as a grand unification scale or the Planck scale, where new dynamics can emerge (e.g., see ref. [33] for a viable model). Once we impose the flavor alignment ansatz at the scale $\Lambda$, the effective field theory below this scale corresponds to a $2 \mathrm{HDM}$ with both Higgs doublets coupling to up type and down type quarks and leptons. ${ }^{4}$ We then employ renormalization group (RG) evolution to determine the structure of the 2HDM Yukawa couplings at the electroweak scale. For a generic flavor alignment ansatz at the scale $\Lambda$, flavor alignment in the Higgs-fermion Yukawa couplings at the electroweak scale is violated, thereby generating Higgs-mediated FCNCs. However, these FCNCs will be of Minimal Flavor Violation [35] type and therefore may be small enough to be consistent with experimental constraints, depending on the choice of the initial alignment parameters at the scale $\Lambda$.

We therefore examine the phenomenology of Higgs-mediated FCNCs that arise from the assumption of flavor alignment at some high energy scale, $\Lambda$, that, for the purpose of our analyses, is fixed to be the Planck scale $\left(M_{\mathrm{P}}\right)$. We note that similar work was performed in [36], where meson mixing and $B$ decays were used to constrain the A2HDM parameter space with flavor alignment at the Planck scale. Numerical results were obtained analytically in [36], using the leading logarithmic approximation. The results of this paper are first obtained in the leading log approximation, and then numerically by evolving the full one-loop renormalization group equations (RGEs) down from the Planck scale to the electroweak scale. In our work, we discuss the validity of the leading log approximation and examine additional FCNC processes at high energy (top and Higgs decays) and at low energy ( $B$ meson decays) to place bounds on the A2HDM parameters.

\footnotetext{
${ }^{4}$ In practice, one should also append to the 2HDM some mechanism for generating neutrino masses. An example of incorporating the effects of neutrino masses and mixing in the context of a $2 \mathrm{HDM}$ with flavor changing neutral Higgs couplings can be found in ref. [34]. In this paper, we shall simply put all neutrino masses to zero for the sake of simplicity. The extension of the results of this paper to models that incorporate a mechanism for neutrino mass generation will be considered in a future publication.
} 
This paper is organized as follows. In section 2, we review the theoretical framework of the general 2HDM. It is convenient to make use of the Higgs basis, which is unique up to a phase degree of freedom. All physical observables must be independent of this phase. In particular, we examine in detail the structure of the Higgs-fermion Yukawa couplings and exhibit its flavor structure. In the formalism presented in section 2, we initially allow for the most general form of the Higgs scalar potential and the Yukawa coupling matrices. In particular, new sources of CP-violation beyond the SM can arise due to unremovable complex phases in both the scalar potential parameters and the Yukawa couplings. For simplicity, we subsequently choose to analyze the case of a CP-conserving Higgs scalar potential and vacuum, in which case the neutral mass-eigenstates consist of two CP-even and one CP-odd neutral Higgs bosons. We then introduce the flavor-aligned 2HDM, in which the Yukawa coupling matrices are diagonal in the basis of quark and lepton mass-eigenstates. However, alignment is not stable under renormalization group running. Following the framework for flavor discussed above, we impose the alignment condition at the Planck scale and then evaluate the Yukawa coupling matrices of the Higgs basis at the electroweak scale as determined by renormalization group running, subject to the observed quark and lepton masses and the CKM mixing matrix. The renormalization group running is performed numerically and checked in the leading log approximation, where simple analytic expressions can be obtained. In this context, a comparison with general Minimal Flavor Violating 2HDMs is performed.

In section 3, we discuss the implications of high-scale flavor alignment for high energy processes. We focus on flavor-changing decays of the top quark and on the phenomenology of the heavy neutral CP-even and CP-odd Higgs bosons. In section 4, we discuss the implications of high-scale flavor alignment for low energy processes. Here we consider constraints arising from neutral meson mixing observables and from $B_{s} \rightarrow \ell^{+} \ell^{-}$, which receive contributions at tree-level from neutral Higgs exchange, and from the charged Higgs mediated $B \rightarrow \tau \nu$ decay. By comparing theoretical predictions to experimental data, one can already probe certain regions of the A2HDM parameter space. Additional parameter regions will be probed by future searches for heavy Higgs bosons and measurements of $B$-physics observables. Conclusions of this work are presented in section 5. Finally, in appendix A we review the derivation of the Yukawa sector of our model in the fermion mass-eigenstate basis, and in appendix B we exhibit the one-loop matrix Yukawa coupling RGEs used in this analysis.

\section{The flavor-aligned $2 \mathrm{HDM}$}

\subsection{Theoretical framework for the 2HDM}

Consider a generic $2 \mathrm{HDM}$ consisting of two complex, hypercharge-one scalar doublets, $\Phi_{1}$ and $\Phi_{2}$. The most general renormalizable scalar potential that is invariant under local $\mathrm{SU}(2) \times \mathrm{U}(1)$ gauge transformations can be written as

$$
\begin{aligned}
\mathcal{V}= & m_{11}^{2} \Phi_{1}^{\dagger} \Phi_{1}+m_{22}^{2} \Phi_{2}^{\dagger} \Phi_{2}-\left[m_{12}^{2} \Phi_{1}^{\dagger} \Phi_{2}+\text { h.c. }\right]+\frac{1}{2} \lambda_{1}\left(\Phi_{1}^{\dagger} \Phi_{1}\right)^{2}+\frac{1}{2} \lambda_{2}\left(\Phi_{2}^{\dagger} \Phi_{2}\right)^{2}+\lambda_{3}\left(\Phi_{1}^{\dagger} \Phi_{1}\right)\left(\Phi_{2}^{\dagger} \Phi_{2}\right) \\
& +\lambda_{4}\left(\Phi_{1}^{\dagger} \Phi_{2}\right)\left(\Phi_{2}^{\dagger} \Phi_{1}\right)+\left\{\frac{1}{2} \lambda_{5}\left(\Phi_{1}^{\dagger} \Phi_{2}\right)^{2}+\left[\lambda_{6}\left(\Phi_{1}^{\dagger} \Phi_{1}\right)+\lambda_{7}\left(\Phi_{2}^{\dagger} \Phi_{2}\right)\right] \Phi_{1}^{\dagger} \Phi_{2}+\text { h.c. }\right\} .
\end{aligned}
$$


The parameters of the scalar potential can be chosen so that the minimum of the scalar potential is achieved when the neutral components of the two scalar doublet fields acquire non-zero vacuum expectation vales, $\left\langle\Phi_{1}^{0}\right\rangle=v_{1} / \sqrt{2}$ and $\left\langle\Phi_{2}^{0}\right\rangle=v_{2} / \sqrt{2}$, where the (potentially complex) vevs satisfy

$$
v^{2} \equiv\left|v_{1}\right|^{2}+\left|v_{2}\right|^{2} \simeq(246 \mathrm{GeV})^{2},
$$

as required by the observed $W$ boson mass, $m_{W}=\frac{1}{2} g v$. The $\mathrm{SU}(2) \times \mathrm{U}(1)$ gauge symmetry is then spontaneously broken, leaving an unbroken $\mathrm{U}(1)_{\mathrm{EM}}$ gauge group.

In the most general $2 \mathrm{HDM}$, the fields $\Phi_{1}$ and $\Phi_{2}$ are indistinguishable. Thus, it is always possible to define two orthonormal linear combinations of the two doublet fields without modifying any prediction of the model. Performing such a redefinition of fields leads to a new scalar potential with the same form as eq. (2.1) but with modified coefficients. This implies that the coefficients that parameterize the scalar potential in eq. (2.1) are not directly physical [37].

To obtain a scalar potential that is more closely related to physical observables, one can introduce the so-called Higgs basis in which the redefined doublet fields (denoted below by $H_{1}$ and $H_{2}$ ) have the property that $H_{1}$ has a non-zero vev whereas $H_{2}$ has a zero vev $[37,38]$. In particular, we define the new Higgs doublet fields:

$$
H_{1}=\left(\begin{array}{c}
H_{1}^{+} \\
H_{1}^{0}
\end{array}\right) \equiv \frac{v_{1}^{*} \Phi_{1}+v_{2}^{*} \Phi_{2}}{v}, \quad H_{2}=\left(\begin{array}{c}
H_{2}^{+} \\
H_{2}^{0}
\end{array}\right) \equiv \frac{-v_{2} \Phi_{1}+v_{1} \Phi_{2}}{v} .
$$

It follows that $\left\langle H_{1}^{0}\right\rangle=v / \sqrt{2}$ and $\left\langle H_{2}^{0}\right\rangle=0$. The Higgs basis is uniquely defined up to an overall rephasing, $H_{2} \rightarrow e^{i \chi} H_{2}$ (which does not alter the fact that $\left\langle H_{2}^{0}\right\rangle=0$ ). In the Higgs basis, the scalar potential is given by $[37,38]$ :

$$
\begin{aligned}
\mathcal{V}= & Y_{1} H_{1}^{\dagger} H_{1}+Y_{2} H_{2}^{\dagger} H_{2}+\left[Y_{3} H_{1}^{\dagger} H_{2}+\text { h.c. }\right]+\frac{1}{2} Z_{1}\left(H_{1}^{\dagger} H_{1}\right)^{2}+\frac{1}{2} Z_{2}\left(H_{2}^{\dagger} H_{2}\right)^{2}+Z_{3}\left(H_{1}^{\dagger} H_{1}\right)\left(H_{2}^{\dagger} H_{2}\right) \\
& +Z_{4}\left(H_{1}^{\dagger} H_{2}\right)\left(H_{2}^{\dagger} H_{1}\right)+\left\{\frac{1}{2} Z_{5}\left(H_{1}^{\dagger} H_{2}\right)^{2}+\left[Z_{6}\left(H_{1}^{\dagger} H_{1}\right)+Z_{7}\left(H_{2}^{\dagger} H_{2}\right)\right] H_{1}^{\dagger} H_{2}+\text { h.c. }\right\},
\end{aligned}
$$

where $Y_{1}, Y_{2}$ and $Z_{1}, \ldots, Z_{4}$ are real and uniquely defined, whereas $Y_{3}, Z_{5}, Z_{6}$ and $Z_{7}$ are potentially complex and transform under the rephasing of $H_{2} \rightarrow e^{i \chi} H_{2}$ as

$$
\left[Y_{3}, Z_{6}, Z_{7}\right] \rightarrow e^{-i \chi}\left[Y_{3}, Z_{6}, Z_{7}\right] \text { and } Z_{5} \rightarrow e^{-2 i \chi} Z_{5}
$$

since $\mathcal{V}$ must be independent of $\chi$. After minimizing the scalar potential,

$$
Y_{1}=-\frac{1}{2} Z_{1} v^{2}, \quad Y_{3}=-\frac{1}{2} Z_{6} v^{2}
$$

This leaves 11 free parameters: 1 vev, 8 real parameters, $Y_{2}, Z_{1,2,3,4},\left|Z_{5,6,7}\right|$, and two relative phases.

In the general $2 \mathrm{HDM}$, the physical charged Higgs boson is the charged component of the Higgs-basis doublet $H_{2}$, and its mass is given by

$$
m_{H^{ \pm}}^{2}=Y_{2}+\frac{1}{2} Z_{3} v^{2}
$$


The three physical neutral Higgs boson mass-eigenstates are determined by diagonalizing a $3 \times 3$ real symmetric squared-mass matrix that is defined in the Higgs basis [38, 39]

$$
\mathcal{M}^{2}=v^{2}\left(\begin{array}{ccc}
Z_{1} & \operatorname{Re} Z_{6} & -\operatorname{Im} Z_{6} \\
\operatorname{Re} Z_{6} & \frac{1}{2}\left(Z_{345}+Y_{2} / v^{2}\right) & -\frac{1}{2} \operatorname{Im} Z_{5} \\
-\operatorname{Im} Z_{6} & -\frac{1}{2} \operatorname{Im} Z_{5} & \frac{1}{2}\left(Z_{345}+Y_{2} / v^{2}\right)-\operatorname{Re} Z_{5}
\end{array}\right)
$$

where $Z_{345} \equiv Z_{3}+Z_{4}+\operatorname{Re} Z_{5}$.

To identify the neutral Higgs mass-eigenstates, we diagonalize the squared-mass matrix $\mathcal{M}^{2}$. The diagonalization matrix is a $3 \times 3$ real orthogonal matrix that depends on three angles: $\theta_{12}, \theta_{13}$ and $\theta_{23}$. Following ref. [39],

$$
\left(\begin{array}{l}
h_{1} \\
h_{2} \\
h_{3}
\end{array}\right)=\left(\begin{array}{ccc}
c_{12} c_{13} & -s_{12} c_{23}-c_{12} s_{13} s_{23} & -c_{12} s_{13} c_{23}+s_{12} s_{23} \\
s_{12} c_{13} & c_{12} c_{23}-s_{12} s_{13} s_{23} & -s_{12} s_{13} c_{23}-c_{12} s_{23} \\
s_{13} & c_{13} s_{23} & c_{13} c_{23}
\end{array}\right)\left(\begin{array}{c}
\sqrt{2} \operatorname{Re} H_{1}^{0}-v \\
\sqrt{2} \operatorname{Re} H_{2}^{0} \\
\sqrt{2} \operatorname{Im} H_{2}^{0}
\end{array}\right),
$$

where the $h_{i}$ are the mass-eigenstate neutral Higgs fields, $c_{i j} \equiv \cos \theta_{i j}$ and $s_{i j} \equiv \sin \theta_{i j}$. Under the rephasing $\mathrm{H}_{2} \rightarrow e^{i \chi} \mathrm{H}_{2}$,

$$
\theta_{12}, \theta_{13} \text { are invariant, and } \theta_{23} \rightarrow \theta_{23}-\chi
$$

Assuming that $Z_{6} \equiv\left|Z_{6}\right| e^{i \theta_{6}} \neq 0,{ }^{5}$ it is convenient to define the invariant mixing angle,

$$
\phi \equiv \theta_{23}-\theta_{6}
$$

In light of the freedom to define the mass-eigenstate Higgs fields up to an overall sign, the invariant mixing angles $\theta_{12}, \theta_{13}$ and $\phi$ can be determined modulo $\pi$. By convention, we choose

$$
-\frac{1}{2} \pi \leq \theta_{12}, \theta_{13}<\frac{1}{2} \pi, \quad \text { and } \quad 0 \leq \phi<\pi .
$$

The physical neutral Higgs states $\left(h_{1,2,3}\right)$ are then given by:

$$
h_{k}=\frac{1}{\sqrt{2}}\left\{q_{k 1}^{*}\left(H_{1}^{0}-\frac{v}{\sqrt{2}}\right)+q_{k 2}^{*} H_{2}^{0} e^{i \theta_{23}}+\text { h.c. }\right\},
$$

where the $q_{k 1}$ and $q_{k 2}$ are invariant combinations of $\theta_{12}$ and $\theta_{13}$, which are exhibited in table 1 [39]. The masses of the neutral Higgs bosons $h_{i}$ will be denoted by $m_{i}$, respectively. It is convenient to define the physical charged Higgs states by

$$
H^{ \pm} \equiv e^{ \pm i \theta_{23}} H_{2}^{ \pm}
$$

so that all the Higgs mass-eigenstate fields $\left(h_{1}, h_{2}, h_{3}\right.$ and $\left.H^{ \pm}\right)$are invariant under $H_{2} \rightarrow e^{i \chi} H_{2}$.

\footnotetext{
${ }^{5}$ If $Z_{6}=0$, then one can always rephase the Higgs basis field $H_{2}$ such that $Z_{5}$ is real. In this basis, the neutral Higgs boson squared-mass matrix, $\mathcal{M}^{2}$, is diagonal, and the identification of the neutral Higgs boson mass-eigenstates is trivial.
} 


\begin{tabular}{|c|c|c|}
\hline$k$ & $q_{k 1}$ & $q_{k 2}$ \\
\hline 1 & $c_{12} c_{13}$ & $-s_{12}-i c_{12} s_{13}$ \\
2 & $s_{12} c_{13}$ & $c_{12}-i s_{12} s_{13}$ \\
3 & $s_{13}$ & $i c_{13}$ \\
\hline
\end{tabular}

Table 1. Invariant combinations of the neutral Higgs boson mixing angles $\theta_{12}$ and $\theta_{13}$, where $c_{i j} \equiv \cos \theta_{i j}$ and $s_{i j} \equiv \sin \theta_{i j}$.

Although the explicit formulae for the neutral Higgs boson masses and mixing angles are quite complicated, there are numerous relations among them which take on rather simple forms. The following results are noteworthy [39, 40]:

$$
\begin{aligned}
Z_{1} v^{2} & =m_{1}^{2} c_{12}^{2} c_{13}^{2}+m_{2}^{2} s_{12}^{2} c_{13}^{2}+m_{3}^{2} s_{13}^{2}, \\
\operatorname{Re}\left(Z_{6} e^{-i \theta_{23}}\right) v^{2} & =c_{13} s_{12} c_{12}\left(m_{2}^{2}-m_{1}^{2}\right), \\
\operatorname{Im}\left(Z_{6} e^{-i \theta_{23}}\right) v^{2} & =s_{13} c_{13}\left(c_{12}^{2} m_{1}^{2}+s_{12}^{2} m_{2}^{2}-m_{3}^{2}\right), \\
\operatorname{Re}\left(Z_{5} e^{-2 i \theta_{23}}\right) v^{2} & =m_{1}^{2}\left(s_{12}^{2}-c_{12}^{2} s_{13}^{2}\right)+m_{2}^{2}\left(c_{12}^{2}-s_{12}^{2} s_{13}^{2}\right)-m_{3}^{2} c_{13}^{2}, \\
\operatorname{Im}\left(Z_{5} e^{-2 i \theta_{23}}\right) v^{2} & =2 s_{12} c_{12} s_{13}\left(m_{2}^{2}-m_{1}^{2}\right) .
\end{aligned}
$$

We next turn to the Higgs-fermion Yukawa couplings. As reviewed in appendix A, one starts out initially with a Lagrangian expressed in terms of the scalar doublet fields $\Phi_{i}$ $(i=1,2)$ and interaction-eigenstate quark and lepton fields. After electroweak symmetry breaking, one can re-express the scalar doublet fields in terms of the Higgs basis fields $H_{1}$ and $\mathrm{H}_{2}$. At the same time, one can identify the $3 \times 3$ quark and lepton mass matrices. By redefining the left and right-handed quark and lepton fields appropriately, the quark and lepton mass matrices are transformed into diagonal form, where the diagonal elements are real and non-negative. The resulting Higgs-fermion Yukawa Lagrangian is given by in eq. (A.16) and is repeated here for the convenience of the reader [40],

$$
\begin{aligned}
-\mathscr{L}_{\mathrm{Y}}= & \bar{U}_{L}\left(\kappa^{U} H_{1}^{0 \dagger}+\rho^{U} H_{2}^{0 \dagger}\right) U_{R}-\bar{D}_{L} K^{\dagger}\left(\kappa^{U} H_{1}^{-}+\rho^{U} H_{2}^{-}\right) U_{R} \\
& +\bar{U}_{L} K\left(\kappa^{D \dagger} H_{1}^{+}+\rho^{D \dagger} H_{2}^{+}\right) D_{R}+\bar{D}_{L}\left(\kappa^{D \dagger} H_{1}^{0}+\rho^{D \dagger} H_{2}^{0}\right) D_{R} \\
& +\bar{N}_{L}\left(\kappa^{E \dagger} H_{1}^{+}+\rho^{E \dagger} H_{2}^{+}\right) E_{R}+\bar{E}_{L}\left(\kappa^{E \dagger} H_{1}^{0}+\rho^{D \dagger} H_{2}^{0}\right) E_{R}+\text { h.c. }
\end{aligned}
$$

where $U=(u, c, t)$ and $D=(d, s, b)$ are the mass-eigenstate quark fields, $K$ is the CKM mixing matrix, $N=\left(\nu_{e}, \nu_{\mu}, \nu_{\tau}\right)$ and $E=(e, \mu, \tau)$ are the mass-eigenstate lepton fields, and $\kappa$ and $\rho$ are $3 \times 3$ Yukawa coupling matrices. Note that $F_{R, L} \equiv P_{R, L} F$, where $F=U$, $D, N$ and $E$, and $P_{R, L} \equiv \frac{1}{2}\left(1 \pm \gamma_{5}\right)$ are the right and left-handed projection operators, respectively. At this stage, the neutrinos are exactly massless, so we are free to define the physical left-handed neutrino fields, $N_{L}$, such that their charged current interactions are generation-diagonal. ${ }^{6}$

By setting $H_{1}^{0}=v / \sqrt{2}$ and $H_{2}^{0}=0$, one can relate $\kappa^{U}, \kappa^{D}$, and $\kappa^{E}$ to the diagonal (up-type and down-type) quark and charged lepton mass matrices $M_{U}, M_{D}$, and $M_{E}$,

\footnotetext{
${ }^{6}$ To incorporate the neutrino masses, one can employ a seesaw mechanism [41-45] and introduce three right-handed neutrino fields along with an explicit $\mathrm{SU}(2) \times \mathrm{U}(1)$ conserving mass term. See footnote 4 .
} 
respectively,

$$
\begin{aligned}
& M_{U}=\frac{v}{\sqrt{2}} \kappa^{U}=\operatorname{diag}\left(m_{u}, m_{c}, m_{t}\right), \quad M_{D}=\frac{v}{\sqrt{2}} \kappa^{D \dagger}=\operatorname{diag}\left(m_{d}, m_{s}, m_{b}\right), \\
& M_{E}=\frac{v}{\sqrt{2}} \kappa^{E \dagger}=\operatorname{diag}\left(m_{e}, m_{\mu}, m_{\tau}\right) .
\end{aligned}
$$

However, the complex matrices $\rho^{F}(F=U, D, E)$ are unconstrained. Moreover, under the rephasing $\mathrm{H}_{2} \rightarrow e^{i \chi} H_{2}$, the Yukawa matrix acquires an overall phase, $\rho^{F} \rightarrow e^{i \chi} \rho^{F}$, since $\mathscr{L}_{\mathrm{Y}}$ must be independent of $\chi$.

To obtain the physical Yukawa couplings of the Higgs boson, one must relate the Higgs basis scalar fields to the Higgs mass-eigenstate fields. Using eqs. (2.13) and (2.14), the Higgs-fermion Yukawa couplings are given by,

$$
\begin{aligned}
-\mathscr{L}_{Y}= & \bar{U} \sum_{k=1}^{3}\left\{q_{k 1} \frac{M_{U}}{v}+\frac{1}{\sqrt{2}}\left[q_{k 2}^{*} e^{i \theta_{23}} \rho^{U} P_{R}+q_{k 2}\left[e^{i \theta_{23}} \rho^{U}\right]^{\dagger} P_{L}\right]\right\} U h_{k} \\
& +\bar{D} \sum_{k=1}^{3}\left\{q_{k 1} \frac{M_{D}}{v}+\frac{1}{\sqrt{2}}\left[q_{k 2}\left[e^{i \theta_{23}} \rho^{D}\right]^{\dagger} P_{R}+q_{k 2}^{*} e^{i \theta_{23}} \rho^{D} P_{L}\right]\right\} D h_{k} \\
& +\bar{E} \sum_{k=1}^{3}\left\{q_{k 1} \frac{M_{E}}{v}+\frac{1}{\sqrt{2}}\left[q_{k 2}\left[e^{i \theta_{23}} \rho^{E}\right]^{\dagger} P_{R}+q_{k 2}^{*} e^{i \theta_{23}} \rho^{E} P_{L}\right]\right\} E h_{k} \\
& +\left\{\bar{U}\left[K\left[e^{i \theta_{23}} \rho^{D}\right]^{\dagger} P_{R}-\left[e^{i \theta_{23}} \rho^{U}\right]^{\dagger} K P_{L}\right] D H^{+}+\bar{N}\left[e^{i \theta_{23}} \rho^{E}\right]^{\dagger} P_{R} E H^{+}+\text {h.c. }\right\} .
\end{aligned}
$$

The combinations $e^{i \theta_{23}} \rho^{U}, e^{i \theta_{23}} \rho^{D}$ and $e^{i \theta_{23}} \rho^{E}$ that appear in these interactions are invariant under the rephasing of $H_{2}$. It is convenient to rewrite the Higgs-fermion Yukawa couplings in terms of the following three $3 \times 3$ hermitian matrices that are invariant with respect to the rephasing of $\mathrm{H}_{2}$,

$$
\begin{array}{ll}
\rho_{R}^{F} \equiv \frac{v}{2 \sqrt{2}} M_{F}^{-1 / 2}\left\{e^{i \theta_{23}} \rho^{F}+\left[e^{i \theta_{23}} \rho^{F}\right]^{\dagger}\right\} M_{F}^{-1 / 2}, & \text { for } F=U, D, E, \\
\rho_{I}^{F} \equiv \frac{v}{2 \sqrt{2} i} M_{F}^{-1 / 2}\left\{e^{i \theta_{23}} \rho^{F}-\left[e^{i \theta_{23}} \rho^{F}\right]^{\dagger}\right\} M_{F}^{-1 / 2}, & \text { for } F=U, D, E,
\end{array}
$$

where the $M_{F}$ are the diagonal fermion mass matrices [cf. eq. (2.21)] and the Yukawa coupling matrices are introduced in eq. (2.20). Then, the Yukawa couplings take the following form:

$$
\begin{aligned}
-\mathscr{L}_{Y}= & \frac{1}{v} \bar{U} \sum_{k=1}^{3} M_{U}^{1 / 2}\left\{q_{k 1} \mathbb{1}+\operatorname{Re}\left(q_{k 2}\right)\left[\rho_{R}^{U}+i \gamma_{5} \rho_{I}^{U}\right]+\operatorname{Im}\left(q_{k 2}\right)\left[\rho_{I}^{U}-i \gamma_{5} \rho_{R}^{U}\right]\right\} M_{U}^{1 / 2} U h_{k} \\
& +\frac{1}{v} \bar{D} \sum_{k=1}^{3} M_{D}^{1 / 2}\left\{q_{k 1} \mathbb{1}+\operatorname{Re}\left(q_{k 2}\right)\left[\rho_{R}^{D}-i \gamma_{5} \rho_{I}^{D}\right]+\operatorname{Im}\left(q_{k 2}\right)\left[\rho_{I}^{D}+i \gamma_{5} \rho_{R}^{D}\right]\right\} M_{D}^{1 / 2} D h_{k} \\
& +\frac{1}{v} \bar{E} \sum_{k=1}^{3} M_{E}^{1 / 2}\left\{q_{k 1} \mathbb{1}+\operatorname{Re}\left(q_{k 2}\right)\left[\rho_{R}^{E}-i \gamma_{5} \rho_{I}^{E}\right]+\operatorname{Im}\left(q_{k 2}\right)\left[\rho_{I}^{E}+i \gamma_{5} \rho_{R}^{E}\right]\right\} M_{E}^{1 / 2} E h_{k} \\
& +\frac{\sqrt{2}}{v}\left\{\bar{U}\left[K M_{D}^{1 / 2}\left(\rho_{R}^{D}-i \rho_{I}^{D}\right) M_{D}^{1 / 2} P_{R}-M_{U}^{1 / 2}\left(\rho_{R}^{U}-i \rho_{I}^{U}\right) M_{U}^{1 / 2} K P_{L}\right] D H^{+}\right. \\
& \left.\bar{N} M_{E}^{1 / 2}\left(\rho_{R}^{E}-i \rho_{I}^{E}\right) M_{E}^{1 / 2} P_{R} E H^{+}+\text {h.c. }\right\},
\end{aligned}
$$


where $\mathbb{1}$ is the $3 \times 3$ identity matrix. The appearance of unconstrained hermitian $3 \times 3$ Yukawa matrices $\rho_{R, I}^{F}$ in eq. (2.25) indicates the presence of potential flavor-changing neutral Higgs-quark and lepton interactions. If the off-diagonal elements of $\rho_{R, I}^{F}$ are unsuppressed, they will generate tree-level Higgs-mediated FCNCs that are incompatible with the strong suppression of FCNCs observed in nature.

\subsection{The limit of a SM-like Higgs boson}

Current LHC data suggest that the properties of the observed Higgs boson are consistent with the predictions of the Standard Model. In this paper, we shall identify $h_{1}$ as the SM-like Higgs boson. In light of the expression for the $h_{1}$ coupling to a pair of vector bosons $V V=W^{+} W^{-}$or $Z Z$,

$$
\frac{g_{h_{1} V V}}{g_{h_{\mathrm{SM}} V V}}=c_{12} c_{13} \simeq 1, \quad \text { where } V=W \text { or } Z
$$

it follows that $\left|s_{12}\right|,\left|s_{13}\right| \ll 1$. Thus, in the limit of a SM-like Higgs boson, eqs. (2.16) and (2.17) yield [39]:

$$
\begin{aligned}
& \left|s_{12}\right| \simeq\left|\frac{\operatorname{Re}\left(Z_{6} e^{-i \theta_{23}}\right) v^{2}}{m_{2}^{2}-m_{1}^{2}}\right| \ll 1, \\
& \left|s_{13}\right| \simeq\left|\frac{\operatorname{Im}\left(Z_{6} e^{-i \theta_{23}}\right) v^{2}}{m_{3}^{2}-m_{1}^{2}}\right| \ll 1 .
\end{aligned}
$$

In addition, eq. (2.19) implies that one additional small quantity characterizes the limit of a SM-like Higgs boson,

$$
\left|\operatorname{Im}\left(Z_{5} e^{-2 i \theta_{23}}\right)\right| \simeq\left|\frac{2\left(m_{2}^{2}-m_{1}^{2}\right) s_{12} s_{13}}{v^{2}}\right| \simeq\left|\frac{\operatorname{Im}\left(Z_{6}^{2} e^{-2 i \theta_{23}}\right) v^{2}}{m_{3}^{2}-m_{1}^{2}}\right| \ll 1 .
$$

Moreover, in the limit of a SM-like Higgs boson, eq. (2.18) yields

$$
m_{2}^{2}-m_{3}^{2} \simeq \operatorname{Re}\left(Z_{5} e^{-2 i \theta_{23}}\right) v^{2}
$$

As a consequence of eqs. (2.27) and (2.28), the limit of a SM-like Higgs boson ${ }^{7}$ can be achieved if either $\left|Z_{6}\right| \ll 1$ and/or if $m_{2}, m_{3} \gg v$. The latter corresponds to the well-known decoupling limit of the $2 \mathrm{HDM}[39,46,53] .{ }^{8}$ In this paper, we will focus on the decoupling regime of the $2 \mathrm{HDM}$ to ensure that $h_{1}$ is sufficiently SM-like, in light of the current LHC Higgs data [3].

\subsection{Neutral scalars of definite CP}

In the exact SM-Higgs boson limit, the couplings of $h_{1}$ are precisely those of the SM Higgs boson. In this case, we can identify $h_{1}$ as a CP-even scalar. In general, the heavier neutral Higgs bosons, $h_{2}$ and $h_{3}$ can be mixed CP states. The limit in which $h_{2}$ and $h_{3}$

\footnotetext{
${ }^{7}$ In the literature, this is often referred to as the alignment limit [46-52]. We do not use this nomenclature here in order to avoid confusion with flavor alignment, which is the focus of this paper.

${ }^{8}$ Note that eq. (2.30) implies that in the decoupling limit, $m_{2} \gg v$ implies that $m_{3} \gg v$ and vice versa.
} 
are approximate eigenstates of $\mathrm{CP}$ is noteworthy. This limit is achieved assuming that $\left|s_{13}\right| \ll\left|s_{12}\right|$. That is,

$$
\left|\frac{s_{13}}{s_{12}}\right| \simeq\left|\left(\frac{m_{2}^{2}-m_{1}^{2}}{m_{3}^{2}-m_{1}}\right) \frac{\operatorname{Im}\left(Z_{6} e^{-i \theta_{23}}\right)}{\operatorname{Re}\left(Z_{6} e^{-i \theta_{23}}\right)}\right| \ll 1 .
$$

In the decoupling limit, the ratio of squared-mass differences in eq. (2.31) is of $\mathcal{O}(1)$. Moreover, unitarity and perturbativity constraints suggest that $\operatorname{Re}\left(Z_{6} e^{-i \theta_{23}}\right)$ cannot be significantly larger than $\mathcal{O}(1)$. Hence, it follows that

$$
\left|\operatorname{Im}\left(Z_{6} e^{-i \theta_{23}}\right)\right| \ll 1
$$

In light of eq. (2.10), we can rephase $H_{2} \rightarrow e^{i \chi} H_{2}$ such that $\theta_{23}=0(\bmod \pi)$, i.e. $c_{23}= \pm 1$. Eqs. (2.29) and (2.32) then yield $\left|\operatorname{Im} Z_{5}\right|,\left|\operatorname{Im} Z_{6}\right| \ll 1$. For simplicity in the subsequent analysis, we henceforth assume that a real Higgs basis exists in which $Z_{5}$ and $Z_{6}$ are simultaneously real. In this case, the scalar Higgs potential and the Higgs vacuum are CP-invariant, and the squared-mass matrix of the neutral Higgs bosons given in eq. (2.8) simplifies,

$$
\mathcal{M}^{2}=\left(\begin{array}{ccc}
Z_{1} v^{2} & Z_{6} v^{2} & 0 \\
Z_{6} v^{2} & Y_{2}+\frac{1}{2}\left(Z_{3}+Z_{4}+Z_{5}\right) v^{2} & 0 \\
0 & 0 & Y_{2}+\frac{1}{2}\left(Z_{3}+Z_{4}-Z_{5}\right) v^{2}
\end{array}\right),
$$

where $Z_{5}$ and $Z_{6}$ are real. Moreover, $c_{13}=1$ and we can set $\theta_{23}=\theta_{6}=0(\bmod \pi)$, or equivalently

$$
e^{i \theta_{23}}=c_{23}=\epsilon_{6},
$$

where $\epsilon_{6} \equiv \operatorname{sgn} Z_{6}$, in the real Higgs basis [cf. eqs. (2.11) and (2.12)]. To maintain the reality of the Higgs basis, the only remaining freedom in defining the Higgs basis fields is the overall sign of the field $H_{2}$. In particular, under $H_{2} \rightarrow-H_{2}$, we see that $Z_{5}$ is invariant whereas $Z_{6}$ (and $Z_{7}$ ) and $c_{23}$ change sign. We immediately identify the CP-odd Higgs boson $A=\sqrt{2} \operatorname{Im} H_{2}^{0}$ with squared mass,

$$
m_{A}^{2}=Y_{2}+\frac{1}{2}\left(Z_{3}+Z_{4}-Z_{5}\right) v^{2} .
$$

Note that the real Higgs mass-eigenstate field, $A$, is defined up to an overall sign change, which corresponds to the freedom to redefine $\mathrm{H}_{2} \rightarrow-H_{2}$. In contrast, the charged Higgs field $H^{ \pm}$defined (as a matter of convenience) by eq. (2.14) is invariant with respect to $H_{2} \rightarrow-H_{2}$. Indeed, by using eq. (2.34), we can now write $H^{ \pm}=\epsilon_{6} H_{2}^{ \pm}$. In light of eqs. (2.7) and (2.35),

$$
m_{H^{ \pm}}^{2}=m_{A}^{2}-\frac{1}{2}\left(Z_{4}-Z_{5}\right) v^{2}
$$

The upper $2 \times 2$ matrix block given in eq. (2.33) is the CP-even Higgs squared-mass matrix,

$$
\mathcal{M}_{H}^{2}=\left(\begin{array}{cc}
Z_{1} v^{2} & Z_{6} v^{2} \\
Z_{6} v^{2} & m_{A}^{2}+Z_{5} v^{2}
\end{array}\right)
$$


where we have used eq. (2.35) to eliminate $Y_{2}$. To diagonalize $\mathcal{M}_{H}^{2}$, we define the CP-even mass-eigenstates, $h$ and $H$ (with $m_{h} \leq m_{H}$ ) by

$$
\left(\begin{array}{l}
H \\
h
\end{array}\right)=\left(\begin{array}{cr}
c_{\beta-\alpha} & -s_{\beta-\alpha} \\
s_{\beta-\alpha} & c_{\beta-\alpha}
\end{array}\right)\left(\begin{array}{c}
\sqrt{2} \operatorname{Re} H_{1}^{0}-v \\
\sqrt{2} \operatorname{Re} H_{2}^{0}
\end{array}\right),
$$

where $c_{\beta-\alpha} \equiv \cos (\beta-\alpha)$ and $s_{\beta-\alpha} \equiv \sin (\beta-\alpha)$ are defined in terms of the angle $\beta$ defined via $\tan \beta \equiv v_{2} / v_{1}$, and the mixing angle $\alpha$ that diagonalizes the CP-even Higgs squaredmass matrix when expressed relative to the original basis of scalar fields, $\left\{\Phi_{1}, \Phi_{2}\right\}$, which is assumed here to be a real basis. ${ }^{9}$ Since the real Higgs mass-eigenstate fields $H$ and $h$ are defined up to an overall sign change, it follows that $\beta-\alpha$ is determined modulo $\pi$. To make contact with the notation of eq. (2.9), we note that $c_{13}=1$ and $c_{23}=\epsilon_{6}$ [cf. eq. (2.34)]. Assuming that $h_{1}$ is the lighter of the two neutral CP-even Higgs bosons, then eq. (2.38) implies the following identifications:

$$
h=h_{1}, \quad H=-\epsilon_{6} h_{2}, \quad A=\epsilon_{6} h_{3},
$$

and

$$
c_{12}=s_{\beta-\alpha}, \quad s_{12}=-\epsilon_{6} c_{\beta-\alpha} .
$$

This means that the signs of the fields $H$ and $A$ and the sign of $c_{\beta-\alpha}$ all flip under the redefinition of the Higgs basis field $H_{2} \rightarrow-H_{2}$.

Note that $0 \leq s_{\beta-\alpha} \leq 1$ in the convention specified in eq. (2.12). Moreover, eq. (2.16) yields

$$
s_{\beta-\alpha} c_{\beta-\alpha}=-\frac{Z_{6} v^{2}}{m_{H}^{2}-m_{h}^{2}},
$$

and it therefore follows that $0 \leq s_{12}, c_{12} \leq 1$ and $c_{\beta-\alpha} Z_{6} \leq 0$. The decoupling limit corresponds to $m_{H} \gg m_{h}$ and $\left|c_{\beta-\alpha}\right| \ll 1$ [cf. eq. (2.27)], in which case we can identify $h$ as the SM-like Higgs boson and $H$ as the heavier CP-even Higgs boson. Finally, eqs. (2.15)(2.19) yield

$$
\begin{aligned}
& Z_{1} v^{2}=m_{h}^{2} s_{\beta-\alpha}^{2}+m_{H}^{2} c_{\beta-\alpha}^{2}, \\
& Z_{6} v^{2}=\left(m_{h}^{2}-m_{H}^{2}\right) s_{\beta-\alpha} c_{\beta-\alpha}, \\
& Z_{5} v^{2}=m_{H}^{2} s_{\beta-\alpha}^{2}+m_{h}^{2} c_{\beta-\alpha}^{2}-m_{A}^{2} .
\end{aligned}
$$

In particular, $m_{h}^{2} \simeq Z_{1} v^{2}$ in the limit of a SM-like Higgs boson $h$. Applying eq. (2.40) to table 1,

$$
\begin{array}{ll}
q_{11}=s_{\beta-\alpha}, & q_{12}=\epsilon_{6} c_{\beta-\alpha}, \\
q_{21}=-\epsilon_{6} c_{\beta-\alpha}, & q_{22}=s_{\beta-\alpha}, \\
q_{31}=0, & q_{32}=i .
\end{array}
$$

\footnotetext{
${ }^{9}$ Given the assumption [indicated above eq. (2.33)] that the scalar Higgs potential and the Higgs vacuum are CP-invariant, it follows that there must exist a real basis of scalar fields in which all scalar potential parameters and the vacuum expectation values of the two neutral Higgs fields, $\left\langle\Phi_{i}^{0}\right\rangle \equiv v_{i} / \sqrt{2}$ (for $i=1,2$ ), are simultaneously real [54].
} 
Inserting these results into the general form of the Yukawa couplings given in eq. (2.25), we obtain the following Higgs-fermion couplings in the case of a CP-conserving Higgs scalar potential and vacuum,

$$
\begin{aligned}
&-\mathscr{L}_{Y}= \frac{1}{v} \sum_{F=U, D, E} \bar{F}\left\{s_{\beta-\alpha} M_{F}+\epsilon_{6} c_{\beta-\alpha} M_{F}^{1 / 2}\left[\rho_{R}^{F}+i \varepsilon_{F} \gamma_{5} \rho_{I}^{F}\right] M_{F}^{1 / 2}\right\} F h \\
&+\frac{1}{v} \sum_{F=U, D, E} \bar{F}\left\{c_{\beta-\alpha} M_{F}-\epsilon_{6} s_{\beta-\alpha} M_{F}^{1 / 2}\left[\rho_{R}^{F}+i \varepsilon_{F} \gamma_{5} \rho_{I}^{F}\right] M_{F}^{1 / 2}\right\} F H \\
&+\frac{1}{v} \sum_{F=U, D, E} \bar{F}\left\{M_{F}^{1 / 2} \epsilon_{6}\left(\rho_{I}^{F}-i \varepsilon_{F} \gamma_{5} \rho_{R}^{F}\right) M_{F}^{1 / 2}\right\} F A \\
&+\frac{\sqrt{2}}{v}\left\{\bar{U}\left[K M_{D}^{1 / 2}\left(\rho_{R}^{D}-i \rho_{I}^{D}\right) M_{D}^{1 / 2} P_{R}-M_{U}^{1 / 2}\left(\rho_{R}^{U}-i \rho_{I}^{U}\right) M_{U}^{1 / 2} K P_{L}\right] D H^{+}\right. \\
&\left.\bar{N} M_{E}^{1 / 2}\left(\rho_{R}^{E}-i \rho_{I}^{E}\right) M_{E}^{1 / 2} P_{R} E H^{+}+\text {h.c. }\right\}
\end{aligned}
$$

where we have introduced the notation,

$$
\varepsilon_{F}= \begin{cases}+1 & \text { for } F=U, \\ -1 & \text { for } F=D, E\end{cases}
$$

Moreover, by employing eq. (2.34) in eqs. (2.23) and (2.24), the expressions for $\rho_{R}^{F}$ and $\rho_{I}^{F}$ in terms of the Higgs Yukawa coupling matrices $\rho^{F}$ simplify,

$$
\begin{aligned}
\epsilon_{6} M_{F}^{1 / 2} \rho_{R}^{F} M_{F}^{1 / 2} & =\frac{v}{2 \sqrt{2}}\left(\rho^{F}+\left[\rho^{F}\right]^{\dagger}\right), \\
i \epsilon_{6} M_{F}^{1 / 2} \rho_{I}^{F} M_{F}^{1 / 2} & =\frac{v}{2 \sqrt{2}}\left(\rho^{F}-\left[\rho^{F}\right]^{\dagger}\right) .
\end{aligned}
$$

The structure of the neutral Higgs couplings given in eq. (2.48) is easily ascertained. If $\rho_{I}^{F} \neq 0$, then the neutral Higgs fields will exhibit CP-violating Yukawa couplings. ${ }^{10}$ Moreover, the two sign choices, $\epsilon_{6}= \pm 1$ are physically indistinguishable, since the sign of $Z_{6}$ can always be flipped by redefining the Higgs basis field $H_{2} \rightarrow-H_{2}$. Under this field redefinition, $\rho^{F}, c_{\beta-\alpha}, H$ and $A$ also flip sign, in which case $\mathscr{L}_{Y}$ is unchanged.

For completeness, we briefly consider the case where $h_{1}$ is the heavier of the two neutral CP-even Higgs bosons. In this case, eq. (2.38) implies the following identifications,

$$
h=\epsilon_{6} h_{2}, \quad H=h_{1}, \quad A=\epsilon_{6} h_{3},
$$

and

$$
c_{12}=c_{\beta-\alpha}, \quad s_{12}=\epsilon_{6} s_{\beta-\alpha} .
$$

This means that the signs of the fields $h$ and $A$ and the sign of $s_{\beta-\alpha}$ all flip under the redefinition of the Higgs basis field $H_{2} \rightarrow-H_{2}$. Note that eqs. (2.41)-(2.44) are still valid.

\footnotetext{
${ }^{10}$ Likewise, if $\operatorname{Im} Z_{7} \neq 0$ in a basis where $Z_{5}$ and $Z_{6}$ are real, then the neutral Higgs fields will also possess $\mathrm{CP}$-violating trilinear and quadralinear scalar couplings.
} 
Invoking the convention given by eq. (2.12) now implies that $0 \leq c_{\beta-\alpha} \leq 1$ and $Z_{6} s_{\beta-\alpha} \leq 0$. Moreover in light of eq. (2.26), if $\left|s_{\beta-\alpha}\right| \ll 1$ then $H$ is SM-like and $m_{H}^{2} \simeq Z_{1} v^{2}$, which is achieved in the limit of $\left|Z_{6}\right| \ll 1$. No decoupling limit is possible in this case since $m_{h}<m_{H}=125 \mathrm{GeV}$. Using eq. (2.53), one can check that eqs. (2.45)-(2.47) are modified by taking $s_{\beta-\alpha} \rightarrow c_{\beta-\alpha}$ and $c_{\beta-\alpha} \rightarrow-s_{\beta-\alpha}$. As a result, eq. (2.48) remains unchanged.

So far, the parameters $\alpha$ and $\beta$ have no separate significance. Only the combination, $\beta-\alpha$ is meaningful. Moreover the matrices $\rho_{R}^{F}$ and $\rho_{I}^{F}$ are generic complex matrices, which implies the existence of tree-level Higgs-mediated flavor changing neutral currents, as well as new sources of CP violation. However, experimental data suggest that such Higgs-mediated FCNCs must be highly suppressed. One can eliminate these FCNCs by imposing a discrete $\mathbb{Z}_{2}$ symmetry $\Phi_{1} \rightarrow \Phi_{1}$ and $\Phi_{2} \rightarrow-\Phi_{2}$ on the quartic terms of the Higgs potential given in eq. (2.1), which sets $\lambda_{6}=\lambda_{7}=0$ and gives physical significance to the $\Phi_{1}-\Phi_{2}$ basis choice. This in turn promotes the CP-even Higgs mixing angle $\alpha$ in the real $\Phi_{1}-\Phi_{2}$ basis and $\tan \beta \equiv v_{2} / v_{1}$ to physical parameters of the model. ${ }^{11}$ The $\mathbb{Z}_{2}$ symmetry can be extended to the Higgs-fermion interactions in four inequivalent ways. In the notation of the Higgs-fermion Yukawa couplings given in eq. (2.48), the $\rho_{R, I}^{F}$ are given by ${ }^{12}$

1. Type-I: for $F=U, D, E, \rho_{R}^{F}=\epsilon_{6} \cot \beta \mathbb{1}$ and $\rho_{I}^{F}=0$.

2. Type-II: $\rho_{R}^{U}=\epsilon_{6} \cot \beta \mathbb{1}$ and $\rho_{I}^{U}=0$. For $F=D, E, \rho_{R}^{F}=-\epsilon_{6} \tan \beta \mathbb{1}$ and $\rho_{I}^{F}=0$.

3. Type-X: $\rho_{R}^{E}=-\epsilon_{6} \tan \beta \mathbb{1}$ and $\rho_{I}^{E}=0$. For $F=U, D, \rho_{R}^{F}=\epsilon_{6} \cot \beta \mathbb{1}$ and $\rho_{I}^{F}=0$.

4. Type-Y: $\rho_{R}^{D}=-\epsilon_{6} \tan \beta \mathbb{1}$ and $\rho_{I}^{D}=0$. For $F=U, E, \rho_{R}^{F}=\epsilon_{6} \cot \beta \mathbb{1}$ and $\rho_{I}^{F}=0$.

Inserting these values for the $\rho_{R}^{F}$ and $\rho_{I}^{F}$ into eq. (2.48), the resulting neutral Higgs-fermion Yukawa couplings are flavor diagonal as advertised.

From a purely phenomenological point of view, one can simply avoid tree-level Higgsmediated FCNCs by declaring that the $\rho_{R}^{F}$ and $\rho_{I}^{F}$ are diagonal matrices. In the simplest generalization of the Type I, II, X and Y Yukawa interactions, one asserts that both the $\rho_{R}^{F}$ and the $\rho_{I}^{F}$ are proportional to the identity matrix (where the constants of proportionality can depend on $F$ ). This is called the flavor-aligned 2HDM, which we shall discuss in the next subsection.

\subsection{The flavor-aligned $2 \mathrm{HDM}$}

The flavor-aligned 2HDM posits that the Yukawa matrices $\kappa^{F}$ and $\rho^{F}$ [cf. eq. (2.20)] are proportional. When written in terms of fermion mass-eigenstates, $\kappa^{F}=\sqrt{2} M_{F} / v$ is diagonal. Thus in the A2HDM, the $\rho^{F}$ are likewise diagonal, which implies that tree-level

\footnotetext{
${ }^{11}$ Since the existence of a real Higgs basis implies no spontaneous nor explicit CP-violation in the scalar sector, there exists a $\Phi_{1}-\Phi_{2}$ basis in which the $\lambda_{i}$ of eq. (2.1), $v_{1}$ and $v_{2}$ (and hence $\tan \beta$ ) are simultaneously real.

${ }^{12}$ As defined here, the parameter $\tan \beta$ flips sign under the redefinition of the Higgs basis field $H_{2} \rightarrow-H_{2}$, in contrast to the more common convention where $\tan \beta$ is positive (by redefining $H_{2} \rightarrow-H_{2}$ if necessary). With this latter definition, the two cases of $\epsilon_{6}= \pm 1$ [or equivalently the two cases of $\operatorname{sgn}\left(s_{\beta-\alpha} c_{\beta-\alpha}\right)=\mp 1$ ] represent non-equivalent points of the Type-I, II, X or Y 2 HDM parameter space. However, we do not adopt this latter convention in the present work.
} 
Higgs-mediated FCNCs are absent. We define the alignment parameters $a^{F}$ via,

$$
\rho^{F}=e^{-i \theta_{23}} a^{F} \kappa^{F}, \quad \text { for } F=U, D, E,
$$

where the (potentially) complex numbers $a^{F}$ are invariant under the rephasing of the Higgs basis field $H_{2} \rightarrow e^{i \chi} H_{2}$. It follows from eqs. (2.23) and (2.24) that

$$
\rho_{R}^{F}=\left(\operatorname{Re} a^{F}\right) \mathbb{1}, \quad \rho_{I}^{F}=\left(\operatorname{Im} a^{F}\right) \mathbb{1} .
$$

Inserting the above results into eq. (2.22), the Yukawa couplings take the following form:

$$
\begin{aligned}
-\mathscr{L}_{Y}= & \frac{1}{v} \bar{U} \sum_{k=1}^{3} M_{U}\left\{q_{k 1}+q_{k 2}^{*} a^{U} P_{R}+q_{k 2} a^{U *} P_{L}\right\} U h_{k} \\
& +\frac{1}{v} \bar{D} \sum_{k=1}^{3} M_{D}\left\{q_{k 1}+q_{k 2} a^{D *} P_{R}+q_{k 2}^{*} a^{D} P_{L}\right\} D h_{k} \\
& +\frac{1}{v} \bar{E} \sum_{k=1}^{3} M_{E}\left\{q_{k 1}+q_{k 2} a^{E *} P_{R}+q_{k 2}^{*} a^{E} P_{L}\right\} E h_{k} \\
& +\frac{\sqrt{2}}{v}\left\{\bar{U}\left[a^{D *} K M_{D} P_{R}-a^{U *} M_{U} K P_{L}\right] D H^{+}+a^{E *} \bar{N} M_{E} P_{R} E H^{+}+\text {h.c. }\right\} .
\end{aligned}
$$

This form simplifies further if the neutral Higgs mass-eigenstates are also states of definite CP. In this case, the corresponding Yukawa couplings are given by

$$
\begin{aligned}
-\mathscr{L}_{Y}= & \frac{1}{v} \sum_{F=U, D, E} \bar{F} M_{F}\left\{s_{\beta-\alpha}+\epsilon_{6} c_{\beta-\alpha}\left[\operatorname{Re} a^{F}+i \epsilon^{F} \operatorname{Im} a^{F} \gamma_{5}\right]\right\} F h \\
& +\frac{1}{v} \sum_{F=U, D, E} \bar{F} M_{F}\left\{c_{\beta-\alpha}-\epsilon_{6} s_{\beta-\alpha}\left[\operatorname{Re} a^{F}+i \epsilon^{F} \operatorname{Im} a^{F} \gamma_{5}\right]\right\} F H \\
& +\frac{1}{v} \sum_{F=U, D, E} \bar{F} M_{F}\left\{\epsilon_{6}\left[\operatorname{Im} a^{F}-i \epsilon^{F} \operatorname{Re} a^{F} \gamma_{5}\right]\right\} F A \\
& +\frac{\sqrt{2}}{v}\left\{\bar{U}\left[a^{D *} K M_{D} P_{R}-a^{U *} M_{U} K P_{L}\right] D H^{+}+a^{E *} \bar{N} M_{E} P_{R} E H^{+}+\text {h.c. }\right\} .
\end{aligned}
$$

As noted above eq. (2.41), it is convenient to choose a convention in which $s_{\beta-\alpha} \geq 0$. It then follows from eq. (2.41) that $\epsilon_{6} c_{\beta-\alpha}=-\left|c_{\beta-\alpha}\right|$. That is, the neutral Higgs couplings exhibited in eq. (2.57) do not depend on the sign of $c_{\beta-\alpha}$ (which can be flipped by redefining the overall sign of the Higgs basis field $H_{2}$ ). Note that in this convention, the signs of the alignment parameters $a^{F}$ are physical.

The Type-I, II, X and Y Yukawa couplings are special cases of the A2HDM Yukawa couplings. Since the $a^{F}(F=U, D, E)$ are independent complex numbers, there is no preferred basis for the scalar fields outside of the Higgs basis. Thus, a priori, there is no separate meaning to the parameters $\alpha$ and $\beta$ in eq. (2.57). Nevertheless, in the special case of a CP-conserving neutral Higgs-lepton interaction governed by eq. (2.57) with $\operatorname{Im} a^{E}=0$, it is convenient to introduce the real parameter $\tan \beta$ via

$$
a^{E} \equiv-\epsilon_{6} \tan \beta,
$$


corresponding to a Type-II or Type-X Yukawa couplings of the charged leptons to the neutral Higgs bosons. The theoretical interpretation of $\tan \beta$ defined by eq. (2.58) is as follows. It is always possible to choose a $\Phi_{1}-\Phi_{2}$ basis with the property that one of the two Higgs-lepton Yukawa coupling matrices vanishes. Namely, in the notation of eq. (A.1), we have $\eta_{2}^{E, 0}=0$, which means that only $\Phi_{1}$ couples to leptons. In the case of a CP-conserving scalar Higgs potential and Higgs vacuum, we can take the $\Phi_{1}-\Phi_{2}$ basis to be a real basis and identify $\tan \beta=v_{2} / v_{1}$, where $\left\langle\Phi_{i}^{0}\right\rangle \equiv v_{i} / \sqrt{2}$ (for $i=1,2$ ). However, in contrast to Type-II or Type-X models, $\eta_{2}^{E, 0}=0$ does not correspond to a discrete $\mathbb{Z}_{2}$ symmetry of the generic A2HDM Lagrangian, since we do not require any of the Higgs-quark Yukawa coupling matrices and the scalar potential parameters $\lambda_{6}$ and $\lambda_{7}$ to vanish in the same $\Phi_{1}-\Phi_{2}$ basis.

Note that the sign of $a^{E}$ in eq. (2.58) is physical since both $\epsilon_{6}$ and $\tan \beta$ flip sign under the Higgs basis field $H_{2} \rightarrow-H_{2}$. In contrast to the standard conventions employed in the 2HDM with Type-I, II, X or Y Yukawa couplings where $\tan \beta$ is defined to be positive [cf. footnote 12], we shall not adopt such a convention here. In practice, we will rewrite eq. (2.58) as,

$$
a^{E}=\epsilon_{E}|\tan \beta|,
$$

where $\epsilon_{E}= \pm 1$ correspond to physically non-equivalent points of the A2HDM parameter space.

One theoretical liability of the A2HDM is that for generic choices of the alignment parameters $a^{U}$ and $a^{D}$, the flavor-alignment conditions in the quark sector specified in eq. (2.54) are not stable under the evolution governed by the Yukawa coupling renormalization group equations. Indeed, as shown in ref. [32], eq. (2.54) is stable under renormalization group running if and only if the parameters $a^{U}$ and $a^{D}$ satisfy the conditions of the Type I, II, X or Y 2HDMs specified at the end of section 2.3. In the leptonic sector, since we ignore neutrino masses, the Higgs-lepton Yukawa couplings are flavor-diagonal at all scales. We therefore assume that ${ }^{13}$

$$
\rho^{F}(\Lambda)=a^{F} \kappa^{F}(\Lambda), \quad \text { for } F=U, D
$$

at some very high energy scale $\Lambda$ (such as the grand unification (GUT) scale or the Planck scale). That is, we assume that the alignment conditions are set by some a priori unknown physics at or above the energy scale $\Lambda$. We take the complex alignment parameters $a^{F}$ to be boundary conditions for the RGEs of the Yukawa coupling matrices, and then determine the low-energy values of the Yukawa coupling matrices by numerically solving the RGEs. To ensure that the resulting low-energy theory is consistent with a SM-like Higgs boson observed at the LHC, we shall take $m_{h}=125 \mathrm{GeV}$, and assume that the masses of $H, A$ and $H^{ \pm}$are all of order $\Lambda_{H} \geq 400 \mathrm{GeV}$. In this approximate decoupling regime, $\left|c_{\beta-\alpha}\right|$ is small enough such that the properties of $h$ are within about $20 \%$ of the SM Higgs boson, as required by the LHC Higgs data [3]. We employ the 2HDM RGEs given in appendix B from $\Lambda$ down to $\Lambda_{H}$, and then match onto the RGEs of the Standard Model to generate the Higgs-fermion Yukawa couplings at the electroweak scale, which we take to be $m_{t}$ or $m_{Z}$. Note that the values of $\kappa^{Q}\left(\Lambda_{H}\right)=\sqrt{2} M_{Q}\left(\Lambda_{H}\right) / v$ (for $\left.Q=U, D\right)$ are determined from the known quark masses via Standard Model RG running.

\footnotetext{
${ }^{13}$ Under the assumption of a real Higgs basis, $\epsilon_{6}=e^{i \theta_{23}}$ is fixed via eq. (2.58). This factor, which appears in eq. (2.54), can then be absorbed into the definition of $a^{F}$.
} 
As noted above for the lepton case $(F=E)$, if $\rho^{E}(\Lambda)$ is proportional to $\kappa^{E}(\Lambda)$, then $\rho$ is proportional to $\kappa$ at all energy scales. Thus, we identify the leptonic alignment parameter at low energies by $\tan \beta$. More precisely [cf. eqs. (2.21) and (2.59)],

$$
\rho^{E}\left(\Lambda_{H}\right)=\sqrt{2} \epsilon_{E}|\tan \beta| M_{E}\left(\Lambda_{H}\right) / v .
$$

Then, $M_{E}\left(\Lambda_{H}\right)$ is determined by the diagonal lepton mass matrix via Standard Model RG running.

\subsection{Higgs-mediated FCNCs from high scale alignment}

To explore the Higgs-mediated FCNCs that can be generated in the A2HDM at the electroweak scale, we establish flavor-alignment at some high energy scale, $\Lambda$, as for example at the GUT or Planck scale, and run the one-loop RGEs from the high scale to the electroweak scale. Thus, we impose the following boundary conditions for the running of the one-loop 2HDM Yukawa couplings,

$$
\begin{aligned}
\kappa^{Q}\left(\Lambda_{H}\right) & =\sqrt{2} M_{Q}\left(\Lambda_{H}\right) / v \\
\rho^{Q}(\Lambda) & =a^{Q} \kappa^{Q}(\Lambda),
\end{aligned}
$$

where the $M_{Q}(Q=U, D)$ are the diagonal quark matrices, and $\Lambda_{H}$ is the scale of the heavier doublet, taken to be relatively large to guarantee that we are sufficiently in the decoupling limit. For the lepton sector, the corresponding boundary conditions are [cf. eq. (2.59)],

$$
\begin{aligned}
\kappa^{E}\left(\Lambda_{H}\right) & =\sqrt{2} M_{E}\left(\Lambda_{H}\right) / v, \\
\rho^{E}\left(\Lambda_{H}\right) & =\epsilon_{E}|\tan \beta| \kappa^{E}\left(\Lambda_{H}\right) .
\end{aligned}
$$

Satisfying the two boundary conditions for the quark sector [eqs. (2.62) and (2.63)] is not trivial, since they are imposed at opposite ends of the RG running. For example, to set flavor-alignment at the high energy scale, we must know the values of $\kappa^{Q}(\Lambda)$. This involves running up $\kappa^{Q}\left(\Lambda_{H}\right)$ to the high scale, but since the one-loop RGEs are strongly coupled to the $\rho^{Q}$ matrices, we must supply values for $\rho^{Q}\left(\Lambda_{H}\right)$ to begin the running.

With no a priori knowledge of which values of $\rho^{Q}\left(\Lambda_{H}\right)$ lead to flavor-alignment at the high scale, we begin the iterative process by assuming flavor-alignment at $\Lambda_{H}$ via a low-scale alignment parameter $a^{\prime Q}$,

$$
\rho^{Q}\left(\Lambda_{H}\right)=a^{\prime Q} \kappa^{Q}\left(\Lambda_{H}\right) .
$$

This flavor-alignment will be broken during RGE evolution to the high scale, and a procedure is needed to reestablish flavor-alignment at the high scale. To accomplish this, we decompose $\rho^{Q}(\Lambda)$ into parts that are aligned and misaligned with $\kappa^{Q}(\Lambda)$, respectively,

$$
\rho^{Q}(\Lambda)=a^{Q} \kappa^{Q}(\Lambda)+\delta \rho^{Q},
$$

where $a^{Q}$ represents the aligned part (in general, different from $a^{\prime Q}$ ), and $\delta \rho^{Q}$ the corresponding degree of misalignment at the high scale. 
To minimize the misaligned part of $\rho^{Q}(\Lambda)$, we implement the cost function,

$$
\Delta^{Q} \equiv \sum_{i, j=1}^{3}\left|\delta \rho_{i j}^{Q}\right|^{2}=\sum_{i, j=1}^{3}\left|\rho_{i j}^{Q}(\Lambda)-a^{Q} \kappa_{i j}^{Q}(\Lambda)\right|^{2},
$$

which, once minimized, provides the optimal value of the complex parameter $a^{Q}$ for flavoralignment at the high scale,

$$
a^{Q} \equiv \frac{\sum_{i, j=1}^{3} \kappa_{i j}^{Q *}(\Lambda) \rho_{i j}^{Q}(\Lambda)}{\sum_{i, j=1}^{3} \kappa_{i j}^{Q *}(\Lambda) \kappa_{i j}^{Q}(\Lambda)} .
$$

We subsequently impose flavor-alignment at the high scale using this optimized alignment parameter,

$$
\rho^{Q}(\Lambda)=a^{Q} \kappa^{Q}(\Lambda)
$$

and evolve the one-loop RGEs back down to $\Lambda_{H}$. In principle, further running of $\kappa^{U}$ and $\kappa^{D}$ below $\Lambda_{H}$ can regenerate off-diagonal terms. However, these effects are extremely small and can be ignored in practice. At $\Lambda_{H}$, we use (2.62) to match the boundary conditions for the $2 \mathrm{HDM}$ and SM. At this point, the matrices $\kappa^{U}$ and $\kappa^{D}$ at the scale $\Lambda_{H}$ are no longer diagonal, so we must rediagonalize $\kappa^{U}$ and $\kappa^{D}$ in analogy with eq. (A.12) [while respectively transforming $\rho^{U}$ and $\rho^{D}$ (at the scale $\Lambda_{H}$ ) in analogy with eq. (A.13)]. We can now evolve $\kappa^{U}$ and $\kappa^{D}$ down to the electroweak scale to check the accuracy of the resulting quark masses. If any of the quark masses differ from their experimental values by more than $3 \%$, we reestablish the correct quark masses at the electroweak scale, ${ }^{14}$ run back up to $\Lambda_{H}$, and then rerun this procedure repeatedly until the two boundary conditions are satisfied. The result is flavor-alignment between $\kappa^{Q}(\Lambda)$ and $\rho^{Q}(\Lambda)$, and a set of $\rho^{Q}$ matrices at the electroweak scale that provide a source of FCNCs.

In our iterative procedure, we demand that all scale-dependent Yukawa couplings remain finite from the electroweak scale to the Planck scale (i.e., Landau poles are absent below $\Lambda=M_{\mathrm{P}}$ ). This restricts the range of the possible seed values, $a^{\prime Q}$, used in eq. (2.66) to initialize the iteration. Consequently, the alignment parameters $a^{U}$ and $a^{D}$ cannot be too large in absolute value. Constraints on the alignment parameters due to Landau pole considerations during one-loop RG running have been given in ref. [59]. In our analysis, the allowed values of $a^{U}$ and $a^{D}$ consistent with the absence of Landau poles at all scales below $\Lambda$ are exhibited in figure $1 .^{15}$ Assuming $\Lambda_{H}=400 \mathrm{GeV}$, these considerations lead to bounds on the alignment parameters evaluated at the Planck scale, $\Lambda=M_{\mathrm{P}}$,

$$
\left|a^{U}\right| \lesssim 0.8 \text { and }\left|a^{D}\right| \lesssim 80
$$

which are consistent with the results previously obtained in ref. [59].

\footnotetext{
${ }^{14}$ Starting the RG evolution at $m_{Z}$, we use a five flavor scheme to run up to $m_{t}$ and a six flavor scheme above $m_{t}$. Running quark mass masses at $m_{Z}$ and $m_{t}$ are obtained from the RunDec Mathematica software package [55, 56], based on quark masses provided in ref. [57]. We fix the initial value of the top Yukawa coupling $y_{t}\left(m_{t}\right)=0.94$, corresponding to an $\overline{\mathrm{MS}}$ top quark mass of $m_{t}\left(m_{t}\right)=163.64 \mathrm{GeV}[58]$. For simplicity, the effects of the lepton masses are ignored, as these contribute very little to the running.

${ }^{15}$ If a Landau pole in one of the Yukawa coupling matrices arises at the scale $\Lambda$, then both the corresponding $\rho^{Q}(\Lambda)$ and $\kappa^{Q}(\Lambda)$ diverge, whereas their ratio, $a^{Q}$, remains finite.
} 


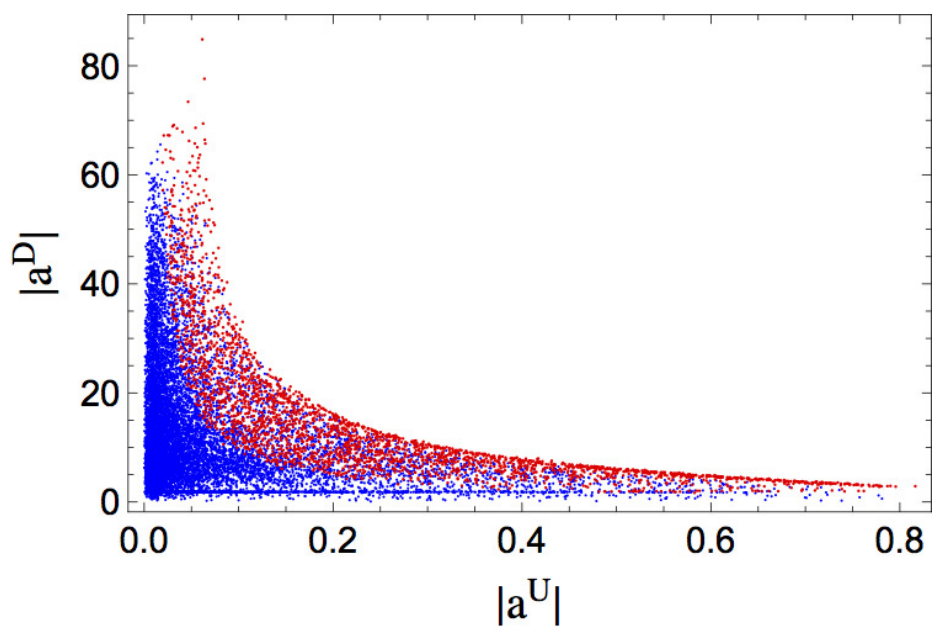

Figure 1. The allowed values of $a^{U}$ and $a^{D}$ consistent with the absence of Landau poles below $\Lambda=M_{\mathrm{P}}$ are exhibited. The blue points occupy the region of the A2HDM parameter space where the prediction for all entries of the $\rho^{Q}$ matrices lie within a factor of 3 from the results obtained with the full running. The red points occupy the region where the leading log approximation yields results quite different from the full RG running.

\subsection{Leading logarithm approximation}

In the limit of small alignment parameters, it is possible to obtain approximate analytic solutions to the one-loop RGEs provided in appendix B. One can express the $\rho^{Q}$ matrices at the low scale as

$$
\begin{aligned}
& \rho^{U}\left(\Lambda_{H}\right) \simeq a^{U} \kappa^{U}\left(\Lambda_{H}\right)+\frac{1}{16 \pi^{2}} \log \left(\frac{\Lambda_{H}}{\Lambda}\right)\left(\mathcal{D} \rho^{U}-a^{U} \mathcal{D} \kappa^{U}\right), \\
& \rho^{D}\left(\Lambda_{H}\right) \simeq a^{D} \kappa^{D}\left(\Lambda_{H}\right)+\frac{1}{16 \pi^{2}} \log \left(\frac{\Lambda_{H}}{\Lambda}\right)\left(\mathcal{D} \rho^{D}-a^{D} \mathcal{D} \kappa^{D}\right),
\end{aligned}
$$

where $D \kappa^{D}, D \kappa^{U}, D \rho^{D}, D \rho^{U}$ are the $\beta$-functions defined in eqs. (B.10)-(B.14) and $\kappa^{U}\left(\Lambda_{H}\right)$ and $\kappa^{D}\left(\Lambda_{H}\right)$ are proportional to the diagonal quark mass matrices, $M_{U}$ and $M_{D}$ respectively, at the scale $\Lambda_{H}$, according to eq. (2.21). Working to one loop order and neglecting higher order terms, it is consistent to set $\rho^{F}=a^{F} \kappa^{F}=a^{F} \sqrt{2} M_{F} / v$ (for $F=U, D, E)$ in the corresponding $\beta$-functions, ${ }^{16}$

$$
\begin{aligned}
\rho^{U}\left(\Lambda_{H}\right)_{i j} \simeq & a^{U} \delta_{i j} \frac{\sqrt{2}\left(M_{U}\right)_{j j}}{v}+\frac{\left(M_{U}\right)_{j j}}{4 \sqrt{2} \pi^{2} v^{3}} \log \left(\frac{\Lambda_{H}}{\Lambda}\right)\left\{\left(a^{E}-a^{U}\right)\left[1+a^{U}\left(a^{E}\right)^{*}\right] \delta_{i j} \operatorname{Tr}\left(M_{E}^{2}\right)\right. \\
& \left.+\left(a^{D}-a^{U}\right)\left[1+a^{U}\left(a^{D}\right)^{*}\right]\left[3 \delta_{i j} \operatorname{Tr}\left(M_{D}^{2}\right)-2 \sum_{k}\left(M_{D}^{2}\right)_{k k} K_{i k} K_{j k}^{*}\right]\right\}, \quad(2.74) \\
\rho^{D}\left(\Lambda_{H}\right)_{i j} \simeq & a^{D} \delta_{i j} \frac{\sqrt{2}\left(M_{D}\right)_{i i}}{v}+\frac{\left(M_{D}\right)_{i i}}{4 \sqrt{2} \pi^{2} v^{3}} \log \left(\frac{\Lambda_{H}}{\Lambda}\right)\left\{\left(a^{E}-a^{D}\right)\left[1+a^{D}\left(a^{E}\right)^{*}\right] \delta_{i j} \operatorname{Tr}\left(M_{E}^{2}\right)\right. \\
& \left.+\left(a^{U}-a^{D}\right)\left[1+a^{D}\left(a^{U}\right)^{*}\right]\left[3 \delta_{i j} \operatorname{Tr}\left(M_{U}^{2}\right)-2 \sum_{k}\left(M_{U}^{2}\right)_{k k} K_{k i}^{*} K_{k j}\right]\right\} .
\end{aligned}
$$

\footnotetext{
${ }^{16}$ The misalignment contributions exhibited in eqs. (2.74) and (2.75) were computed for the first time in ref. [60].
} 
It follows that there is a large hierarchy among the several off-diagonal terms of the $\rho^{Q}$ matrices,

$$
\begin{aligned}
\left|\frac{\rho^{D}\left(\Lambda_{H}\right)_{i j}}{\rho^{D}\left(\Lambda_{H}\right)_{j i}}\right| \sim \frac{\left(M_{D}\right)_{i i}}{\left(M_{D}\right)_{j j}} \ll 1, \quad \text { for } i<j, \\
\left|\frac{\rho^{U}\left(\Lambda_{H}\right)_{i j}}{\rho^{U}\left(\Lambda_{H}\right)_{j i}}\right| \sim \frac{\left(M_{U}\right)_{j j}}{\left(M_{U}\right)_{i i}} \gg 1, \quad \text { for } i<j .
\end{aligned}
$$

The inequality given in eq. (2.76) was previously noted in ref. [36], and provides the justification for ignoring $\rho_{i j}^{D}$ relative to $\rho_{j i}^{D}$, for $i<j .{ }^{17}$ This hierarchy of Yukawa couplings is reversed for $\rho_{i j}^{U}$. This reversal can be traced back to the fact that $\rho^{U}$ is undaggered in eq. (2.20) whereas $\rho^{D}$ is daggered.

It is noteworthy that the leading log results for the off-diagonal terms of the $\rho^{Q}$ matrices obtained in eqs. (2.74) and (2.75) and the corresponding full numerical calculation are typically within a factor of a few. Even for small alignment parameters, there can be some small discrepancies between the two approaches that can be traced back to the higher order terms that were neglected in eqs. (2.74) and (2.75). These higher order terms are not negligible due to the running performed between the electroweak scale and the high energy scale $\Lambda$. The leading log approximation describes less and less accurately the numerical results at larger and larger alignment parameters. This is shown in figure 1, where the blue points correspond to the parameter regime in which the leading log approach leads to results within a factor of 3 of the results obtained numerically for all the elements of the $\rho^{U}$ and $\rho^{D}$ matrices. In contrast, the red points correspond to the parameter regime in which the leading log approximation leads to results quite different from what is obtained by the full running.

\subsection{A particular type of Minimal Flavor Violation}

In the quark sector of the A2HDM, only the two Yukawa coupling matrices $\kappa^{U}$ and $\kappa^{D}$ break the $\mathrm{SU}(3)_{Q} \times \mathrm{SU}(3)_{U} \times \mathrm{SU}(3)_{D}$ global flavor symmetry of the electroweak Lagrangian involving quarks. For this reason, our model can be thought in terms of a specific realization of a Minimal Flavor Violating (MFV) 2HDM [29]. In particular, in a general 2HDM with MFV one can write the Yukawa Lagrangian as

$$
-\mathscr{L}_{\mathrm{Y}, \mathrm{MFV}}=\bar{Q}_{L} Y_{u} U_{R} H_{1}^{\dagger}+\bar{Q}_{L} Y_{d}^{\dagger} D_{R} H_{1}+\bar{Q}_{L} A_{u} U_{R} H_{2}^{\dagger}+\bar{Q}_{L} A_{d}^{\dagger} D_{R} H_{2}+\text { h.c., }
$$

with $H_{1}, H_{2}$ the two Higgs doublets in the Higgs basis as defined in section 2 and $Q_{L}, U_{R}, D_{R}$ flavor eigenstate quarks. In general, $A_{u}, A_{d}$ can be expressed by the infinite sum [35]

$$
\begin{aligned}
A_{u} & =\sum_{n_{1}, n_{2}, n_{3}} \epsilon_{n_{1} n_{2} n_{3}}^{u}\left(Y_{d} Y_{d}^{\dagger}\right)^{n_{1}}\left(Y_{u} Y_{u}^{\dagger}\right)^{n_{2}}\left(Y_{d} Y_{d}^{\dagger}\right)^{n_{3}} Y_{u} \\
A_{d} & =\sum_{n_{1}, n_{2}, n_{3}} \epsilon_{n_{1} n_{2} n_{3}}^{d}\left(Y_{d} Y_{d}^{\dagger}\right)^{n_{1}}\left(Y_{u} Y_{u}^{\dagger}\right)^{n_{2}}\left(Y_{d} Y_{d}^{\dagger}\right)^{n_{3}} Y_{d}
\end{aligned}
$$

with generic $\mathcal{O}(1)$ complex coefficients $\epsilon_{n i}^{u, d}$. In order to determine the coefficients $\epsilon_{n i}^{u, d}$ in the A2HDM, we rotate to the quark mass-eigenstate basis: $Y_{u} \rightarrow \kappa^{U}, A_{u} \rightarrow \rho^{U}, Y_{d} \rightarrow \kappa^{D}$,

\footnotetext{
${ }^{17}$ To make contact with the Higgs basis Yukawa couplings $\Delta_{u}$ and $\Delta_{d}$ employed by ref. [36], we note the relations $\rho^{U}=\sqrt{2} \Delta_{u}$ and $\rho^{D}=\sqrt{2} \Delta_{d}^{\dagger}$.
} 
$A_{d} \rightarrow \rho^{D}$ and compare with the leading log expressions for $\rho^{U}$ and $\rho^{D}$ as reported in eqs. (2.74) and (2.75). We find

$$
\begin{aligned}
& \epsilon_{000}^{u}=a^{U}-\frac{1}{8 \pi^{2} v^{2}} \log \left(\frac{\Lambda_{H}}{\Lambda}\right)\{ 3\left(a^{U}-a^{D}\right)\left[1+a^{U}\left(a^{D}\right)^{*}\right] \operatorname{Tr}\left(M_{D}^{2}\right) \\
&\left.+\left(a^{U}-a^{E}\right)\left[1+a^{U}\left(a^{E}\right)^{*}\right] \operatorname{Tr}\left(M_{E}^{2}\right)\right\}, \\
& \epsilon_{100}^{u}=\frac{1}{8 \pi^{2}} \log \left(\frac{\Lambda_{H}}{\Lambda}\right)\left(a^{U}-a^{D}\right)\left[1+a^{U}\left(a^{D}\right)^{*}\right],
\end{aligned}
$$

and all the higher order coefficients equal to zero. The corresponding coefficients for the down sector are obtained from these expressions with the replacement $a^{U} \rightarrow a^{D}, a^{D} \rightarrow$ $a^{U}, \kappa^{D} \rightarrow \kappa^{U}$. As expected, the leading term in eq. (2.81) is given by the alignment parameter at the high scale $a^{U}$. This coefficient receives one loop corrections. The term in eq. (2.82) generates off diagonal terms in the matrix $\rho^{U}$ and is one loop suppressed.

\section{Predictions of the model for high energy processes}

For our numerical analysis, we use the procedure described in the previous section, taking the A2HDM to be in the decoupling limit, which ensures that the properties of the lightest Higgs boson, $h$, are approximately those of the observed (SM-like) Higgs boson. As stated below eq. (2.33), we assume that the Higgs scalar potential and the Higgs vacuum are CPconserving. In this case, the two heavier neutral scalars, $H$ and $A$, are CP-even and CP-odd mass-eigenstates, respectively. In the decoupling limit, these two scalars are roughly degenerate in mass, i.e., $m_{H} \approx m_{A} \approx \Lambda_{H} \gg m_{h}$. The decoupling limit also enforces the condition $|\cos (\beta-\alpha)| \ll 1$, as noted below eq. (2.41). In this paper, we shall choose a benchmark mass of $m_{H}=400 \mathrm{GeV}$. Noting that in the case of a SM-like Higgs boson, $m_{h}^{2} \simeq Z_{1} v^{2}=$ $(125 \mathrm{GeV})^{2}$, which implies that $Z_{1} \simeq 0.26$, we will furthermore assume that $\left|Z_{6}\right|$ and $Z_{1}$ are of similar size. Indeed, eq. (2.41) yields $|\cos (\beta-\alpha)| \simeq 0.11$ for $\left|Z_{6}\right|=Z_{1} \cdot{ }^{18}$ In particular, if $\beta-\alpha=\pi / 2-x$, with $|x| \ll 1$, then values $x \neq 0$ imply deviations from SM behavior of the couplings of the $125 \mathrm{GeV}$ Higgs boson to fermions and gauge bosons, as well as the appearance of flavor changing neutral Higgs couplings, the largest of which is the hbs coupling.

In our analysis, we allow for CP-violating effects to enter in two ways. First, CPviolating charged Higgs couplings to fermion pairs are generated via the appearance of the CKM matrix, $K$. Second, we generically allow for the possibility of complex alignment parameters $a^{U}$ and $a^{D}$ at the high energy scale. Via RG-running, CP-violating neutral Higgs couplings to fermion pairs will be generated. However, this extra source of CP violation will lead to a loop-suppressed mixing of $H$ and $A$ that is difficult to observe due to the near mass degeneracy of these states in the decoupling limit under consideration here.

\subsection{The couplings of the SM-like Higgs boson}

It is instructive to examine the $h b \bar{b}$ coupling, which is the Yukawa coupling that is most affected by New Physics in our framework, and thus plays the leading role in constraining

\footnotetext{
${ }^{18}$ For $m_{H}=400 \mathrm{GeV}$, even a value as large as $\left|Z_{6}\right|=1$, yields $|\cos (\beta-\alpha)|=0.477$ [cf. eq. (2.41)], which is (barely) consistent with the measured $W W$ and $Z Z$ couplings of the observed Higgs boson.
} 
the parameter space. Following the standard notation of the ATLAS and CMS Collaborations, we denote the coupling of $h$ to bottom quarks normalized to the SM prediction by $\kappa_{b} .{ }^{19}$ Due to the presence of a CP-violating contribution to the $h b \bar{b}$ coupling when $\operatorname{Im} \rho_{33}^{D} \neq 0$, both scalar and pseudoscalar contributions to the $h b \bar{b}$ coupling must be considered [see eq. (2.48)]. In the approximation where $m_{b} \ll m_{h}$, one can simply replace $\gamma_{5}$ in the expression for the Yukawa coupling with \pm 1 , in which case $\kappa_{b}$ can be expressed by the magnitude of the complex number,

$$
\kappa_{b}=\left|s_{\beta-\alpha}+\frac{v}{m_{b} \sqrt{2}} c_{\beta-\alpha} \rho_{33}^{D}\right|,
$$

and compared to its ATLAS and CMS measurement, extracted from the $h \rightarrow b \bar{b}$ rate. In the leading log approximation, eq. (2.75) yields,

$$
\begin{aligned}
\kappa_{b}=\mid s_{\beta-\alpha} & +a^{D} c_{\beta-\alpha}+\frac{1}{8 \pi v^{2}} \log \left(\frac{\Lambda_{H}}{\Lambda}\right)\left\{\left(a^{E}-a^{D}\right)\left[1+a^{D}\left(a^{E}\right)^{*}\right] \operatorname{Tr}\left(M_{E}^{2}\right)\right. \\
& \left.+\left(a^{U}-a^{D}\right)\left[1+a^{D}\left(a^{U}\right)^{*}\right]\left[3 \operatorname{Tr}\left(M_{U}^{2}\right)-2 \sum_{k}\left(M_{U}^{2}\right)_{k k} K_{k 3}^{*} K_{k 3}\right]\right\} \mid .
\end{aligned}
$$

In figure 2 , we show the reduced coupling, $\kappa_{b}$, in the leading log approximation as a function of the free parameters $a^{U}, a^{D}$ and $|\cos (\beta-\alpha)|$. We extend the plots up to $|\cos (\beta-\alpha)| \sim 0.3$, consistent with the present measurement of the Higgs couplings to $W W$ and $Z Z$. The two upper panels are obtained using the leading log approximation; the two lower ones using the full RG running. We take real values for $a^{U}, a^{D}$ to present the leading log results. Generic complex coefficients are employed in parameter scans obtained with the full running. In the left upper panel, we show the reduced coupling as a function of $a^{U}$ and $a^{D}$, having fixed $|\cos (\beta-\alpha)|=0.05$. In the right upper panel, we show the reduced coupling as a function of $|\cos (\beta-\alpha)|$ and $a^{D}$, having fixed $a^{U}=0.1$. In the two panels, we show in blue the contour $\kappa_{b}=1.3$, that roughly corresponds to the present $3 \sigma$ bound, as measured by the LHC combining ATLAS and CMS Run I data [3]. ${ }^{20}$ The pink regions of figure 2 illustrate that values for $|\cos (\beta-\alpha)| \sim \mathcal{O}(0.1)$ are still allowed for sizable values of $a^{D}$ of $\mathcal{O}(20)$. Furthermore, the shape of the constraint is quite different, if compared to the shape obtained for the $(\cos (\beta-\alpha), \tan \beta)$ plane in the Type I and II 2HDM [61, 62]. The corresponding results obtained using the full RG running are shown in the lower panels. Note that the bounds on the parameter spaces $\left(\left|a^{U}\right|,\left|a^{D}\right|\right)$ and $\left(|\cos (\beta-\alpha)|,\left|a^{D}\right|\right)$ are slightly weaker as compared to the leading log results.

It is interesting to investigate the Higgs flavor violating couplings in the regions of parameter space favored by the LHC measurements of the SM Higgs rates. The decay to a bottom and a strange quarks is the dominant flavor violating Higgs decay in our model. However, we have checked that the corresponding branching ratio can be at most at the few per-mille level. Numerically, this is similar to the result for $\operatorname{BR}(h \rightarrow \bar{b} s+b \bar{s})$ obtained by ref. [63] in a Type I and Type II 2HDM due to charged Higgs loop contributions to the decay amplitude.

\footnotetext{
${ }^{19}$ Note that $\kappa_{b}$ should not be confused with the matrices $\kappa^{F}(F=U, D, E)$ defined in eqs. $(2.20)$ and (2.21).

${ }^{20}$ Under the assumption of no decay modes of the Higgs boson beyond the SM and no non-SM particles in the loop, ref. [3] obtains $\kappa_{b}=0.67_{-0.20}^{+0.22}$.
} 

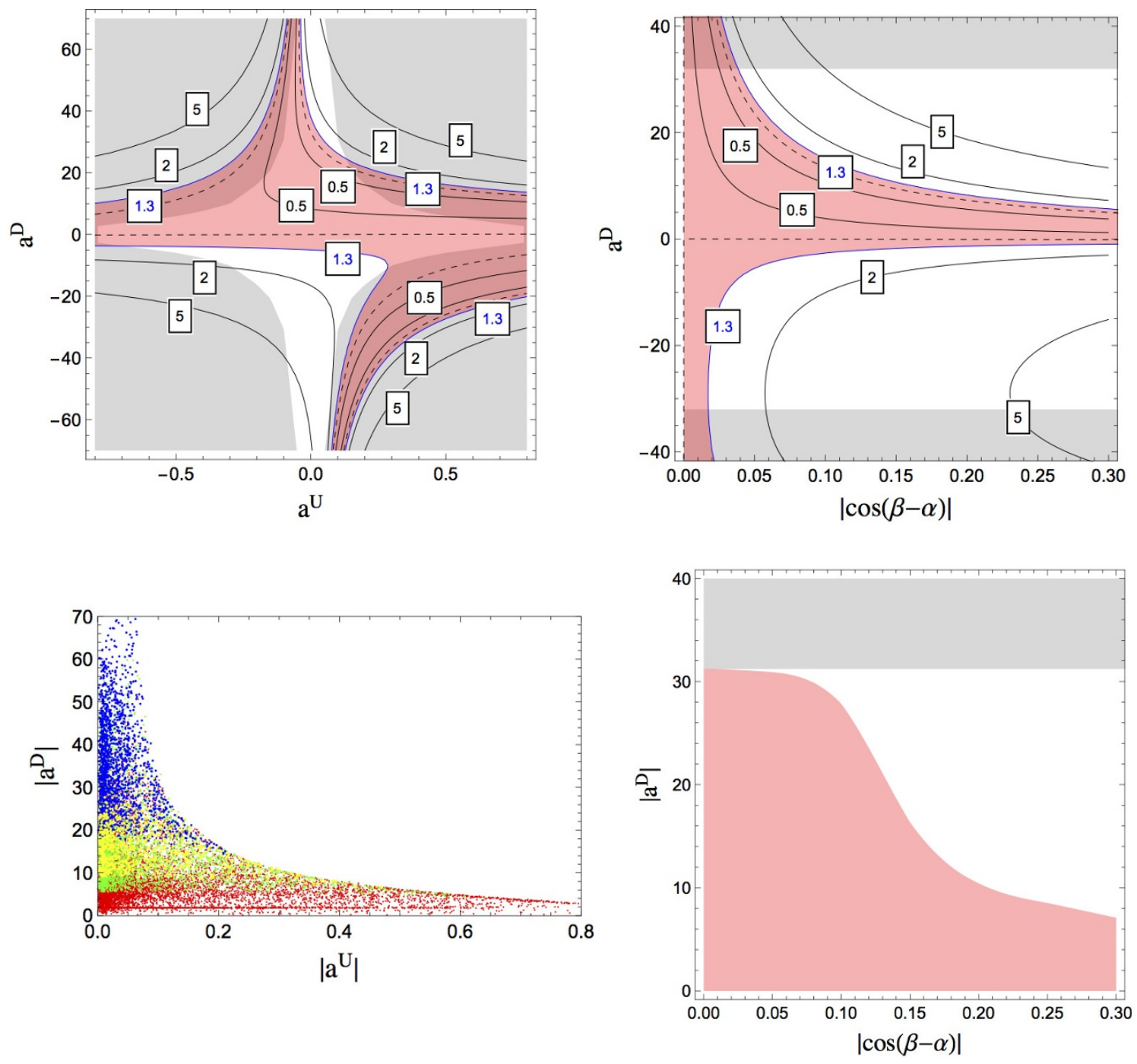

Figure 2. Prediction for the SM-like Higgs coupling to bottom quarks, normalized to the SM prediction, $\kappa_{b}$, as a function of $a^{U}$ and $a^{D}$, having fixed $|\cos (\beta-\alpha)|=0.05$ (left panels) and as a function of $|\cos (\beta-\alpha)|$ and $a^{D}$, having fixed $a^{U}=0.1$ (right panels). The top panels exhibit the leading $\log$ predictions. The dotted line corresponds to the SM value, $\kappa_{b}=1$. The gray shaded regions produce Landau poles in the Yukawa couplings below $M_{\mathrm{P}}$. The pink shaded region is favored by the LHC measurements of $\kappa_{b}$. The bottom panels show the corresponding results obtained via scanning the parameter space and using the full RG running. In the bottom left panel, yellow, red, green and blue colors correspond to values of $\kappa_{b}$ in the ranges $<0.5,[0.5, \mathbf{1 . 3}],[1.3,2]$ and $>2$, respectively. Here, the boldfaced number represents the $3 \sigma$ experimental upper bound of $\kappa_{b}$. All points shown correspond to parameter regimes where Landau poles are absent [cf. figure 1]. In the bottom right panel, the gray shaded region produces Landau poles in the Yukawa couplings below $M_{\mathrm{P}}$; the pink shaded region contains points favored by the LHC measurements of $\kappa_{b}$. 


\section{$3.2 \quad$ Flavor-changing top decays}

We calculate the branching ratios for the decays $t \rightarrow u_{i} h\left(u_{i}=u, c\right)$ arising from misalignment generated via radiative corrections during RG running. This is in contrast to the analysis of ref. [64] where flavor alignment is assumed to hold at the electroweak scale, in which case only charged Higgs loop diagrams contribute to the top flavor changing decays, leading to a $\mathrm{BR}\left(t \rightarrow u_{i} h\right)$ that depends strongly on the value of the charged Higgs mass. In this subsection, we show how the charged Higgs contributions compare to the those arising in our model due to tree-level flavor changing top couplings.

Following ref. [65], we employ the leading order formulae for both $t \rightarrow W b$ and $t \rightarrow u_{i} h$ decay rates, assuming the top quark decay width is dominated by the SM value of $\Gamma(t \rightarrow$ $W b)$. In addition, we include the NLO QCD correction to the branching ratio,

$$
\operatorname{BR}\left(t \rightarrow u_{i} h\right)=\cos ^{2}(\beta-\alpha)\left(\left|\rho_{i 3}^{U}\right|^{2}+\left|\rho_{3 i}^{U}\right|^{2}\right) \frac{v^{2}}{4 m_{t}^{2}} \frac{\left(1-m_{h}^{2} / m_{t}^{2}\right)^{2}}{\left(1-m_{W}^{2} / m_{t}^{2}\right)^{2}\left(1+2 m_{W}^{2} / m_{t}^{2}\right)} \eta_{Q C D}
$$

where $\eta_{Q C D}=1+0.97 \alpha_{s} \sim 1.10$. The flavor violating branching ratios scale with the second power of $\cos (\beta-\alpha)$, and thus suppressed in the $\cos (\beta-\alpha)=0$ limit. The couplings $\rho_{i 3}^{U}$ and $\rho_{3 i}^{U}$ can be easily extracted in the leading logarithmic approximation from eq. (2.74). Generically, the decay into a charm and a Higgs boson has a $\mathcal{O}\left(10^{2}\right)$ larger branching ratio than the decay into an up quark and a Higgs boson since in the leading logarithmic approximation,

$$
\frac{\mathrm{BR}(t \rightarrow c h)}{\mathrm{BR}(t \rightarrow u h)}=\frac{\left|\rho_{23}^{U}\right|^{2}+\left|\rho_{32}^{U}\right|^{2}}{\left|\rho_{13}^{U}\right|^{2}+\left|\rho_{31}^{U}\right|^{2}} \sim \frac{\left|\rho_{23}^{U}\right|^{2}}{\left|\rho_{13}^{U}\right|^{2}} \sim\left|\frac{K_{c b}}{K_{u b}}\right|^{2} .
$$

In the top panel of figure 3, we show the leading log results for the branching ratios, as a function of the two alignment parameters at the high scale, $a^{U}$ and $a^{D}$, having fixed $|\cos (\beta-\alpha)|=0.2$. Gray and pink shaded regions correspond to the region producing Landau poles in the Yukawa couplings below the Planck scale $M_{\mathrm{P}}$, and to the region favored by the LHC measurements of $\kappa_{b}$, respectively. Branching ratios larger than $\sim 10^{-10}$ for ch and $\sim 10^{-12}$ for $u h$ cannot be reached, while being consistent with Higgs coupling measurements and with the requirement of no Landau poles below $M_{\mathrm{P}}$. For comparison, we also show our results obtained scanning the parameter space and using the full RGEs (see the bottom panels of figure 3). Comparing the upper and lower panels of figure 3, we note that the agreement between the prediction at leading log and the full numerical results is less accurate at larger values of the alignment parameters $\left|a^{U}\right|$ and $\left|a^{D}\right|$, as expected. Values of the branching ratios as large as $\sim 3 \times 10^{-7}\left(\sim 3 \times 10^{-9}\right)$ for $t \rightarrow c h$ $(t \rightarrow u h)$ can be reached, while satisfying the condition due to the absence of Landau poles (see the blue points). However, the majority of points with $\operatorname{BR}(t \rightarrow c h) \gtrsim 10^{-8}$ and $\mathrm{BR}(t \rightarrow u h) \gtrsim 10^{-10}$ also produces too large a deviation from SM behavior of the Higgs coupling to bottom quarks. We have checked that the largest branching ratio compatible with Higgs data is at around $10^{-7}$ for $t \rightarrow c h$ and at around $10^{-9}$ for $t \rightarrow u h$.

These numbers should be compared with the corresponding contributions to flavorchanging top decays from charged Higgs loop diagrams, which are present in all 2HDMs, and are generated by flavor-changing charged Higgs interactions induced by CKM mixing $[64,66]$. Based on the discussion of ref. [64], we see that in the case of light charged 

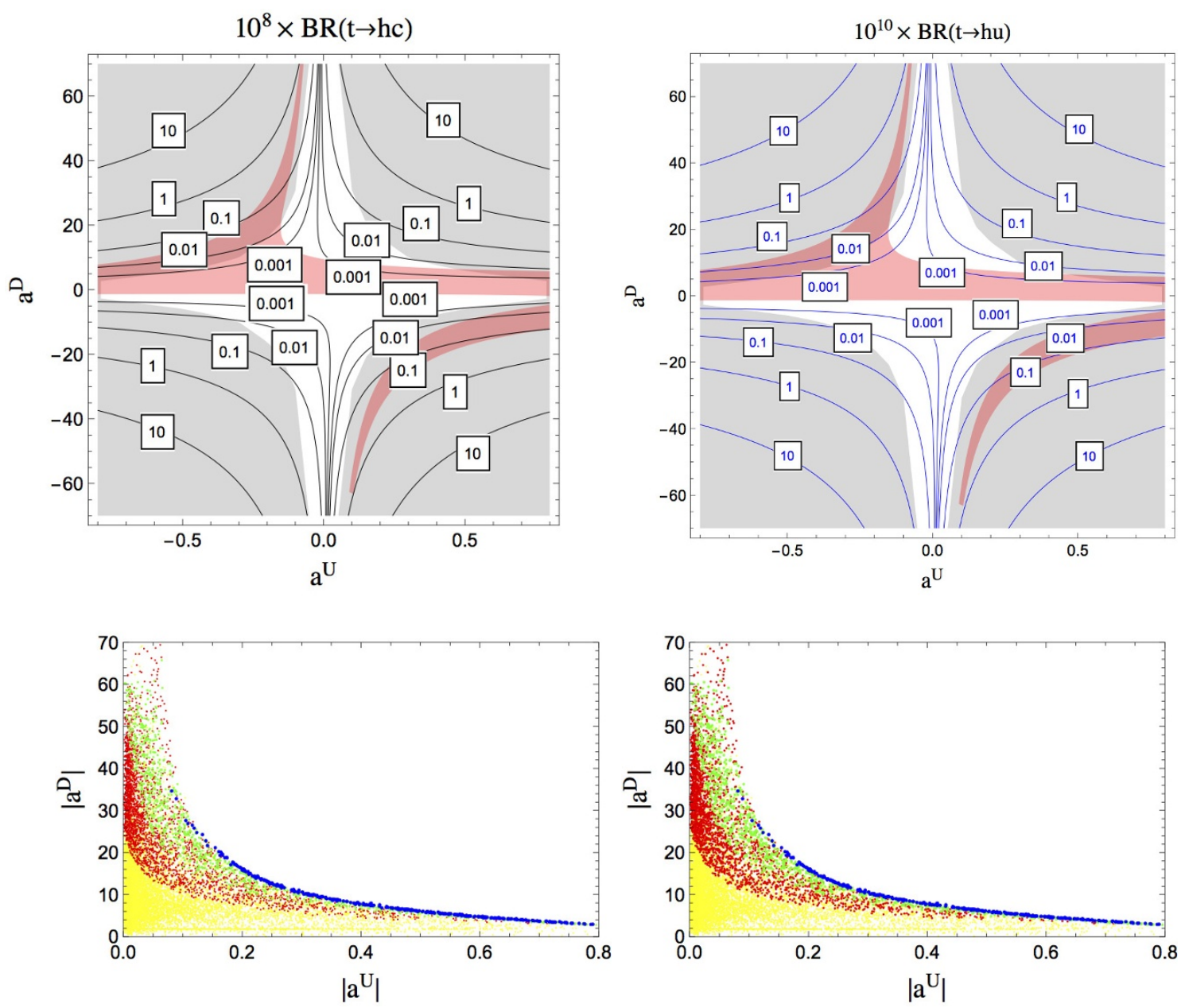

Figure 3. Tree-level contributions to top flavor changing decays assuming that $|\cos (\beta-\alpha)|=0.2$. Top panels: we use the leading $\log$ approximation to obtain $10^{8} \times \mathrm{BR}(t \rightarrow c h)$ [left panel] and $10^{10} \times$ $\mathrm{BR}(t \rightarrow u h)$ [right panel]. The gray shaded region produces Landau poles in the Yukawa couplings below $M_{\mathrm{P}}$. The pink region is favored by the LHC measurements of $\kappa_{b}$ (see section 3.1). Bottom panels: we exhibit the corresponding results obtained via the full RG running. Yellow, red, green and blue colors correspond to branching ratios $<10^{-11},\left[10^{-11}-10^{-10}\right],\left[10^{-10}-10^{-8}\right],>10^{-8}$ for $t \rightarrow c h$ [left panel], and to branching ratios $<10^{-13},\left[10^{-13}-10^{-12}\right],\left[10^{-12}-10^{-10}\right],>10^{-10}$ for $t \rightarrow h u$ [right panel].

Higgs bosons $\left(m_{H^{ \pm}} \lesssim 200 \mathrm{GeV}\right)$ and $h H^{+} H^{-}$couplings as large as allowed by $h \gamma \gamma$ constraints, these latter contributions can be as large as $\mathcal{O}\left(10^{-8}\right)$ and therefore comparable to those arising from the tree-level $h \bar{t} u_{i}$ coupling induced by RG-running in the A2HDM.

When compared to the $\mathrm{BR}(t \rightarrow c h)_{\mathrm{SM}} \sim 3 \times 10^{-15}, \mathrm{BR}(t \rightarrow u h)_{\mathrm{SM}} \sim 2 \times 10^{-17}$, as calculated in the SM by refs. [67-70], the $2 \mathrm{HDM}$ in general and the A2HDM in particular exhibit the possibility of a significant enhancement of the branching ratios for flavorchanging $t \rightarrow u_{i} h$ decays. However, both tree-level flavor changing effects and loop-level effects mediated by the charged Higgs boson are generically too small to be probed by the LHC and future colliders. 
Searches for top flavor changing decays have been performed by the ATLAS and CMS collaboration using Run I data [71-73], and constrain the branching ratios to $\mathrm{BR}(t \rightarrow$ $\left.u_{i} h\right) \lesssim 0.42 \%$ (see also [74] for a discussion of the most recent experimental results on top flavor changing decays). Projections for the HL-LHC show that the bounds on the branching ratios will likely be at the $10^{-4}$ level $[75,76]$. Hence, it will be very challenging to probe our model at the LHC using top flavor changing decays. FCC estimations show that branching ratios as small as $\sim 10^{-7}$ could be probed with $10 \mathrm{ab}^{-1}$ luminosity [77]. From these numbers, we can conclude that Higgs coupling measurements typically give (and will give) a better probe of our model, since the region of parameter space predicting more sizable top flavor violating branching ratios, also predict large and measurable effects in the Higgs coupling to bottom quarks.

\subsection{Phenomenology of the heavy Higgs bosons}

As pointed out in ref. [78], the $2 \mathrm{HDM}$ with flavor alignment imposed at the electroweak scale predicts a rich and novel phenomenology for the heavy Higgs bosons that is strikingly different than that of the 2HDM with Type I, II, X or Y Higgs-fermion Yukawa couplings. The phenomenology is even more diverse if flavor alignment is imposed at the high scale. For example, the heavy Higgs decay to quarks is flavor non-universal (i.e., the ratios, $y_{H d_{i} d_{i}} / m_{d_{i}}$ and $y_{H u_{i} u_{i}} / m_{u_{i}}$ are no longer independent of the flavor $i$ ). Moreover, flavor changing heavy Higgs decays, which are generated at the loop-level due to the quark flavor-changing charged Higgs interactions [63, 66], receive an additional contribution from tree-level flavor-changing neutral Higgs interactions. In contrast to the flavor-changing top decays discussed in the previous section, these features are not suppressed in the limit of $\cos (\beta-\alpha)=0$, where the couplings of $h$ coincide with those of the SM Higgs boson. This is exhibited by the tree-level partial widths of the heavy Higgs bosons to up and down quarks, which are given by

$$
\begin{aligned}
\Gamma\left(H \rightarrow \bar{f}_{i} f_{i}\right)= & \frac{3 G_{F}}{4 \sqrt{2} \pi} m_{H} m_{f_{i}}^{2}\left[\operatorname{Re}\left(c_{\beta-\alpha}-\epsilon_{6} s_{\beta-\alpha} \frac{\rho_{f}^{i i}}{\kappa_{f}^{i i}}\right)^{2}\left(1-\frac{4 m_{f_{i}}^{2}}{m_{H}^{2}}\right)^{3 / 2}\right. \\
& \left.+\operatorname{Im}\left(c_{\beta-\alpha}-\epsilon_{6} s_{\beta-\alpha} \frac{\rho_{f}^{i i}}{\kappa_{f}^{i i}}\right)^{2}\left(1-\frac{4 m_{f_{i}}^{2}}{m_{H}^{2}}\right)^{1 / 2}\right], \\
\Gamma\left(H \rightarrow \bar{f}_{i} f_{j}\right)= & \Gamma\left(H \rightarrow \bar{f}_{j} f_{i}\right)=\frac{3 G_{F}}{8 \sqrt{2} \pi} m_{H} v^{2} \times s_{\alpha-\beta}^{2}\left(\left|\rho_{f}^{i j}\right|^{2}+\left|\rho_{f}^{j i}\right|^{2}\right) \\
& \times\left[1-\left(\frac{m_{f_{i}}-m_{f_{j}}}{m_{H}}\right)^{2}\right]\left[\left(1-\frac{m_{f_{i}}^{2}+m_{f_{j}}^{2}}{m_{H}^{2}}\right)^{2}-\frac{4 m_{f_{i}}^{2} m_{f_{j}}^{2}}{m_{H}^{4}}\right]^{1 / 2}(i \neq j) .
\end{aligned}
$$

Henceforth, we shall set $\cos (\beta-\alpha)=0$, which automatically avoids constraints from the measured Higgs boson couplings. In the leading log approximation with real values of $a^{D}$ and $a^{U}$ assumed, the second term of eq. (3.5) can be neglected since $\operatorname{Im}\left(\rho_{f}^{i i}\right)=0$ [cf. eqs. (2.74) and (2.75)].

In figure 4, we show the leading log predictions for the most interesting branching ratios $\left(\bar{b} b, \bar{t} t, \tau^{+} \tau^{-}, \bar{b} s+\bar{s} b\right)$ as a function of the two alignment parameters $a^{U}$ and $a^{D}$, 

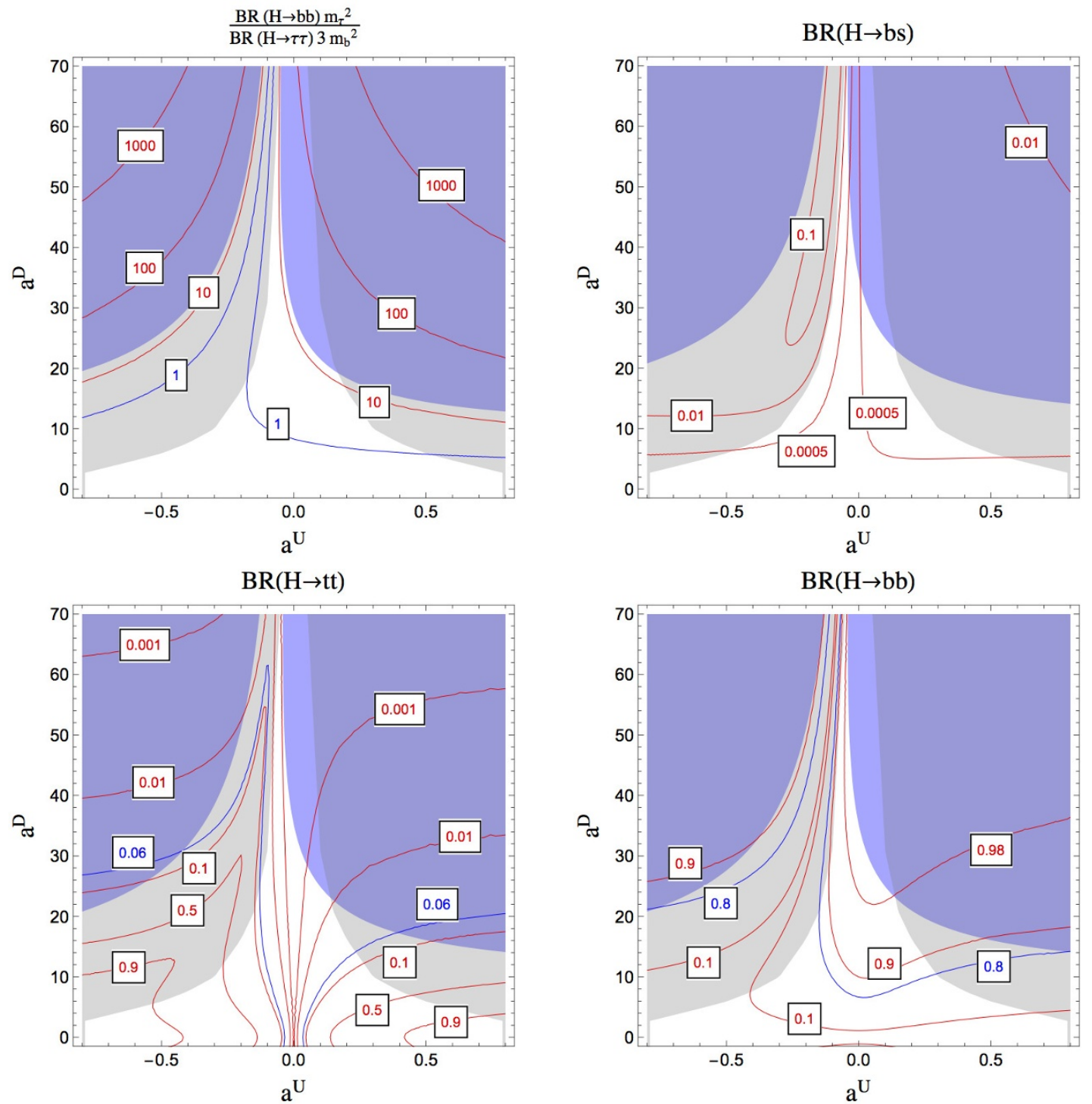

Figure 4. Leading log prediction for the branching ratios of the heavy Higgs boson, $H$, with fixed $\tan \beta=10, \cos (\beta-\alpha)=0$, and $m_{H}=400 \mathrm{GeV}$. The blue contours in the upper left and lower panels represent the prediction of a Type II 2 HDM. The gray shaded regions produce Landau poles below the Planck scale $\Lambda=M_{\mathrm{P}}$. The blue shaded regions have already been probed by the LHC searches for heavy scalars.

where we have fixed $\tan \beta=10$ and $m_{H}=400 \mathrm{GeV}$. In the two panels, we only show positive values of $a^{D}$, since the results are symmetric under $\left(a^{D}, a^{U}\right) \leftrightarrow\left(-a^{D},-a^{U}\right)$. For the predictions of $\operatorname{BR}(H \rightarrow \bar{b} s+\bar{s} b)$, we do not include loop contributions involving the charged Higgs boson. These latter contributions have been examined in refs. [63, 66] and have been shown generically to be considerably smaller than the corresponding treelevel flavor violating Higgs couplings. The left upper panel shows that in our model, especially at sizable values of the alignment parameters, the Type I and II 2HDM relation, $\mathrm{BR}(H \rightarrow \bar{b} b) / \mathrm{BR}\left(H \rightarrow \tau^{+} \tau^{-}\right)=3 m_{b}^{2} / m_{\tau}^{2}$, is violated. In particular, our model typically 

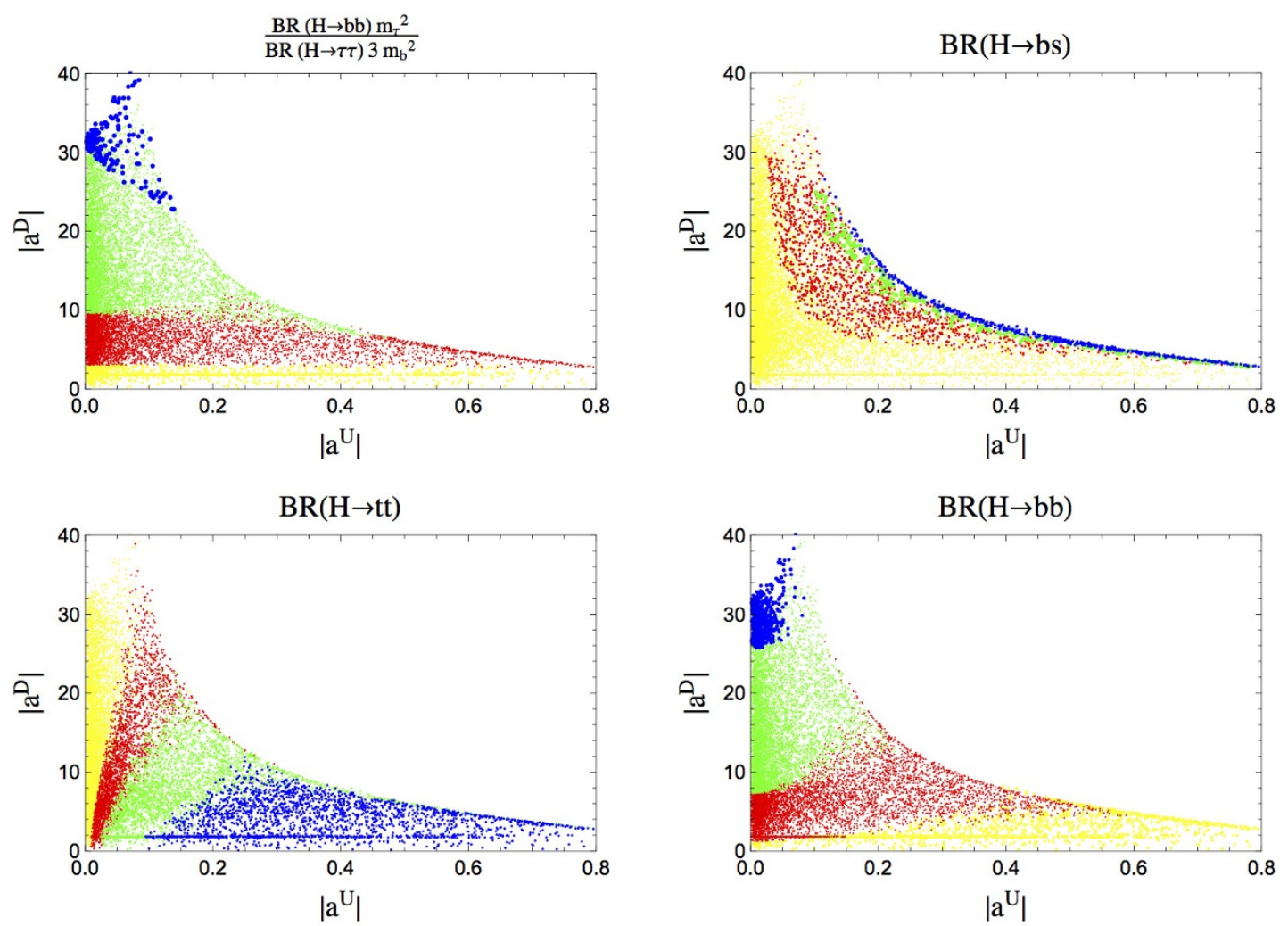

Figure 5. Branching ratios of the heavy Higgs boson, $H$, obtained by scanning the parameter space and using the full $\mathrm{RG}$ running, with fixed $\cos (\beta-\alpha)=0, \tan \beta=10$, and $m_{H}=400 \mathrm{GeV}$. The yellow, red, green and blue points correspond to: upper left panel, $\operatorname{BR}(H \rightarrow \bar{b} b) m_{\tau}^{2} / \operatorname{BR}\left(H \rightarrow \tau^{+} \tau^{-}\right) 3 m_{b}^{2}<\mathbf{1},[\mathbf{1}, 10],[10,100],>100 ;$ upper right panel, $\mathrm{BR}(H \rightarrow \bar{b} s+b \bar{s})<0.0005,[0.0005,0.01],[0.01,0.1],>0.1 ;$ lower left panel, $\mathrm{BR}(H \rightarrow \bar{t} t)<$ 0.01, $[0.01, \mathbf{0 . 0 6}],[\mathbf{0 . 0 6}, 0.5],>0.5$; and lower right panel, $\operatorname{BR}(H \rightarrow \bar{b} b)<0.1,[0.1, \mathbf{0 . 8}],[\mathbf{0 . 8}, 0.98],>$ 0.98. In boldface we denote the value of the branching ratios predicted by a Type II $2 \mathrm{HDM}$ with fixed $\tan \beta=10$. The parameter regime with $\left|a_{D}\right| \gtrsim 30-40$ and $\left|a_{U}\right| \lesssim 0.1$ has been eliminated after taking into account the LHC search for heavy Higgs bosons decaying into $\bar{b} b$ [79].

predicts a smaller ratio at small values of $a^{D}$, and therefore the $\tau^{+} \tau^{-}$mode is expected to be even more sensitive than $b \bar{b}$ relative to that of the Type I or II 2HDM. For $a^{D} \gtrsim 5$, the hierarchy is reversed, resulting in a larger $\operatorname{BR}(H \rightarrow \bar{b} b)$ as compared to $\operatorname{BR}\left(H \rightarrow \tau^{+} \tau^{-}\right)$. Furthermore, the model can predict a non zero decay rate of the heavy Higgs to a bottom and a strange quark (see the right upper panel of figure 4). However, the branching ratio predicted in the leading log approximation is at most of order a few percent at large values of $a^{D}$ in the regions of the parameter space without Landau poles.

Note that the branching ratios into third generation quarks are different as compared to the Type II 2HDM. In the latter, $\operatorname{BR}(H \rightarrow b \bar{b}) \sim 80 \%$ and $\operatorname{BR}(H \rightarrow t \bar{t}) \sim 6 \%$, for $\tan \beta=10$. For comparison, we present the branching ratios into $t \bar{t}$ and $b \bar{b}$ in the lower left and lower right panels of figure 4 . The behavior of the two plots is similar: at small values of $a^{U}\left(a^{D}\right)$ the $t \bar{t}(b \bar{b})$ branching ratio is smaller than the one predicted by the Type II 2HDM (see the blue contours in the two plots); the branching ratio can even vanish for particular 
choices of the alignment parameters $a^{U}$ and $a^{D}$. Larger values of the branching ratio are predicted for sizable values $a^{U} \gtrsim 0.035\left(a^{D} \gtrsim 7\right)$. As a byproduct, the ratio of branching ratios $\mathrm{BR}(H \rightarrow b \bar{b}) / \mathrm{BR}(H \rightarrow t \bar{t})$ differs from the predicted value of the $2 \mathrm{HDM}$ with either Type I, II, III, or IV Yukawa couplings. In particular, the A2HDM generically breaks the relation $\mathrm{BR}(H \rightarrow b \bar{b}) / \mathrm{BR}(H \rightarrow t \bar{t}) \simeq m_{b}^{2} \tan ^{4} \beta / m_{t}^{2}$, which is valid in the Type II $2 \mathrm{HDM}$ in the limit $\cos (\beta-\alpha)=0$. The branching ratios of a Type II $2 \mathrm{HDM}$ are recovered by choosing $a^{U}= \pm 1 / \tan \beta= \pm 1 / 10$ and $a^{D}=\mp \tan \beta=\mp 10$, as discussed at the end of section 2.3.

The ATLAS and CMS collaborations have performed several searches for heavy Higgs bosons decaying into a fermion pair: $\bar{b} b$ [79], $\tau^{+} \tau^{-}$[80, 81], $\mu^{+} \mu^{-}$[82, 83], and $\bar{t} t$ [84]. In a Type II $2 \mathrm{HDM}, \tau^{+} \tau^{-}$searches are the most important ones in constraining regions of parameter space at sizable values of $\tan \beta$. Searches for $\bar{b} b$ can only set weaker bounds in that scenario. However, as discussed e.g. in ref. [85], 2HDMs with a Yukawa texture different from Type II can be best probed by $\bar{b} b$ searches. In fact, for $\tan \beta=10$ and $\cos (\beta-\alpha)=0$, only the CMS search for $p p \rightarrow b(b) H, H \rightarrow \bar{b} b$, performed with $8 \mathrm{TeV}$ data [79], can probe sizable regions of the parameter space of the A2HDM (see the blue shaded region in figure 4 at large values of $a^{D}$ and the corresponding parameter regime of figure 5). In the coming years, the LHC will be able to probe complementary regions of parameter space. In addition to the region at large values of $a^{D}$ best probed by $\bar{b} b$ resonance searches, the region at small values of $a^{U}$ and $a^{D}$ will be best probed by searches for $\tau^{+} \tau^{-}$and $\mu^{+} \mu^{-}$resonances; and the region at small values of $a^{D}$, but sizable values of $a^{U}$ will be best probed by $\bar{t} t$ resonance searches.

For comparison, we show in figure 5 the corresponding results obtained through the scanning of the parameter space and the running of the full RGEs. Qualitatively, figure 5 shows a similar parameter dependence as the one obtained in the leading log approximation. Numerically, some branching ratios can be quite different, especially in the regime of sizable alignment parameters. In particular, $\operatorname{BR}(H \rightarrow \bar{b} s+b \bar{s})$ can reach values as large as $\sim 10 \%$.

\section{Predictions of the model for low energy processes}

As we discussed in section 2.7, the A2HDM is a particular type of $2 \mathrm{HDM}$ with Minimal Flavor Violation. As such, it predicts interesting effects in low energy flavor observables, e.g., in meson mixing and in $B$ meson rare decays. In this section, we shall discuss the predictions of our model for these low energy processes and the corresponding constraints. We shall focus on those observables that receive tree-level Higgs contributions, with particular attention to meson mixing, $B \rightarrow \mu^{+} \mu^{-}$, and $B \rightarrow \tau \nu$.

The lepton universality ratios, $\operatorname{BR}\left(\bar{B} \rightarrow D^{(*)} \tau^{-} \bar{\nu}\right) / \operatorname{BR}\left(\bar{B} \rightarrow D^{(*)} \ell^{-} \bar{\nu}\right)$, for $\ell=e, \mu$, are also notable, especially in light of the early BaBar measurements that yield a combined $3.4 \sigma$ deviation from the SM predictions [86, 87]. This anomaly is not inconsistent with subsequent Belle and $\mathrm{LHCb}$ measurements, even if with a smaller significance [88-91]. Additional data are required to clarify the implications of these measurements and to determine whether new physics beyond the SM is required. If this anomaly persists, New Physics models need (relatively large) $H^{ \pm} c_{L} b_{R}$ and $H^{ \pm} c_{R} b_{L}$ couplings of the same order and opposite sign (with $g_{H^{ \pm} c b}^{2} / m_{H^{ \pm}}^{2} \sim 1 / \mathrm{TeV}^{2}$ ), as shown in ref. [92]. This is rather 
challenging to achieve in our model while being consistent with the other flavor bounds. A more detailed examination of these channels will be left for a future study.

In principle, loop induced decays (which typically include contributions from the charged Higgs boson) can also set stringent constraints on the allowed regions of the $\left(m_{H^{ \pm}}, \tan \beta\right)$ parameter plane [93]. For example, in the Type II 2HDM the charged Higgs should be heavier than $580 \mathrm{GeV}$ [21] to be in agreement with $b \rightarrow s \gamma$ measurements (cf. footnote 2). Moreover, going beyond the Type II 2HDM, the $b \rightarrow s \gamma$ bound depends not only on the charged Higgs mass, but also on the values of $a^{U}$ and $a^{D}$, on other non-SM-like Higgs boson masses, as well as on potential contributions of New Physics particles in the loop. Such constraints merit further investigation. However, the analysis of this section focuses on parameter regimes in which tree-level Higgs-mediated FCNC effects dominate over competing one-loop contributions. For this reason, we do not consider further the constraints from $b \rightarrow s \gamma$ (which can be avoided for sufficiently heavy Higgs masses) in this paper.

\subsection{Meson mixing}

Higgs mediated contributions to neutral meson mixing $\left(B_{d, s}-\bar{B}_{d, s}, K-\bar{K}\right.$ and $D-\bar{D}$ mixing) arise in our model. Integrating out the three neutral Higgs bosons, we obtain the following dimension six effective Lagrangian describing $B_{s}$ meson mixing

$$
\mathscr{L}_{\text {eff }}=C_{2}\left(\bar{b}_{R} s_{L}\right)^{2}+\tilde{C}_{2}\left(\bar{b}_{L} s_{R}\right)^{2}+C_{4}\left(\bar{b}_{R} s_{L}\right)\left(\bar{b}_{L} s_{R}\right)+\text { h.c. }
$$

with Wilson coefficients,

$$
\begin{aligned}
& C_{2}=\frac{\left(\rho_{32}^{D}\right)^{2}}{4}\left(\frac{\sin ^{2}(\beta-\alpha)}{m_{H}^{2}}+\frac{\cos ^{2}(\beta-\alpha)}{m_{h}^{2}}-\frac{1}{m_{A}^{2}}\right), \\
& \tilde{C}_{2}=\frac{\left(\rho_{23}^{D *}\right)^{2}}{4}\left(\frac{\sin ^{2}(\beta-\alpha)}{m_{H}^{2}}+\frac{\cos ^{2}(\beta-\alpha)}{m_{h}^{2}}-\frac{1}{m_{A}^{2}}\right), \\
& C_{4}=\frac{\left(\rho_{32}^{D}\right)\left(\rho_{23}^{D *}\right)}{2}\left(\frac{\sin ^{2}(\beta-\alpha)}{m_{H}^{2}}+\frac{\cos ^{2}(\beta-\alpha)}{m_{h}^{2}}+\frac{1}{m_{A}^{2}}\right),
\end{aligned}
$$

and corresponding Wilson coefficients for $B_{d}, K$, and $D$ mixing.

In the case of degenerate heavy Higgs bosons and in the limit $\cos (\beta-\alpha)=0$, only $C_{4}$ contributes to meson mixing. In this limit, we expect small Wilson coefficients at leading $\log$, since as discussed in section 2.6, $\left|\left(\rho^{D}\right)_{i j}\right| \propto m_{i} / v$ and therefore $\left|\left(\rho^{D}\right)_{i j}\right| \ll\left|\left(\rho^{D}\right)_{j i}\right|$, for $i<j$. The Wilson coefficients are also relatively small away from the exact $\cos (\beta-\alpha)=0$ and $m_{A}=m_{H}$ limit. More specifically, $C_{2}$ and $\tilde{C}_{2}$ will be non zero, but suppressed by the combination of masses and mixing angles shown in eqs. (4.2) and (4.3), respectively. In the following, we will show the numerical results obtained for $\cos (\beta-\alpha)=0$ and $m_{A}=m_{H}=400 \mathrm{GeV}$. However, we have checked that the constraints on the parameter space do not change considerably by taking small but non-zero values for $\cos (\beta-\alpha)$.

We apply the bounds of ref. [94] on the $C_{4}$ Wilson coefficient (ref. [95] shows slightly stronger constraints). The leading $\log$ results for $B_{s}, B_{d}, K$, and $D$ mixing are shown in the left panel of figure 6 . The dark purple region is favored by the measurement of $B_{s}$ mixing, the purple region by $B_{d}$ mixing, the dark pink region by $\mathrm{CP}$ violation in the 

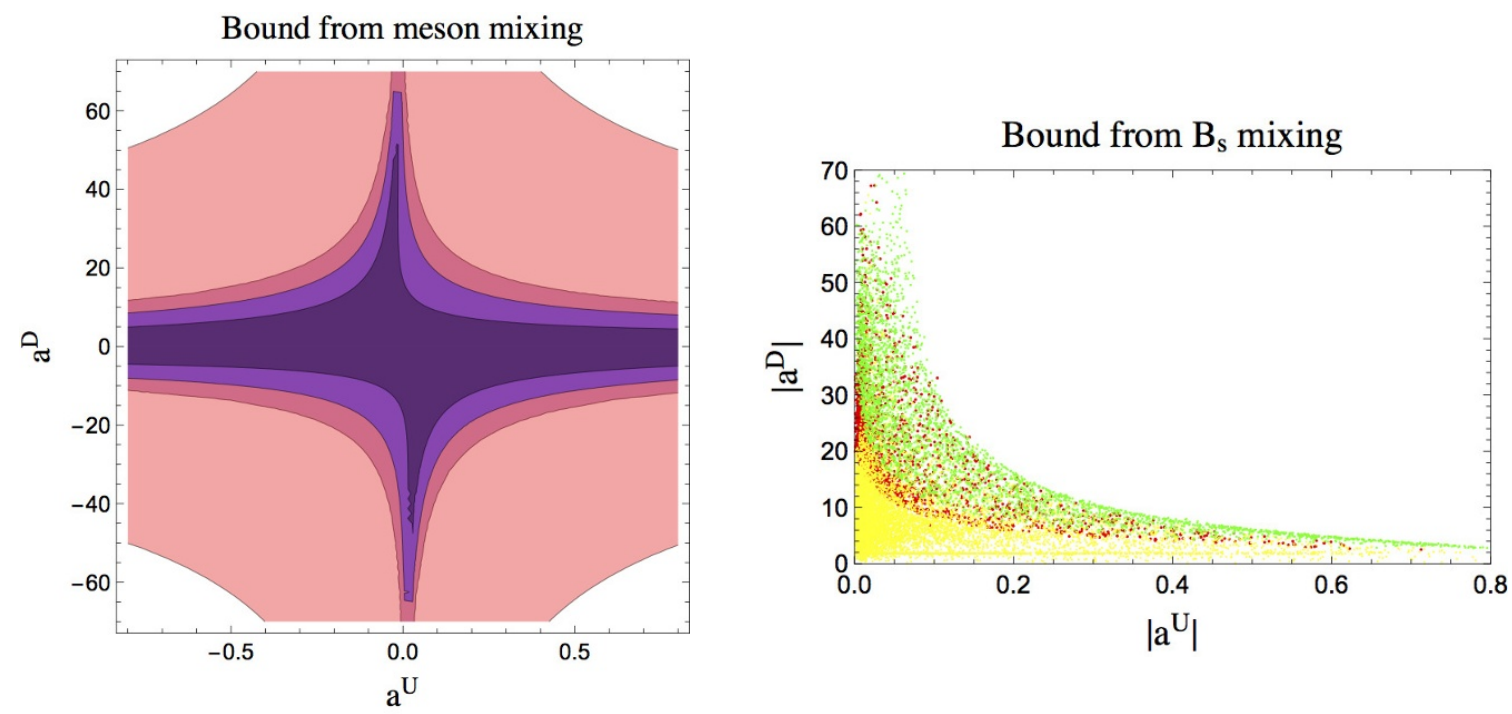

Figure 6. Bounds from meson mixing observables. Left panel: experimentally preferred regions, as computed in our model in the leading logarithmic approximation. The dark purple region is favored by the measurement of $B_{s}$ mixing, the purple region by $B_{d}$ mixing, and the dark pink (pink) region by the phase (mass difference) of the Kaon mixing system. $D$ meson mixing does not give any interesting bound on the parameter space and it is not shown in the figure. Right panel: the corresponding bounds from $B_{s}$ mixing obtained by scanning the parameter space and using the full RG running. The yellow, red, and green points correspond to a Wilson coefficient of $<1 / 3,[1 / 3, \mathbf{1}],>\mathbf{1}$ relative to the value that yields the present bound from $B_{s}$ mixing.

Kaon mixing system, and the pink region by the $K-\bar{K}$ mass difference. $D$ mixing does not give any interesting bound on the parameter space and is therefore omitted in the figure. $B_{s}$ mixing leads to the most stringent bound and it constrains $a^{D}$ to be smaller than $\sim 4.7$ at sizable values of $a^{U}$. Additionally, the bound from the measurement of CP violation in Kaon mixing (dark pink) is significantly more stringent than the bound from the mass difference of the Kaon system (in pink). This is due to the fact that the real and imaginary parts of the Wilson coefficient of the Kaon system have a similar magnitude (under the assumption that $a^{U}$ and $a^{D}$ are real). In particular, the ratio of the imaginary and real parts of the Wilson coefficient is directly related to the phase of the CKM matrix: $\operatorname{Im}\left(C_{4}^{K}\right) / \operatorname{Re}\left(C_{4}^{K}\right)=\operatorname{Im}\left(\left(K_{32}^{*}\right)^{2} K_{31}^{2}\right) / \operatorname{Re}\left(\left(K_{32}^{*}\right)^{2} K_{31}^{2}\right)$. In contrast, the SM Wilson coefficient has an imaginary part that is much smaller than the real part. Small differences between the constraints from $\mathrm{CP}$ violation and the mass difference also exist in the $B_{s}$ and $B_{d}$ systems. In figure 6 , we only show the most constraining bound in each system, i.e. the mass difference in $B_{s}$ mixing and the phase in $B_{d}$ mixing.

The right panel of figure 6 shows the corresponding results for the $B_{s}$ mixing system obtained by scanning the parameter space and using the full RG-running. The points in yellow have a Wilson coefficient smaller than $1 / 3$ the present bound on the Wilson coefficient; in red we present the points with a Wilson coefficient smaller than the present bound, and finally in green we present the points that have been already probed by the measurement of the $B_{s}$ mixing observables. In the limit of sizable $a^{U} \gtrsim 0.7$, we do not find points with $a^{D} \gtrsim 4$, in rough agreement with the leading log result. 


\section{2 $\quad B_{s, d} \rightarrow \mu^{+} \mu^{-}$decays}

The $B$-meson rare decays $B_{s, d} \rightarrow \ell^{+} \ell^{-}$receive contributions from the exchange of the Higgs bosons $H, A$ and $h$ at tree-level. This is in contrast to the numerical analysis of ref. [96], where the flavor misalignment at the electroweak scale is set to zero. The neutral Higgs exchange contributions to the leptonic decay amplitude are proportional to $m_{\ell}$ and hence are largest in the case of $B_{s, d} \rightarrow \tau^{+} \tau^{-}$. However, it is more difficult to tag the $\tau$ decay to jets and leptons at the LHC and B-factory detectors, as compared to muons. For this reason, the present LHCb bounds [97], $\mathrm{BR}\left(B_{s(d)} \rightarrow \tau^{+} \tau^{-}\right) \lesssim 3 \times 10^{-3}\left(1.3 \times 10^{-3}\right)$, are relatively weak as compared to the SM prediction [98],

$$
\operatorname{BR}\left(B_{s(d)} \rightarrow \tau^{+} \tau^{-}\right)_{\mathrm{SM}}=(7.73 \pm 0.49) \times 10^{-7}\left((2.22 \pm 0.19) \times 10^{-8}\right) .
$$

At sizable values of $\tan \beta$, the main contributions to $B_{s, d} \rightarrow \mu^{+} \mu^{-}$are typically due to $H$ and $A$ exchange, as they are enhanced by the second power of $\tan \beta$. Furthermore, in the $\cos (\beta-\alpha)=0$ limit, the light Higgs $(h)$ contribution vanishes at tree-level. For this reason, we shall focus henceforth on the heavy Higgs contributions that are given by [99],

$$
\frac{\operatorname{BR}\left(B_{s, d} \rightarrow \mu^{+} \mu^{-}\right)}{\operatorname{BR}\left(B_{s, d} \rightarrow \mu^{+} \mu^{-}\right)_{\mathrm{SM}}} \simeq\left(\left|S_{s, d}\right|^{2}+\left|P_{s, d}\right|^{2}\right)\left(1+y_{s, d} \frac{\operatorname{Re}\left(P_{s, d}^{2}\right)-\operatorname{Re}\left(S_{s, d}^{2}\right)}{\left|S_{s, d}\right|^{2}+\left|P_{s, d}\right|^{2}}\right)\left(\frac{1}{1+y_{s, d}}\right)
$$

where $\operatorname{BR}\left(B_{s, d} \rightarrow \mu^{+} \mu^{-}\right)_{\mathrm{SM}}$ is the $\mathrm{SM}$ prediction for the branching ratio extracted from an untagged rate. In particular, $y_{s}=(6.1 \pm 0.7) \%$ and $y_{d} \sim 0$ have to be taken into account when comparing experimental and theoretical results, and

$$
\begin{aligned}
S_{s, d} & \equiv \frac{m_{B_{s, d}}}{2 m_{\mu}} \frac{\left(C_{s, d}^{S}-C_{s, d}^{\prime S}\right)}{C_{10 s, d}^{S M}} \sqrt{1-\frac{4 m_{\mu}^{2}}{m_{B_{s, d}}^{2}}}, \\
P_{s, d} & \equiv \frac{m_{B_{s, d}}}{2 m_{\mu}} \frac{\left(C_{s, d}^{P}-C_{s, d}^{\prime P}\right)}{C_{10 s, d}^{S M}}+\frac{\left(C_{s, d}^{10}-C_{10 s, d}^{\prime}\right)}{C_{10 s, d}^{S M}} .
\end{aligned}
$$

The $C_{i}$ are the Wilson coefficients corresponding to the Lagrangian

$$
\mathscr{L}_{s}=\sum_{i}\left(C_{i} O_{i}+C_{i}^{\prime} O_{i}^{\prime}\right)+\text { h.c. },
$$

with operators

$$
\begin{aligned}
O_{s}^{(\prime) S} & =\frac{m_{b}}{m_{B_{s}}}\left(\bar{s} P_{R(L)} b\right)(\bar{\ell} \ell), \\
O_{s}^{(\prime) P} & =\frac{m_{b}}{m_{B_{s}}}\left(\bar{s} P_{R(L)} b\right)\left(\bar{\ell} \gamma^{5} \ell\right), \\
O_{10 s}^{(\prime)} & =\left(\bar{s} \gamma_{\mu} P_{L(R)} b\right)\left(\bar{\ell} \gamma^{\mu} \gamma^{5} \ell\right),
\end{aligned}
$$

and the corresponding ones for the $B_{d}$ system. In the limit of $\cos (\beta-\alpha)=0$, the Wilson coefficients arising from heavy neutral Higgs exchange are given by

$$
\begin{array}{rlrl}
C_{s}^{P} & =-\frac{m_{B_{s}}}{m_{b}} \frac{\rho_{32}^{D *}}{\sqrt{2}} \frac{m_{\mu}}{v} \tan \beta \frac{1}{m_{A}^{2}}, & C_{s}^{S} & =-\frac{m_{B_{s}}}{m_{b}} \frac{\rho_{32}^{D *}}{\sqrt{2}} \frac{m_{\mu}}{v} \tan \beta \frac{1}{m_{H}^{2}}, \\
C_{s}^{\prime P}=\frac{m_{B_{s}}}{m_{b}} \frac{\rho_{23}^{D}}{\sqrt{2}} \frac{m_{\mu}}{v} \tan \beta \frac{1}{m_{A}^{2}} \ll C_{s}^{P}, & C_{s}^{\prime S}=-\frac{m_{B_{s}}}{m_{b}} \frac{\rho_{23}^{D}}{\sqrt{2}} \frac{m_{\mu}}{v} \tan \beta \frac{1}{m_{H}^{2}} \ll C_{s}^{S},
\end{array}
$$


and the analogous results for the $B_{d}$ system. There are no tree-level New Physics contributions to the $\mathcal{O}_{10}^{(\prime)}$ operators.

If $\cos (\beta-\alpha)$ is nonvanishing, then the scalar Wilson coefficients $C_{s}^{S}$ and $C_{s}^{\prime S}$ given in eqs. (4.13) and (4.14) due to $H$ exchange should be changed accordingly, $\tan \beta \rightarrow$ $\sin (\beta-\alpha) \tan \beta+\cos (\beta-\alpha)$ and $\rho^{D} \rightarrow \rho^{D} \sin (\beta-\alpha)$. Moreover, an additional set of contributions arise due to $h$ exchange; the corresponding contributions are obtained from $C_{s}^{S}$ and $C_{s}^{\prime S}$ given in eqs. (4.13) and (4.14) by making the following replacements, $\tan \beta \rightarrow$ $\sin (\beta-\alpha)-\cos (\beta-\alpha) \tan \beta, \rho^{D} \rightarrow-\rho^{D} \cos (\beta-\alpha)$ and $m_{H} \rightarrow m_{h}$.

The SM Wilson coefficient takes the form [100],

$$
C_{10 s, d}^{\mathrm{SM}}=-4.1 \frac{e^{2}}{16 \pi^{2}} \frac{4 G_{F}}{\sqrt{2}} K_{t b} K_{t(s, d)}^{*},
$$

and the predicted branching ratios are given by

$$
\begin{aligned}
& \mathrm{BR}\left(B_{s} \rightarrow \mu^{+} \mu^{-}\right)_{\mathrm{SM}}=(3.65 \pm 0.23) \times 10^{-9}, \\
& \operatorname{BR}\left(B_{d} \rightarrow \mu^{+} \mu^{-}\right)_{\mathrm{SM}}=(1.06 \pm 0.09) \times 10^{-10},
\end{aligned}
$$

as obtained in [98] with the inclusion of $\mathcal{O}\left(\alpha_{e m}\right)$ and $\mathcal{O}\left(\alpha_{s}^{2}\right)$ corrections. These values are in relatively good agreement with the experimental results. The combination of the $\mathrm{LHCb}$ and the CMS measurements at Run I for the $B_{s}$ and $B_{d}$ decays to muon pairs are [101]:

$$
\begin{aligned}
& \operatorname{BR}\left(B_{s} \rightarrow \mu^{+} \mu^{-}\right)=\left(2.8_{-0.6}^{+0.7}\right) \times 10^{-9}, \\
& \operatorname{BR}\left(B_{d} \rightarrow \mu^{+} \mu^{-}\right)=\left(3.9_{-1.4}^{+1.6}\right) \times 10^{-10} .
\end{aligned}
$$

Note the much larger uncertainty in the latter decay mode.

The ATLAS collaboration has also reported a Run I search for $B_{s} \rightarrow \mu^{+} \mu^{-}$, which yielded $\operatorname{BR}\left(B_{s} \rightarrow \mu^{+} \mu^{-}\right)=\left(0.9_{-0.8}^{+1.1}\right) \times 10^{-9}$ [102], although this measurement is not yet competitive with eq. (4.18). Very recently, LHCb reported a new measurement for $B_{s, d} \rightarrow$ $\mu^{+} \mu^{-}$using Run II data [103]. Their result, $\operatorname{BR}\left(B_{s} \rightarrow \mu^{+} \mu^{-}\right)=(2.8 \pm 0.6) \times 10^{-9}$, agrees very well with the $\mathrm{LHCb}$ and CMS combination quoted in eq. (4.18). In contrast, the new $\mathrm{LHCb} B_{d}$ measurement is closer to the SM prediction, $\mathrm{BR}\left(B_{d} \rightarrow \mu^{+} \mu^{-}\right)=\left(1.6_{-0.9}^{+1.1}\right) \times 10^{-10}$. In the following, we will compare the predictions of the A2HDM with the LHCb and CMS combination shown in eqs. (4.18) and (4.19). In the coming years, the two branching ratios will be measured much more accurately by the LHC. In particular, the $B_{s}$ and $B_{d}$ branching fractions will be measured by each experiment with a precision of $\sim 13 \%$ and $\sim 48 \%$ at Run-III, improving to $\sim 11 \%$ and $\sim 18 \%$, respectively, at the HL-LHC [104].

In figure 7 , we show the constraints from the measurement of $B_{s} \rightarrow \mu^{+} \mu^{-}$(left panel) and $B_{d} \rightarrow \mu^{+} \mu^{-}$(right panel) as functions of $a^{U}$ and $a^{D}$, with fixed $\tan \beta=10, \epsilon_{E}=+1$ [see eq. (2.59)], ${ }^{21} \cos (\beta-\alpha)=0$, and $m_{A}=m_{H}=400 \mathrm{GeV}$, based on the leading logarithmic approximation. The pink shaded region denote the parameter space favored by the CMS and LHCb combined results at the $2 \sigma$ level, namely

$$
\frac{\mathrm{BR}\left(B_{s} \rightarrow \mu^{+} \mu^{-}\right)}{\operatorname{BR}\left(B_{s} \rightarrow \mu^{+} \mu^{-}\right)_{S M}} \subset[0.4,1.1], \quad \frac{\mathrm{BR}\left(B_{d} \rightarrow \mu^{+} \mu^{-}\right)}{\operatorname{BR}\left(B_{d} \rightarrow \mu^{+} \mu^{-}\right)_{S M}} \subset[0.8,6.6] .
$$

\footnotetext{
${ }^{21}$ Fixing a different sign, $\epsilon_{E}=-1$, leads to the same results, with the exchange $\left(a^{U}, a^{D}\right) \rightarrow\left(-a^{U},-a^{D}\right)$.
} 

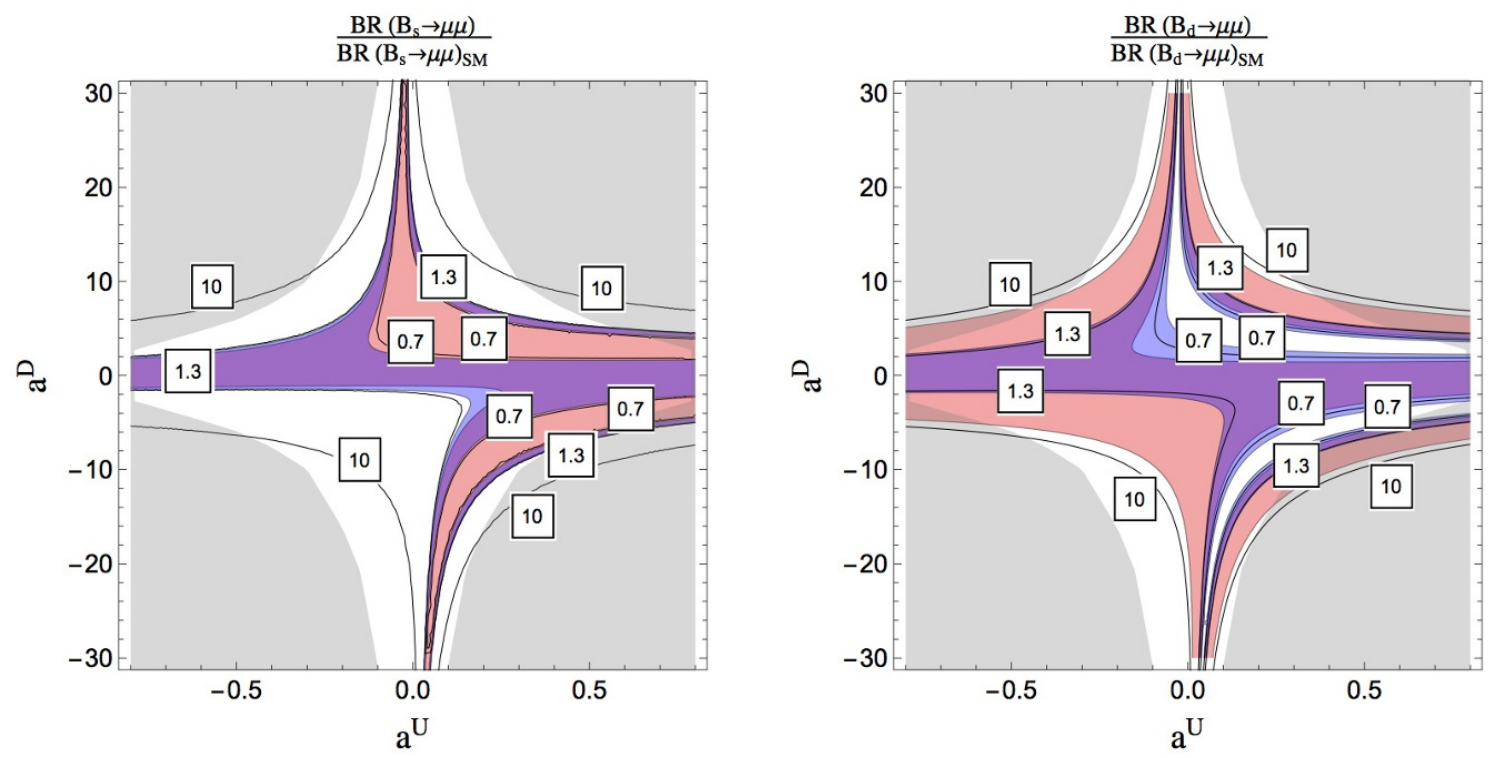

Figure 7. Leading log prediction for the branching ratios for $B_{s} \rightarrow \mu^{+} \mu^{-}$(left panel) and $B_{d} \rightarrow$ $\mu^{+} \mu^{-}$(right panel) relative to the SM, as a function of $a^{U}$ and $a^{D}$, with fixed $\tan \beta=10, \cos (\beta-$ $\alpha)=0$, and $m_{A}=m_{H}=400 \mathrm{GeV}$. The regions in pink are allowed at the $2 \sigma$ level by the present measurements. The purple shaded regions are anticipated by the more precise HL-LHC measurements, assuming a measured central value equal to the SM prediction. The gray shaded regions produce Landau poles in the Yukawa couplings below $M_{\mathrm{P}}$.

The purple shaded region in figure 7 is the parameter space favored at $2 \sigma$ by the HL-LHC measurement, assuming a measured central value equal to the SM prediction. Comparing the region in pink to the region in purple, one can get a sense of the improvement the HLLHC can achieve in testing our model. The expected experimental error at the HL-LHC is comparable to the present theory error. For this reason, an additional improvement can be achieved via a more precise calculation of the SM prediction for the two branching ratios, with the benefit of more accurate measurements of the CKM elements that will be obtained at the LHCb and at Belle II in the coming years.

The present measurement of $B_{s} \rightarrow \mu^{+} \mu^{-}$constrains sizable values of $a^{U}$ and $a^{D}$ in our model. The measurement of $B_{d} \rightarrow \mu^{+} \mu^{-}$also sets an interesting constraint at smaller values of $\left|a^{D}\right|$ (cf. the white region where $\left|a^{D}\right| \sim 3$ and the values of $\left|a^{U}\right|$ are sizable), since the central value of the measurement is larger than the $\mathrm{SM}$ prediction: $\operatorname{BR}\left(B_{d} \rightarrow\right.$ $\left.\mu^{+} \mu^{-}\right)_{\exp } / \operatorname{BR}\left(B_{d} \rightarrow \mu^{+} \mu^{-}\right)_{\mathrm{SM}} \sim 3.7$. However, the deviation from the SM prediction is not yet statistically significant, due to the large experimental uncertainty. Nevertheless, a sizable suppression of the $B_{d}$ decay mode is presently disfavored. As expected, the contours for $\operatorname{BR}\left(B_{s, d} \rightarrow \mu^{+} \mu^{-}\right) / \operatorname{BR}\left(B_{s, d} \rightarrow \mu^{+} \mu^{-}\right)_{\mathrm{SM}}$ in the two panels of figure 7 are very similar. This is due to the fact that our model is a particular type of MFV model in the leading logarithmic approximation [cf. section 2.7]. In particular, MFV models generically predict $\mathrm{BR}\left(B_{d} \rightarrow \mu^{+} \mu^{-}\right) / \mathrm{BR}\left(B_{s} \rightarrow \mu^{+} \mu^{-}\right) \sim \mathrm{BR}\left(B_{d} \rightarrow \mu^{+} \mu^{-}\right)_{\mathrm{SM}} / \mathrm{BR}\left(B_{s} \rightarrow \mu^{+} \mu^{-}\right)_{\mathrm{SM}}$, with corrections arising only from $m_{s} / m_{b}$ and $m_{d} / m_{b}$ terms. For this reason, it is difficult in our model to enhance one decay mode, while suppressing the other. 

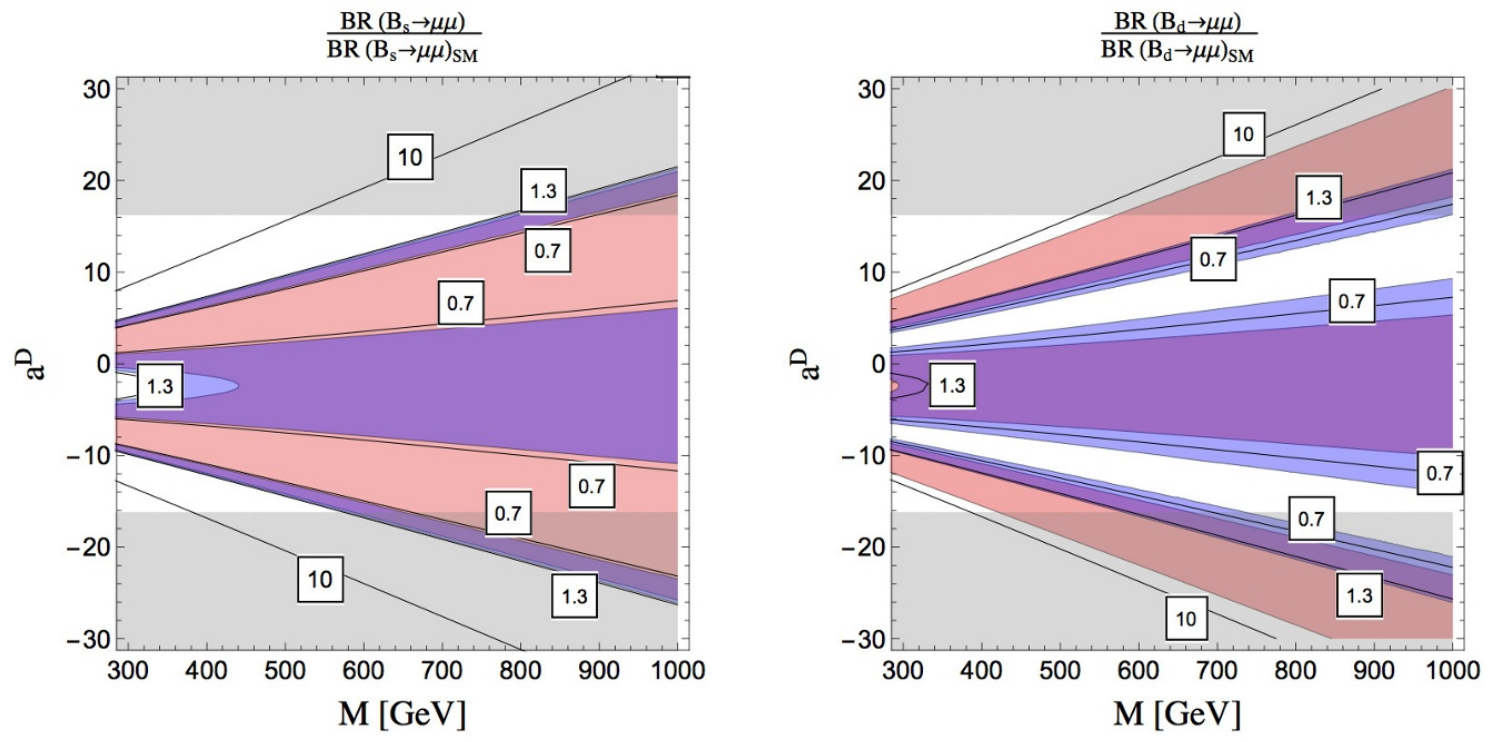

Figure 8. Leading log prediction for the branching ratios for $B_{s} \rightarrow \mu^{+} \mu^{-}$(left panel) and $B_{d} \rightarrow$ $\mu^{+} \mu^{-}$(right panel) relative to the SM, as a function of $M$ (the mass of the heavy scalar and pseudoscalar) and $a^{D}$. We fix $\tan \beta=10, a^{U}=0.2$, and $\cos (\beta-\alpha)=0$. The pink regions are the regions allowed at the $2 \sigma$ level by the present measurements. The purple regions are anticipated by the more precise HL-LHC measurements, assuming a measured central value equal to the SM prediction. The gray shaded regions produce Landau poles in the Yukawa couplings below $M_{\mathrm{P}}$.
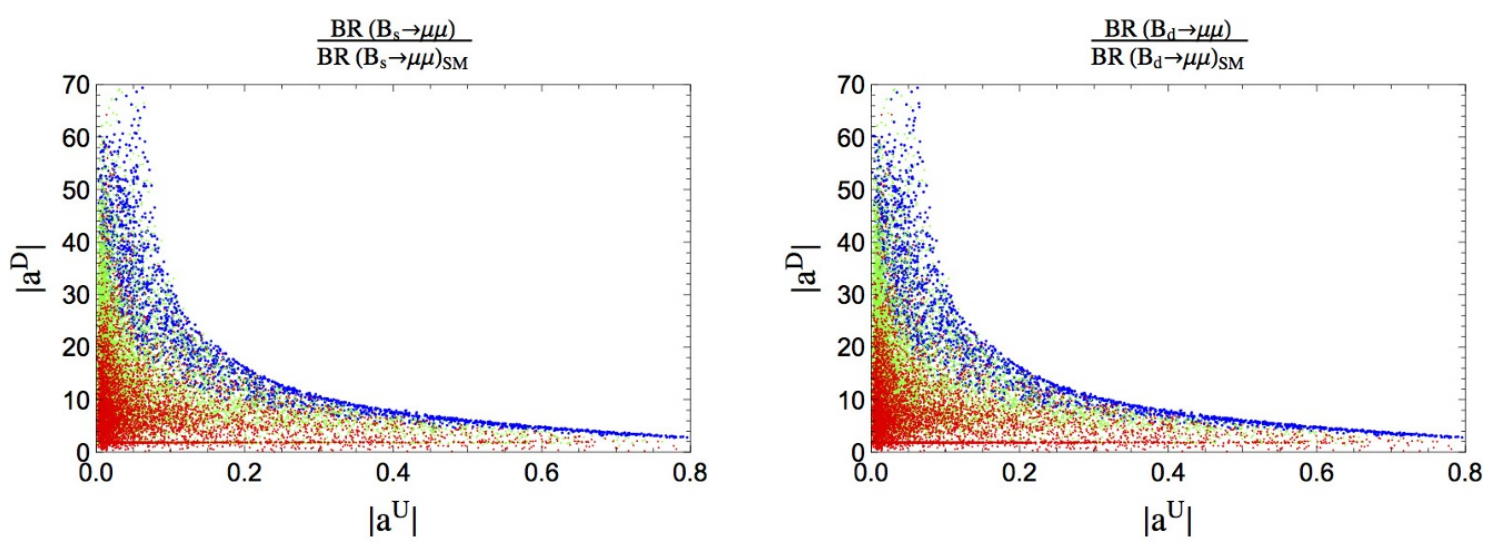

Figure 9. The branching ratio for $B_{s} \rightarrow \mu^{+} \mu^{-}$(left panel) and for $B_{d} \rightarrow \mu^{+} \mu^{-}$(right panel) relative to the SM, obtained via scanning the parameter space and using the full RG running, at fixed $\tan \beta=10, \cos (\beta-\alpha)=0$, and $m_{A}=m_{H}=400 \mathrm{GeV}$. The yellow, red, green and blue points corresponds to branching ratios normalized to the SM prediction $<\mathbf{0 . 4},[\mathbf{0 . 4}, \mathbf{1 . 1}],[\mathbf{1 . 1}, 10],>10$. In boldface we denote the range preferred by the LHCb and ATLAS measurement of $B_{s} \rightarrow \mu^{+} \mu^{-}$, as reported in eq. (4.20). 
It is also interesting to investigate the bounds as a function of the heavy Higgs boson masses. In figure 8 , we show the same constraints in the $\left(M, a^{D}\right)$ plane, where $M \equiv m_{A}=$ $m_{H}$, having fixed $\tan \beta=10, a^{U}=0.2$, and $\cos (\beta-\alpha)=0$. Sizable regions of parameter space are allowed, even for values of $M$ as small as $\sim 300 \mathrm{GeV}$. Finally, in figure 9 , we show the results obtained through scanning the parameter space and utilizing the full RG running. These plots are qualitatively similar to the contour plots of figure 7 obtained in the leading logarithmic approximation, although the heavy Higgs exchange contributions to the $B_{d, s} \rightarrow \mu^{+} \mu^{-}$decay rates computed using the full RG running are somewhat larger (at large alignment parameters) than the corresponding leading log results.

\section{3 $B \rightarrow \tau \nu$ decays}

The leptonic decays $B \rightarrow \ell \nu$ are interesting probes of the Higgs sector of our model and particularly of the charged Higgs couplings, since the charged Higgs boson mediates treelevel New Physics contributions to these decay modes. The $\tau$ channel is the only decay mode of this type observed so far. The present experimental world average is $[105]^{22}$

$$
\mathrm{BR}(B \rightarrow \tau \nu)_{\exp }=(1.06 \pm 0.19) \times 10^{-4},
$$

and is in relatively good agreement with the SM prediction $[106]^{23}$

$$
\mathrm{BR}(B \rightarrow \tau \nu)_{\mathrm{SM}}=\left(0.848_{-0.055}^{+0.036}\right) \times 10^{-4} .
$$

In our model, the New Physics contribution to this decay reads

$$
\frac{\mathrm{BR}(B \rightarrow \tau \nu)}{\mathrm{BR}(B \rightarrow \tau \nu)_{\mathrm{SM}}}=\left|1+\frac{m_{B}^{2}}{m_{b} m_{\tau}} \frac{C_{L}^{u b}-C_{R}^{u b}}{C_{\mathrm{SM}}^{u b}}\right|^{2},
$$

where we have defined the SM Wilson coefficient $C_{\mathrm{SM}}^{u b}=4 G_{F} K_{u b} / \sqrt{2}$ and $C_{R(L)}^{u b}$ are the Wilson coefficients of the $\mathcal{O}_{R(L)}^{u b}=\left(\bar{u} P_{R(L)} b\right)\left(\bar{\tau} P_{L} \nu_{\tau}\right)$ operators.

In particular [107],

$$
C_{R(L)}^{u b}=\frac{1}{m_{H^{ \pm}}^{2}} \Gamma_{u b}^{L R(R L)} \frac{\sqrt{2} m_{\tau}}{v} \tan \beta
$$

with $\Gamma_{u b}^{L R(R L)}$ the two charged Higgs couplings $H^{+} \bar{u}_{L} b_{R}, H^{+} \bar{u}_{R} b_{L}$ given by

$$
\Gamma_{u b}^{L R}=\sum_{i} K_{u i} \rho_{3 i}^{D *}, \quad \Gamma_{u b}^{R L}=-\sum_{i} K_{i b}^{*} \rho_{i 1}^{U *}
$$

This leads to the branching ratio,

$$
\frac{\mathrm{BR}(B \rightarrow \tau \nu)}{\mathrm{BR}(B \rightarrow \tau \nu)_{\mathrm{SM}}}=\left|1-\frac{m_{B}^{2}}{m_{b}} \frac{v \tan \beta}{\sqrt{2} K_{u b} m_{H^{ \pm}}^{2}} \sum_{i}\left[K_{u i} \rho_{3 i}^{D *}+K_{i b}^{*} \rho_{i 1}^{U *}\right]\right|^{2}
$$

\footnotetext{
${ }^{22}$ Updated results and plots available at: http://www.slac.stanford.edu/xorg/hfag.

${ }^{23}$ Updated results and plots available at: http://ckmfitter.in2p3.fr.
} 


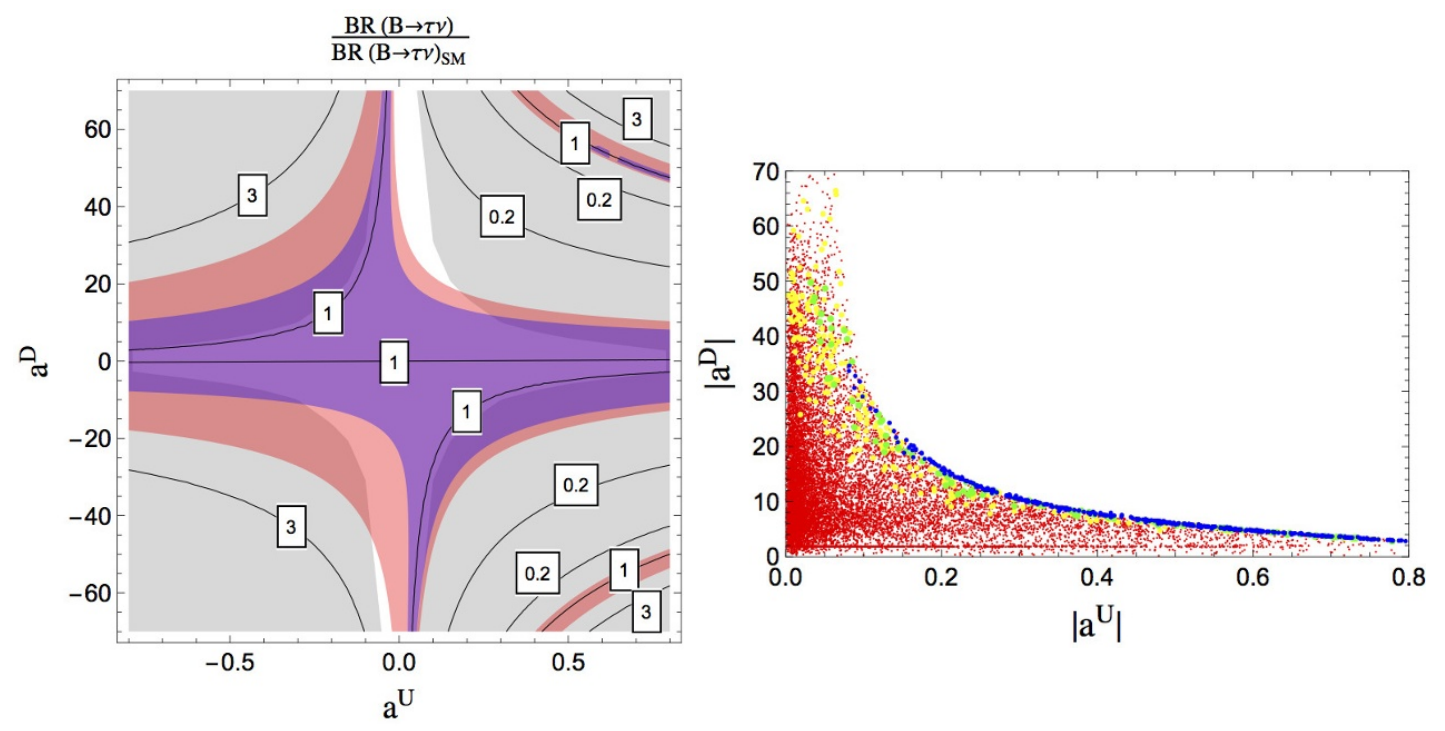

Figure 10. The ratio $\mathrm{BR}(B \rightarrow \tau \nu) / \mathrm{BR}(B \rightarrow \tau \nu)_{\mathrm{SM}}$ at fixed $\tan \beta=10$ and $m_{H^{ \pm}}=400 \mathrm{GeV}$. Left panel: leading log predictions, where the pink region is favored by the measurement of $B \rightarrow \tau \nu$. The purple region is anticipated by future measurement at Belle II, under the assumption that the central value of the measurement is given by the SM prediction. Right panel: result of the parameter space scan, using the full RG running. Yellow, red, green and blue points correspond to the ratios $<0.2,[\mathbf{0 . 7 9}, \mathbf{1 . 7 1}],[\mathbf{1 . 7 1}, 3],>3$, respectively. In boldface we denote the range preferred by the present world average for $\operatorname{BR}(B \rightarrow \tau \nu)$.

In the leading logarithmic approximation, the most important contributions come from the second term of the above expression $\left(\propto \rho_{3 i}^{D *}\right)$, as one can easily deduce from eqs. (2.74) and (2.75).

In figure 10, we show our numerical results as obtained using the leading log approximation (left panel) and the scan of the parameter space using the full RGEs, having fixed $m_{H^{ \pm}}=400 \mathrm{GeV}$ and $\tan \beta=10$. A very large region of parameter is still allowed by the measurement of $B \rightarrow \tau \nu$. In particular, in the leading logarithmic approximation, every value $\left|a^{D}\right| \lesssim 17$ is allowed, irrespective of the value of the other alignment parameter, $a^{U}$. Indeed, in the pink region shown in the left panel of figure 10, $\mathrm{BR}(B \rightarrow \tau \nu) / \mathrm{BR}(B \rightarrow \tau \nu)_{\mathrm{SM}} \subset[0.79,1.71]$, consistent with the current measurements. This is no longer the case when we consider the scan based on the full RG-running. In this case, a few points at large values of $\left|a^{U}\right|$ are excluded by the measurement of $\operatorname{BR}(B \rightarrow \tau \nu)$ (see the blue points in the right panel of the figure). In the left panel of figure 10, we also exhibit the purple shaded region of parameter space that would be favored by the future Belle II measurement, under the assumption that the central value of the measurement is given by the SM prediction for this branching ratio [cf. eq. (4.22)]. The allowed region of parameter space is expected to shrink considerably, thanks to the anticipated accuracy of the Belle II measurement with a total error of the order of $\sim 5 \%$ [108], leading to an allowed range, $\operatorname{BR}(B \rightarrow \tau \nu) / \mathrm{BR}(B \rightarrow \tau \nu)_{\mathrm{SM}} \subset[0.86,1.14]$, where we have assumed no improvement in the SM prediction of this $B$ meson decay mode. 


\section{Conclusions}

We have explored the consequences of flavor-alignment at a very high energy scale on flavor observables in the two Higgs doublet Model (2HDM). Flavor alignment at the electroweak scale generically requires an unnatural fine-tuning of the matrix Yukawa couplings. If flavor alignment is instead imposed at a higher energy scale such as the Planck scale, perhaps enforced by some new dynamics beyond the SM, then the flavor misalignment at the electroweak scale due to RG running will generate new sources of FCNCs. The resulting tree-level Higgs-mediated FCNCs are somewhat suppressed and relatively mildly constrained by experimental measurements of flavor-changing observables.

We require that the alignment parameters at the high scale remain perturbative. In particular, no Landau poles are encountered during RG running. These requirements lead to an upper bound on the values of the alignment parameters at the Planck scale. This in turn provide an upper bound on the size of FCNCs generated at the electroweak scale. The flavor-changing observables considered in this paper that provide the most sensitive probe of the flavor-aligned $2 \mathrm{HDM}$ parameter space are meson mixing and rare $B$ decays such as $B_{s, d} \rightarrow \mu^{+} \mu^{-}$and $B \rightarrow \tau \nu$. We also considered constraints from LHC searches of heavy Higgs bosons (the most important of which are searches for $p p \rightarrow b(b) H, H \rightarrow$ $\bar{b} b, \tau^{+} \tau^{-}$), and measurements of the couplings of the observed (SM-like) Higgs boson. The most stringent constraint on the flavor-aligned $2 \mathrm{HDM}$ parameter space arises from the measurement of the rare decay $B_{s} \rightarrow \mu^{+} \mu^{-}$.

We investigated the predictions of the flavor-aligned $2 \mathrm{HDM}$ in the regions of the parameter space not yet probed by the measurements listed above. The top rare flavor changing decays, $t \rightarrow u h, t \rightarrow c h$, are generated at tree-level. However, once we impose constraints from Higgs coupling measurements, the predicted branching ratios for these neutral flavor changing top decays are beyond the LHC reach. Furthermore, the model predicts a novel phenomenology for the heavy Higgs bosons. In particular, the heavy Higgs bosons can have a sizable branching ratios into a bottom and a strange quark, and the ratios, $\mathrm{BR}(H \rightarrow \bar{t} t): \mathrm{BR}(H \rightarrow \bar{b} b): \mathrm{BR}\left(H \rightarrow \tau^{+} \tau^{-}\right)$, can be very different, if compared to the predictions of the more common Type I and II 2HDMs. These features are exhibited in our summary plots in figures 11 and 12.

In figure 11, we summarize the constraints on the $\left(a^{U}, a^{D}\right)$ parameter space, with fixed $\tan \beta=10$ (upper panels) and $\tan \beta=3$ (lower panels). In both panels, we fix the values $\cos (\beta-\alpha)=0$ and $m_{A}=m_{H}=m_{H^{ \pm}}=400 \mathrm{GeV}$. The region favored by all flavor constraints is shown in reddish-brown. At sizable values of $a^{D}$, the most relevant constraint comes from the measurement of $B_{s} \rightarrow \mu^{+} \mu^{-}$(tan region). $B_{s}$ meson mixing also sets an interesting bound on the parameter space (blue-gray region). It offers some complementary with $B_{s} \rightarrow \mu^{+} \mu^{-}$, as it does not depend on the particular value of $\tan \beta$. Moreover, it will be able to probe the small region of parameter space with $a^{U}>0$ and sizable values of $a^{D}$ favored by the measurement of $B_{s} \rightarrow \mu^{+} \mu^{-}$in the case of a future measurement with a central value in agreement with the SM prediction. ${ }^{24}$ The measurement of $B \rightarrow \tau \nu$ imposes only a

\footnotetext{
${ }^{24}$ We use the results in [109] for the future prospects in measuring $B_{s}$ mixing, corresponding to the "Stage II" scenario.
} 

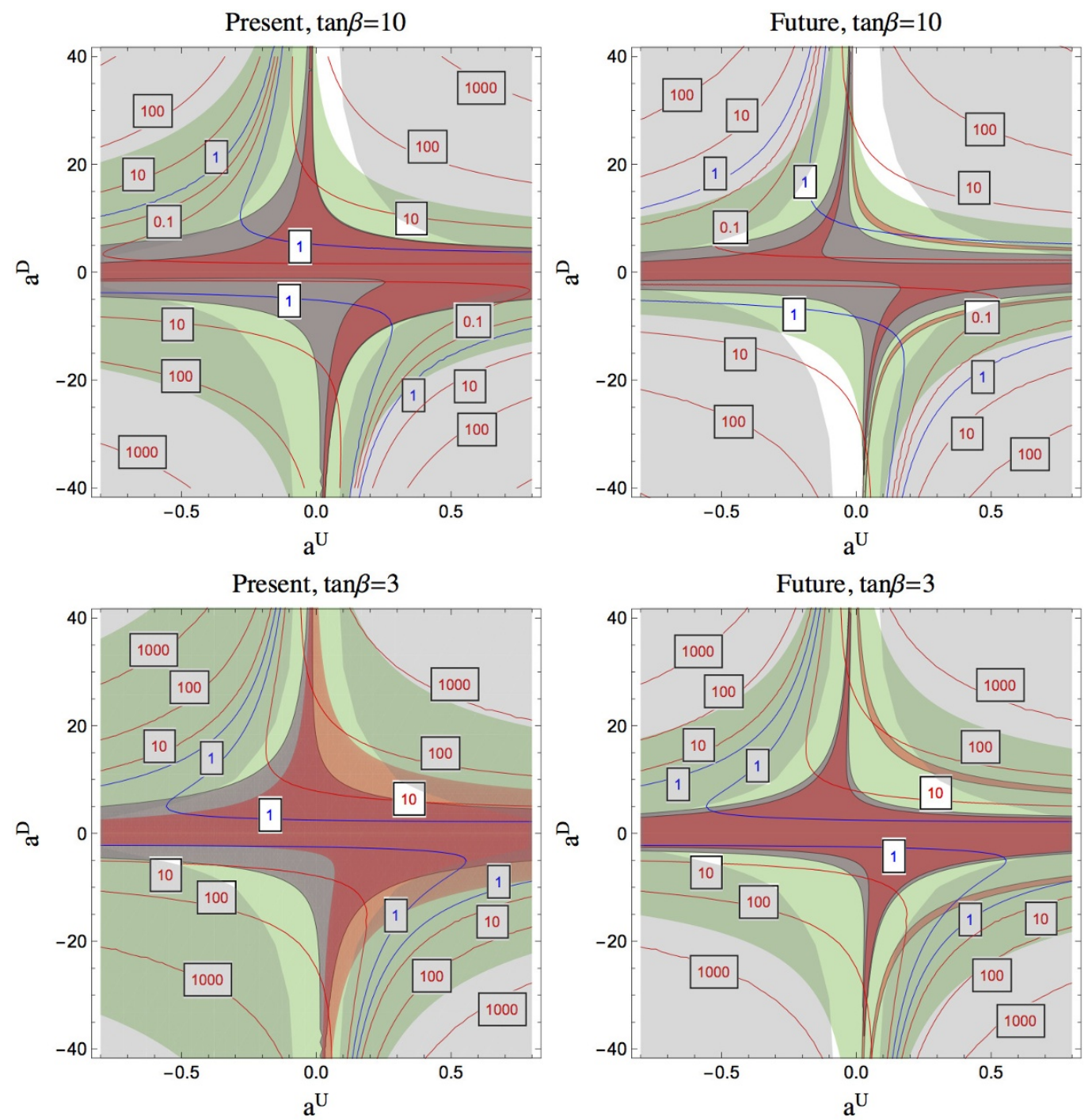

Figure 11. Summary of the constraints and predictions for the heavy Higgs phenomenology, as computed in the leading log approximation. We fix $\cos (\beta-\alpha)=0, m_{A}=m_{H}=m_{H^{ \pm}}=400 \mathrm{GeV}$, $\tan \beta=10$ (upper panels), and $\tan \beta=3$ (lower panels). The contours represent the ratio $\mathrm{BR}(H \rightarrow b \bar{b}) m_{\tau}^{2} /\left[\mathrm{BR}\left(H \rightarrow \tau^{+} \tau^{-}\right) 3 m_{b}^{2}\right]$, where $\mathbf{1}$ is the Type I and Type II $2 \mathrm{HDM}$ prediction. The reddish-brown regions are favored by all flavor constraints. The green region is favored by the measurement of $B \rightarrow \tau \nu$. Blue-gray and tan regions are favored by $B_{s}$ mixing and $B_{s} \rightarrow \mu^{+} \mu^{-}$, respectively. The gray shaded regions produce Landau poles in the Yukawa couplings below $M_{\mathrm{P}}$. The left and right panels represent the bounds as they are now and as projected for the coming years, as detailed in section 4 . 

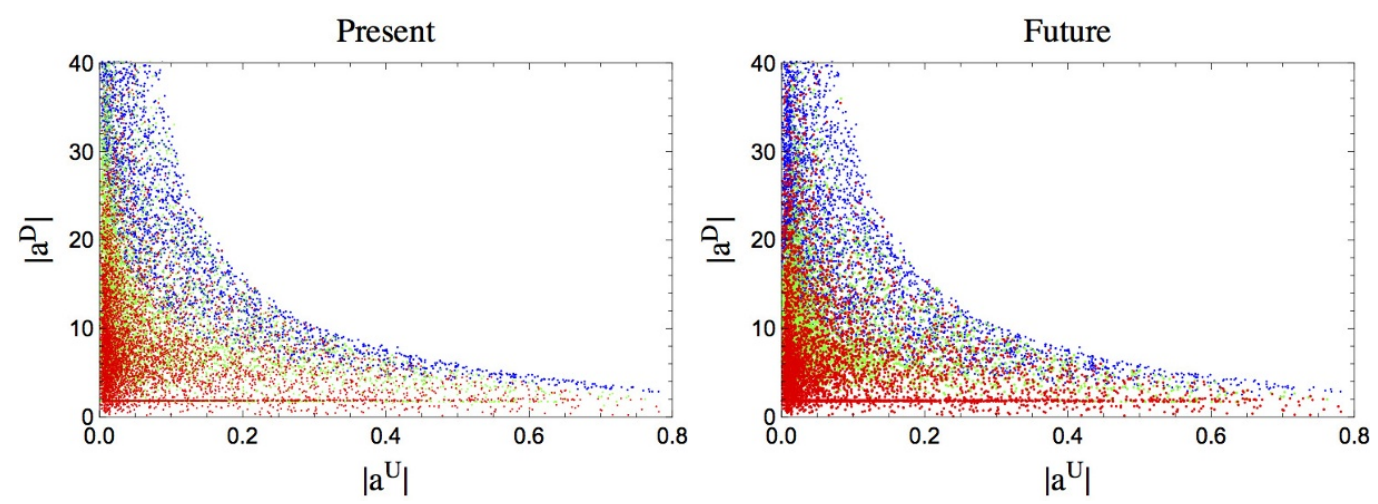

Figure 12. Result of the parameter scan using full RG running, with fixed $m_{A}=m_{H}=m_{H^{ \pm}}=$ $400 \mathrm{GeV}, \cos (\beta-\alpha)=0$, and $\tan \beta=10$. Blue points correspond to points allowed by the measurement of $B \rightarrow \tau \nu$, but not by the measurement of $B_{s}$ mixing or $B_{s} \rightarrow \mu^{+} \mu^{-}$. Green points are allowed by the measurements of $B \rightarrow \tau \nu$ and of meson mixing but not by $B_{s} \rightarrow \mu^{+} \mu^{-}$. Red points are allowed by all constraints. The left and right panels represent the bounds as they are now and as projected for the coming years, as detailed in section 4 . In the solid white region, Landau poles in the Yukawa couplings are produced below $M_{\mathrm{P}}$.

relatively weak constraint on the parameter space (green region). For values of $\tan \beta=10$ (or larger), in the region of parameter space favored by present and future flavor constraints, the ratio $m_{\tau}^{2} \mathrm{BR}(H \rightarrow \bar{b} b) / 3 m_{b}^{2} \mathrm{BR}\left(H \rightarrow \tau^{+} \tau^{-}\right)$is smaller than the ratio predicted by Type $\mathrm{I}$ and II $2 \mathrm{HDM}$ in most of the Aligned $2 \mathrm{HDM}$ parameter space. The parameter space is somewhat less constrained at lower values of $\tan \beta$, as shown in the lower panels of figure 11.

In figure 12, we present the corresponding results obtained in the numerical scan with full RG running, with fixed $\cos (\beta-\alpha)=0, m_{A}=m_{H}=m_{H^{ \pm}}=400 \mathrm{GeV}$, and $\tan \beta=10$. The qualitative features of the leading log approximation continue to hold. In particular, we again see that $B_{s} \rightarrow \mu^{+} \mu^{-}$provides the most stringent constraint on the aligned 2HDM parameter space. Note that in order to emphasize the comparison of the constraints obtained from the different $B$ physics observables in figures 11 and 12, we do not include the constraints due to the LHC searches for the heavy Higgs bosons decaying into fermion pairs in these figures. As shown in figures 4 and 5 for the heavy Higgs mass values quoted above, in the region of the Aligned 2HDM parameter space consistent with no Landau poles below $M_{\mathrm{P}}$, the current LHC limits on $H$ and $A$ production eliminate the parameter regime with $\left|a_{D}\right| \gtrsim 30-40$ and $\left|a_{U}\right| \lesssim 0.1$.

In considering the phenomenological implications of extended Higgs sectors, the most conservative approach is to impose only those constraints that are required by the current experimental data. In most $2 \mathrm{HDM}$ studies in the literature, the Yukawa couplings are assumed to be of Type I, II, X or Y. In this paper, we have argued that the current experimental data allows for a broader approach in which the Yukawa couplings are approximately aligned in flavor at the electroweak scale. The resulting phenomenology can yield some unexpected surprises. We hope that the search strategies of future Higgs studies at the LHC will be expanded to accommodate the broader phenomenological framework of the (approximately) flavor-aligned extended Higgs sector. 


\section{Acknowledgments}

H.E.H. gratefully acknowledges Paula Tuzon for numerous interactions during her two month long visit to Santa Cruz in 2010-2011. Her work on the aligned 2HDM provided inspiration for this work. S.G. thanks Wolfgang Altmannshofer for discussions. H.E.H. and E.S. are supported in part by the U.S. Department of Energy grant number DESC0010107. S.G. acknowledges support from the University of Cincinnati. S.G. and H.E.H. are grateful to the hospitality and the inspiring working atmosphere of both the Kavli Institute for Theoretical Physics in Santa Barbara, CA, supported in part by the National Science Foundation under Grant No. NSF PHY11-25915, and the Aspen Center for Physics, supported by the National Science Foundation Grant No. PHY-1066293, where some of the research reported in this work was carried out.

\section{A Review of the Higgs-fermion Yukawa couplings in the Higgs basis}

In a general $2 \mathrm{HDM}$, the Higgs fermion interactions are governed by the following interaction Lagrangian: ${ }^{25}$

$$
-\mathscr{L}_{\mathrm{Y}}=\overline{Q_{L}^{0}} \widetilde{\Phi}_{\bar{a}} \eta_{a}^{U, 0} U_{R}^{0}+\bar{Q}_{L}^{0} \Phi_{a}\left(\eta_{\bar{a}}^{D, 0}\right)^{\dagger} D_{R}^{0}+\bar{E}_{L}^{0} \Phi_{a}\left(\eta_{\bar{a}}^{E, 0}\right)^{\dagger} E_{R}^{0}+\text { h.c. },
$$

summed over $a=\bar{a}=1,2$, where $\Phi_{1,2}$ are the Higgs doublets, $\widetilde{\Phi}_{\bar{a}} \equiv i \sigma_{2} \Phi_{\bar{a}}^{*}, Q_{L}^{0}$ and $E_{L}^{0}$ are the weak isospin quark and lepton doublets, and $U_{R}^{0}, D_{R}^{0}, E_{R}^{0}$ are weak isospin quark and lepton singlets. ${ }^{26}$ Here, $Q_{L}^{0}, E_{L}^{0}, U_{R}^{0}, D_{R}^{0}, E_{R}^{0}$ denote the interaction basis states, which are vectors in the quark and lepton flavor spaces, and $\eta_{a}^{U, 0}, \eta_{a}^{D, 0}, \eta_{a}^{E, 0}$ are $3 \times 3$ matrices in quark and lepton flavor spaces.

Note that $\eta_{a}^{U, 0}$ appears undaggered in eq. (A.1), whereas the corresponding Yukawa coupling matrices for down-type fermions ( $D$ and $E$ ) appear daggered. In this convention, the transformation of the Yukawa coupling matrices under a scalar field basis change is the same for both up-type and down-type fermions. That is, under a change of basis, $\Phi_{a} \rightarrow U_{a \bar{b}} \Phi_{b}$ (which implies that $\widetilde{\Phi}_{\bar{a}} \rightarrow \widetilde{\Phi}_{\bar{b}} U_{b \bar{a}}^{\dagger}$ ), the Yukawa coupling matrices transform as $\eta_{a}^{F} \rightarrow U_{a \bar{b}} \eta_{b}^{F}$ and $\eta_{\bar{a}}^{F}{ }^{\dagger} \rightarrow \eta_{\bar{b}}^{F \dagger} U_{b \bar{a}}^{\dagger}$ (for $F=U, D$ and $E$ ), which reflects the form-invariance of $\mathscr{L}_{\mathrm{Y}}$ under the basis change.

The neutral Higgs states acquire vacuum expectation values,

$$
\left\langle\Phi_{a}^{0}\right\rangle=\frac{v \hat{v}_{a}}{\sqrt{2}},
$$

where $\hat{v}_{a} \hat{v}_{\bar{a}}^{*}=1$ and $v=246 \mathrm{GeV}$. It is also convenient to define

$$
\hat{w}_{b} \equiv \hat{v}_{\bar{a}}^{*} \epsilon_{a b},
$$

where $\epsilon_{12}=-\epsilon_{21}=1$ and $\epsilon_{11}=\epsilon_{22}=0$.

\footnotetext{
${ }^{25}$ We follow the conventions of ref. [39], in which covariance is manifest with respect to $\mathrm{U}(2)$ flavor transformations, $\Phi_{a} \rightarrow U_{a \bar{b}} \Phi_{b}$ [where $U \in \mathrm{U}(2)$ ], by implicitly summing over barred/unbarred index pairs of the same letter.

${ }^{26}$ The right and left-handed fermion fields are defined as usual: $\psi_{R, L} \equiv P_{R, L} \psi$, where $P_{R, L} \equiv \frac{1}{2}\left(1 \pm \gamma_{5}\right)$.
} 
Following refs. [37, 39], we define invariant and pseudo-invariant matrix Yukawa couplings,

$$
\kappa^{F, 0} \equiv \hat{v}_{\bar{a}}^{*} \eta_{a}^{F, 0}, \quad \quad \rho^{F, 0} \equiv \hat{w}_{\bar{a}}^{*} \eta_{a}^{F, 0},
$$

where $F=U, D$ or $E$. Inverting these equations yields

$$
\eta_{a}^{F, 0}=\kappa^{F, 0} \hat{v}_{a}+\rho^{F, 0} \hat{w}_{a}
$$

Note that under the $\mathrm{U}(2)$ transformation, $\Phi_{a} \rightarrow U_{a \bar{b}} \Phi_{b}$,

$$
\kappa^{F, 0} \text { is invariant and } \rho^{F, 0} \rightarrow(\operatorname{det} U) \rho^{F, 0} .
$$

The Higgs fields in the Higgs basis are defined by $H_{1} \equiv \hat{v}_{\bar{a}}^{*} \Phi_{a}$ and $H_{2} \equiv \hat{w}_{\bar{a}}^{*} \Phi_{a}$, which can be inverted to yield $\Phi_{a}=H_{1} \hat{v}_{a}+H_{2} w_{a}$ [39]. Rewriting eq. (A.1) in terms of the Higgs basis fields,

$$
\begin{aligned}
-\mathscr{L}_{\mathrm{Y}}= & \overline{Q_{L}^{0}}\left(\widetilde{H}_{1} \kappa^{U, 0}+\widetilde{H}_{2} \rho^{U, 0}\right) U_{R}^{0}+\bar{Q}_{L}^{0}\left(H_{1} \kappa^{D, 0 \dagger}+H_{1} \rho^{D, 0 \dagger}\right) D_{R}^{0} \\
& +\bar{E}_{L}^{0}\left(H_{1} \kappa^{E, 0 \dagger}+H_{1} \rho^{E, 0 \dagger}\right) E_{R}^{0}+\text { h.c. }
\end{aligned}
$$

The next step is to identify the quark and lepton mass-eigenstates. This is accomplished by replacing $H_{1} \rightarrow(0, v / \sqrt{2})$ and performing unitary transformations of the left and right-handed up-type and down-type fermion multiplets such that the resulting quark and charged lepton mass matrices are diagonal with non-negative entries. In more detail, we define:

$$
\begin{array}{lll}
P_{L} U=V_{L}^{U} P_{L} U^{0}, & P_{R} U=V_{R}^{U} P_{R} U^{0}, & P_{L} D=V_{L}^{D} P_{L} D^{0}, \\
P_{L} E=V_{L}^{E} P_{L} E^{0}, & P_{R} E=V_{R}^{D} P_{R} E^{0}, & P_{L} N=V_{L}^{D} P_{L} P_{R} D^{0},
\end{array}
$$

and the Cabibbo-Kobayashi-Maskawa (CKM) matrix is defined as $K \equiv V_{L}^{U} V_{L}^{D \dagger}$. Note that for the neutrino fields, we are free to choose $V_{L}^{N}=V_{L}^{E}$ since neutrinos are exactly massless in this analysis. ${ }^{27}$ In particular, the unitary matrices $V_{L}^{F}$ and $V_{R}^{F}$ (for $F=U, D$ and $E$ ) are chosen such that

$$
\begin{aligned}
& M_{U}=\frac{v}{\sqrt{2}} V_{L}^{U} \kappa^{U, 0} V_{R}^{U \dagger}=\operatorname{diag}\left(m_{u}, m_{c}, m_{t}\right), \\
& M_{D}=\frac{v}{\sqrt{2}} V_{L}^{D} \kappa^{D, 0 \dagger} V_{R}^{D \dagger}=\operatorname{diag}\left(m_{d}, m_{s}, m_{b}\right), \\
& M_{E}=\frac{v}{\sqrt{2}} V_{L}^{E} \kappa^{E, 0 \dagger} V_{R}^{E \dagger}=\operatorname{diag}\left(m_{e}, m_{\mu}, m_{\tau}\right) .
\end{aligned}
$$

It is convenient to define

$$
\begin{aligned}
\kappa^{U}=V_{L}^{U} \kappa^{U, 0} V_{R}^{U \dagger}, & \kappa^{D}=V_{R}^{D} \kappa^{D, 0} V_{L}^{D \dagger}, & \kappa^{E}=V_{R}^{D} \kappa^{E, 0} V_{L}^{E \dagger}, \\
\rho^{U}=V_{L}^{U} \rho^{U, 0} V_{R}^{U \dagger}, & \rho^{D}=V_{R}^{D} \rho^{D, 0} V_{L}^{D \dagger}, & \rho^{E}=V_{R}^{D} \rho^{E, 0} V_{L}^{E \dagger} .
\end{aligned}
$$

\footnotetext{
${ }^{27}$ Here we are ignoring the right-handed neutrino sector, which gives mass to neutrinos via the seesaw mechanism.
} 
Eq. (A.6) implies that under the $\mathrm{U}(2)$ transformation, $\Phi_{a} \rightarrow U_{a \bar{b}} \Phi_{b}$,

$$
\kappa^{F} \text { is invariant and } \rho^{F} \rightarrow(\operatorname{det} U) \rho^{F},
$$

for $F=U, D$ and $E$. Indeed, $\kappa^{F}$ is invariant since eqs. (A.9)-(A.11) imply that

$$
M_{F}=\frac{v}{\sqrt{2}} \kappa^{F},
$$

which is a physical observable. The matrices $\rho^{U}, \rho^{D}$ and $\rho^{E}$ are independent pseudoinvariant complex $3 \times 3$ matrices. The Higgs-fermion interactions given in eq. (A.7) can be rewritten in terms of the quark and lepton mass-eigenstates,

$$
\begin{aligned}
-\mathscr{L}_{\mathrm{Y}}= & \bar{U}_{L}\left(\kappa^{U} H_{1}^{0 \dagger}+\rho^{U} H_{2}^{0 \dagger}\right) U_{R}-\bar{D}_{L} K^{\dagger}\left(\kappa^{U} H_{1}^{-}+\rho^{U} H_{2}^{-}\right) U_{R} \\
& +\bar{U}_{L} K\left(\kappa^{D \dagger} H_{1}^{+}+\rho^{D \dagger} H_{2}^{+}\right) D_{R}+\bar{D}_{L}\left(\kappa^{D \dagger} H_{1}^{0}+\rho^{D \dagger} H_{2}^{0}\right) D_{R} \\
& +\bar{N}_{L}\left(\kappa^{E \dagger} H_{1}^{+}+\rho^{E \dagger} H_{2}^{+}\right) E_{R}+\bar{E}_{L}\left(\kappa^{E \dagger} H_{1}^{0}+\rho^{E \dagger} H_{2}^{0}\right) E_{R}+\text { h.c. }
\end{aligned}
$$

\section{B Renormalization group equations for the Yukawa matrices}

We first write down the renormalization group equations (RGEs) for the Yukawa matrices $\eta_{a}^{U, 0}, \eta_{a}^{D, 0}$ and $\eta_{a}^{E, 0}$. Defining $\mathcal{D} \equiv 16 \pi^{2} \mu(d / d \mu)=16 \pi^{2}(d / d t)$, the RGEs are given by [32]:

$$
\begin{aligned}
\mathcal{D} \eta_{a}^{U, 0}= & -\left(8 g_{s}^{2}+\frac{9}{4} g^{2}+\frac{17}{12} g^{\prime 2}\right) \eta_{a}^{U, 0} \\
& +\left\{3 \operatorname{Tr}\left[\eta_{a}^{U, 0}\left(\eta_{\bar{b}}^{U, 0}\right)^{\dagger}+\eta_{a}^{D, 0}\left(\eta_{\bar{b}}^{D, 0}\right)^{\dagger}\right]+\operatorname{Tr}\left[\eta_{a}^{E, 0}\left(\eta_{\bar{b}}^{E, 0}\right)^{\dagger}\right]\right\} \eta_{b}^{U, 0} \\
& -2\left(\eta_{\bar{b}}^{D, 0}\right)^{\dagger} \eta_{a}^{D, 0} \eta_{b}^{U, 0}+\eta_{a}^{U, 0}\left(\eta_{\bar{b}}^{U, 0}\right)^{\dagger} \eta_{b}^{U, 0}+\frac{1}{2}\left(\eta_{\bar{b}}^{D, 0}\right)^{\dagger} \eta_{b}^{D, 0} \eta_{a}^{U, 0}+\frac{1}{2} \eta_{b}^{U, 0}\left(\eta_{\bar{b}}^{U, 0}\right)^{\dagger} \eta_{a}^{U, 0}, \\
\mathcal{D} \eta_{a}^{D, 0}= & -\left(8 g_{s}^{2}+\frac{9}{4} g^{2}+\frac{5}{12} g^{\prime 2}\right) \eta_{a}^{D, 0} \\
& +\left\{3 \operatorname{Tr}\left[\left(\eta_{\bar{b}}^{D, 0}\right)^{\dagger} \eta_{a}^{D, 0}+\left(\eta_{\bar{b}}^{U, 0}\right)^{\dagger} \eta_{a}^{U, 0}\right]+\operatorname{Tr}\left[\left(\eta_{\bar{b}}^{E, 0}\right)^{\dagger} \eta_{a}^{E, 0}\right]\right\} \eta_{b}^{D, 0} \\
& -2 \eta_{b}^{D, 0} \eta_{a}^{U, 0}\left(\eta_{\bar{b}}^{U, 0}\right)^{\dagger}+\eta_{b}^{D, 0}\left(\eta_{\bar{b}}^{D, 0}\right)^{\dagger} \eta_{a}^{D, 0}+\frac{1}{2} \eta_{a}^{D, 0} \eta_{b}^{U, 0}\left(\eta_{\bar{b}}^{U, 0}\right)^{\dagger}+\frac{1}{2} \eta_{a}^{D, 0}\left(\eta_{\bar{b}}^{D, 0}\right)^{\dagger} \eta_{b}^{D, 0}, \\
\mathcal{D} \eta_{a}^{E, 0}= & -\left(\frac{9}{4} g^{2}+\frac{15}{4} g^{\prime 2}\right) \eta_{a}^{E, 0}+\left\{3 \operatorname{Tr}\left[\left(\eta_{\bar{b}}^{D, 0}\right)^{\dagger} \eta_{a}^{D, 0}+\left(\eta_{\bar{b}}^{U, 0}\right)^{\dagger} \eta_{a}^{U, 0}\right]+\operatorname{Tr}\left[\left(\eta_{\bar{b}}^{E, 0}\right)^{\dagger} \eta_{a}^{E, 0}\right]\right\} \eta_{b}^{E, 0} \\
& +\eta_{b}^{E, 0}\left(\eta_{\bar{b}}^{E, 0}\right)^{\dagger} \eta_{a}^{E, 0}+\frac{1}{2} \eta_{a}^{E, 0}\left(\eta_{\bar{b}}^{E, 0}\right)^{\dagger} \eta_{b}^{E, 0} .
\end{aligned}
$$

The RGEs above are true for any basis choice. Thus, they must also be true in the Higgs basis in which $\hat{v}=(1,0)$ and $\hat{w}=(0,1)$. In this case, we can simply choose $\eta_{1}^{F, 0}=\kappa^{F, 0}$ and $\eta_{2}^{F, 0}=\rho^{F, 0}$ to obtain the RGEs for the $\kappa^{F, 0}$ and $\rho^{F, 0}$. Alternatively, we can multiply eqs. (B.1)-(B.3) first by $\hat{v}_{a}^{*}$ and then by $\hat{w}_{a}^{*}$. Expanding $\eta_{\bar{a}}^{\dagger}$, which appears on the right-hand sides of eqs. (B.1)-(B.3), in terms of $\kappa^{\dagger}$ and $\rho^{\dagger}$ using eq. (A.5), we again obtain the RGEs for the $\kappa^{F, 0}$ and $\rho^{F, 0}$. Of course, both methods yield the same result, since the diagonalization matrices employed in eqs. (A.9)-(A.11) are defined as those that bring the mass matrices to their diagonal form at the electroweak scale. No scale dependence is assumed in the diagonalization matrices, and as such they are not affected by the operators $\mathcal{D}$. 


$$
\begin{aligned}
& \mathcal{D} \kappa^{U, 0}=-\left(8 g_{s}^{2}+\frac{9}{4} g^{2}+\frac{17}{12} g^{\prime 2}\right) \kappa^{U, 0}+\left\{3 \operatorname{Tr}\left[\kappa^{U, 0} \kappa^{U, 0 \dagger}+\kappa^{D, 0} \kappa^{D, 0 \dagger}\right]+\operatorname{Tr}\left[\kappa^{E, 0} \kappa^{E, 0 \dagger}\right]\right\} \kappa^{U, 0} \\
& +\left\{3 \operatorname{Tr}\left[\kappa^{U, 0} \rho^{U, 0 \dagger}+\kappa^{D, 0} \rho^{D, 0 \dagger}\right]+\operatorname{Tr}\left[\kappa^{E, 0} \rho^{E, 0 \dagger}\right]\right\} \rho^{U, 0}-2\left(\kappa^{D, 0 \dagger} \kappa^{D, 0} \kappa^{U, 0}+\rho^{D, 0 \dagger} \kappa^{D, 0} \rho^{U, 0}\right) \\
& +\kappa^{U, 0}\left(\kappa^{U, 0 \dagger} \kappa^{U, 0}+\rho^{U, 0 \dagger} \rho^{U, 0}\right)+\frac{1}{2}\left(\kappa^{D, 0 \dagger} \kappa^{D, 0}+\rho^{D, 0 \dagger} \rho^{D, 0}\right) \kappa^{U, 0} \\
& +\frac{1}{2}\left(\kappa^{U, 0} \kappa^{U, 0 \dagger}+\rho^{U, 0} \rho^{U, 0 \dagger}\right) \kappa^{U, 0}, \\
& \mathcal{D} \rho^{U, 0}=-\left(8 g_{s}^{2}+\frac{9}{4} g^{2}+\frac{17}{12} g^{\prime 2}\right) \rho^{U, 0}+\left\{3 \operatorname{Tr}\left[\rho^{U, 0} \kappa^{U, 0 \dagger}+\rho^{D, 0} \kappa^{D, 0 \dagger}\right]+\operatorname{Tr}\left[\rho^{E, 0} \kappa^{E, 0 \dagger}\right]\right\} \kappa^{U, 0} \\
& +\left\{3 \operatorname{Tr}\left[\rho^{U, 0} \rho^{U, 0 \dagger}+\rho^{D, 0} \rho^{D, 0 \dagger}\right]+\operatorname{Tr}\left[\rho^{E, 0} \rho^{E, 0 \dagger}\right]\right\} \rho^{U, 0}-2\left(\kappa^{D, 0 \dagger} \rho^{D, 0} \kappa^{U, 0}+\rho^{D, 0 \dagger} \rho^{D, 0} \rho^{U, 0}\right) \\
& +\rho^{U, 0}\left(\kappa^{U, 0 \dagger} \kappa^{U, 0}+\rho^{U, 0 \dagger} \rho^{U, 0}\right)+\frac{1}{2}\left(\kappa^{D, 0 \dagger} \kappa^{D, 0}+\rho^{D, 0 \dagger} \rho^{D, 0}\right) \rho^{U, 0} \\
& +\frac{1}{2}\left(\kappa^{U, 0} \kappa^{U, 0 \dagger}+\rho^{U, 0} \rho^{U, 0 \dagger}\right) \rho^{U, 0}, \\
& \mathcal{D} \kappa^{D, 0}=-\left(8 g_{s}^{2}+\frac{9}{4} g^{2}+\frac{5}{12} g^{\prime 2}\right) \kappa^{D, 0}+\left\{3 \operatorname{Tr}\left[\kappa^{D, 0 \dagger} \kappa^{D, 0}+\kappa^{U, 0 \dagger} \kappa^{U, 0}\right]+\operatorname{Tr}\left[\kappa^{E, 0 \dagger} \kappa^{E, 0}\right]\right\} \kappa^{D, 0} \\
& +\left\{3 \operatorname{Tr}\left[\rho^{D, 0 \dagger} \kappa^{D, 0}+\rho^{U, 0 \dagger} \kappa^{U, 0}\right]+\operatorname{Tr}\left[\rho^{E, 0 \dagger} \kappa^{E, 0}\right]\right\} \rho^{D, 0}-2\left(\kappa^{D, 0} \kappa^{U, 0} \kappa^{U, 0 \dagger}\right. \\
& \left.+\rho^{D, 0} \kappa^{U, 0} \rho^{U, 0 \dagger}\right)+\left(\kappa^{D, 0} \kappa^{D, 0 \dagger}+\rho^{D, 0} \rho^{D, 0 \dagger}\right) \kappa^{D, 0}+\frac{1}{2} \kappa^{D, 0}\left(\kappa^{U, 0} \kappa^{U, 0 \dagger}+\rho^{U, 0} \rho^{U, 0 \dagger}\right) \\
& +\frac{1}{2} \kappa^{D, 0}\left(\kappa^{D, 0 \dagger} \kappa^{D, 0}+\rho^{D, 0 \dagger} \rho^{D, 0}\right), \\
& \mathcal{D} \rho^{D, 0}=-\left(8 g_{s}^{2}+\frac{9}{4} g^{2}+\frac{5}{12} g^{\prime 2}\right) \rho^{D, 0}+\left\{3 \operatorname{Tr}\left[\kappa^{D, 0 \dagger} \rho^{D, 0}+\kappa^{U, 0 \dagger} \rho^{U, 0}\right]+\operatorname{Tr}\left[\kappa^{E, 0 \dagger} \rho^{E, 0}\right]\right\} \kappa^{D, 0} \\
& +\left\{3 \operatorname{Tr}\left[\rho^{D, 0 \dagger} \rho^{D, 0}+\rho^{U, 0 \dagger} \rho^{U, 0}\right]+\operatorname{Tr}\left[\rho^{E, 0 \dagger} \rho^{E, 0}\right]\right\} \rho^{D, 0}-2\left(\kappa^{D, 0} \rho^{U, 0} \kappa^{U, 0 \dagger}+\rho^{D, 0} \rho^{U, 0} \rho^{U, 0 \dagger}\right) \\
& +\left(\kappa^{D, 0} \kappa^{D, 0 \dagger}+\rho^{D, 0} \rho^{D, 0 \dagger}\right) \rho^{D, 0}+\frac{1}{2} \rho^{D, 0}\left(\kappa^{U, 0} \kappa^{U, 0 \dagger}+\rho^{U, 0} \rho^{U, 0 \dagger}\right) \\
& +\frac{1}{2} \rho^{D, 0}\left(\kappa^{D, 0 \dagger} \kappa^{D, 0}+\rho^{D, 0 \dagger} \rho^{D, 0}\right), \\
& \mathcal{D} \kappa^{E, 0}=-\left(\frac{9}{4} g^{2}+\frac{15}{4} g^{\prime 2}\right) \kappa^{E, 0}+\left\{3 \operatorname{Tr}\left[\kappa^{D, 0 \dagger} \kappa^{D, 0}+\kappa^{U, 0 \dagger} \kappa^{U, 0}\right]+\operatorname{Tr}\left[\kappa^{E, 0^{\dagger}} \kappa^{E, 0}\right]\right\} \kappa^{E, 0} \\
& +\left\{3 \operatorname{Tr}\left[\rho^{D, 0 \dagger} \kappa^{D, 0}+\rho^{U, 0 \dagger} \kappa^{U, 0}\right]+\operatorname{Tr}\left[\rho^{E, 0^{\dagger}} \kappa^{E, 0}\right]\right\} \rho^{E, 0} \\
& +\left(\kappa^{E, 0} \kappa^{E, 0 \dagger}+\rho^{E, 0} \rho^{E, 0 \dagger}\right) \kappa^{E, 0}+\frac{1}{2} \kappa^{E, 0}\left(\kappa^{E, 0 \dagger} \kappa^{E, 0}+\rho^{E, 0 \dagger} \rho^{E, 0}\right), \\
& \mathcal{D} \rho^{E, 0}=-\left(\frac{9}{4} g^{2}+\frac{15}{4} g^{\prime 2}\right) \rho^{E, 0}+\left\{3 \operatorname{Tr}\left[\kappa^{D, 0 \dagger} \rho^{D, 0}+\kappa^{U, 0 \dagger} \rho^{U, 0}\right]+\operatorname{Tr}\left[\kappa^{E, 0^{\dagger}} \rho^{E, 0}\right]\right\} \kappa^{E, 0} \\
& +\left\{3 \operatorname{Tr}\left[\rho^{D, 0 \dagger} \rho^{D, 0}+\rho^{U, 0 \dagger} \rho^{U, 0}\right]+\operatorname{Tr}\left[\rho^{E, 0^{\dagger}} \rho^{E, 0}\right]\right\} \rho^{E, 0} \\
& +\left(\kappa^{E} \kappa^{E, 0 \dagger}+\rho^{E, 0} \rho^{E, 0 \dagger}\right) \rho^{E, 0}+\frac{1}{2} \rho^{E, 0}\left(\kappa^{E, 0 \dagger} \kappa^{E, 0}+\rho^{E, 0 \dagger} \rho^{E, 0}\right) .
\end{aligned}
$$


Using eqs. (A.12) and (A.13), we immediately obtain the RGEs for the $\kappa^{F}$ and $\rho^{F}$. Schematically, we shall write,

$$
\mathcal{D} \kappa^{F}=\beta_{\kappa^{F}}, \quad \mathcal{D} \rho^{F}=\beta_{\rho^{F}},
$$

for $F=U, D$ and $E$. Explicitly, the corresponding $\beta$-functions at one-loop order are given by,

$$
\begin{aligned}
& \mathcal{D} \kappa^{U}=-\left(8 g_{s}^{2}+\frac{9}{4} g^{2}+\frac{17}{12} g^{\prime 2}\right) \kappa^{U}+\left\{3 \operatorname{Tr}\left[\kappa^{U} \kappa^{U \dagger}+\kappa^{D} \kappa^{D \dagger}\right]+\operatorname{Tr}\left[\kappa^{E} \kappa^{E \dagger}\right]\right\} \kappa^{U} \\
& +\left\{3 \operatorname{Tr}\left[\kappa^{U} \rho^{U \dagger}+\kappa^{D} \rho^{D \dagger}\right]+\operatorname{Tr}\left[\kappa^{E} \rho^{E \dagger}\right]\right\} \rho^{U}-2 K\left(\kappa^{D \dagger} \kappa^{D} K^{\dagger} \kappa^{U}+\rho^{D \dagger} \kappa^{D} K^{\dagger} \rho^{U}\right) \\
& +\kappa^{U}\left(\kappa^{U \dagger} \kappa^{U}+\rho^{U \dagger} \rho^{U}\right)+\frac{1}{2} K\left(\kappa^{D \dagger} \kappa^{D}+\rho^{D \dagger} \rho^{D}\right) K^{\dagger} \kappa^{U}+\frac{1}{2}\left(\kappa^{U} \kappa^{U \dagger}+\rho^{U} \rho^{U \dagger}\right) \kappa^{U}, \\
& \mathcal{D} \rho^{U}=-\left(8 g_{s}^{2}+\frac{9}{4} g^{2}+\frac{17}{12} g^{\prime 2}\right) \rho^{U}+\left\{3 \operatorname{Tr}\left[\rho^{U} \kappa^{U \dagger}+\rho^{D} \kappa^{D \dagger}\right]+\operatorname{Tr}\left[\rho^{E} \kappa^{E \dagger}\right]\right\} \kappa^{U} \\
& +\left\{3 \operatorname{Tr}\left[\rho^{U} \rho^{U \dagger}+\rho^{D} \rho^{D \dagger}\right]+\operatorname{Tr}\left[\rho^{E} \rho^{E \dagger}\right]\right\} \rho^{U}-2 K\left(\kappa^{D \dagger} \rho^{D} K^{\dagger} \kappa^{U}+\rho^{D \dagger} \rho^{D} K^{\dagger} \rho^{U}\right) \\
& +\rho^{U}\left(\kappa^{U \dagger} \kappa^{U}+\rho^{U \dagger} \rho^{U}\right)+\frac{1}{2} K\left(\kappa^{D \dagger} \kappa^{D}+\rho^{D \dagger} \rho^{D}\right) K^{\dagger} \rho^{U}+\frac{1}{2}\left(\kappa^{U} \kappa^{U \dagger}+\rho^{U} \rho^{U \dagger}\right) \rho^{U}, \\
& \mathcal{D} \kappa^{D}=-\left(8 g_{s}^{2}+\frac{9}{4} g^{2}+\frac{5}{12} g^{\prime 2}\right) \kappa^{D}+\left\{3 \operatorname{Tr}\left[\kappa^{D \dagger} \kappa^{D}+\kappa^{U \dagger} \kappa^{U}\right]+\operatorname{Tr}\left[\kappa^{E \dagger} \kappa^{E}\right]\right\} \kappa^{D} \\
& +\left\{3 \operatorname{Tr}\left[\rho^{D \dagger} \kappa^{D}+\rho^{U \dagger} \kappa^{U}\right]+\operatorname{Tr}\left[\rho^{E \dagger} \kappa^{E}\right]\right\} \rho^{D}-2\left(\kappa^{D} K^{\dagger} \kappa^{U} \kappa^{U \dagger}+\rho^{D} K^{\dagger} \kappa^{U} \rho^{U \dagger}\right) K \\
& +\left(\kappa^{D} \kappa^{D \dagger}+\rho^{D} \rho^{D \dagger}\right) \kappa^{D}+\frac{1}{2} \kappa^{D} K^{\dagger}\left(\kappa^{U} \kappa^{U \dagger}+\rho^{U} \rho^{U \dagger}\right) K+\frac{1}{2} \kappa^{D}\left(\kappa^{D \dagger} \kappa^{D}+\rho^{D \dagger} \rho^{D}\right), \\
& \mathcal{D} \rho^{D}=-\left(8 g_{s}^{2}+\frac{9}{4} g^{2}+\frac{5}{12} g^{\prime 2}\right) \rho^{D}+\left\{3 \operatorname{Tr}\left[\kappa^{D \dagger} \rho^{D}+\kappa^{U \dagger} \rho^{U}\right]+\operatorname{Tr}\left[\kappa^{E \dagger} \rho^{E}\right]\right\} \kappa^{D} \\
& +\left\{3 \operatorname{Tr}\left[\rho^{D \dagger} \rho^{D}+\rho^{U \dagger} \rho^{U}\right]+\operatorname{Tr}\left[\rho^{E \dagger} \rho^{E}\right]\right\} \rho^{D}-2\left(\kappa^{D} K^{\dagger} \rho^{U} \kappa^{U \dagger}+\rho^{D} K^{\dagger} \rho^{U} \rho^{U \dagger}\right) K \\
& +\left(\kappa^{D} \kappa^{D \dagger}+\rho^{D} \rho^{D \dagger}\right) \rho^{D}+\frac{1}{2} \rho^{D} K^{\dagger}\left(\kappa^{U} \kappa^{U \dagger}+\rho^{U} \rho^{U \dagger}\right) K+\frac{1}{2} \rho^{D}\left(\kappa^{D \dagger} \kappa^{D}+\rho^{D \dagger} \rho^{D}\right), \\
& \mathcal{D} \kappa^{E}=-\left(\frac{9}{4} g^{2}+\frac{15}{4} g^{\prime 2}\right) \kappa^{E}+\left\{3 \operatorname{Tr}\left[\kappa^{D \dagger} \kappa^{D}+\kappa^{U \dagger} \kappa^{U}\right]+\operatorname{Tr}\left[\kappa^{E^{\dagger}} \kappa^{E}\right]\right\} \kappa^{E} \\
& +\left\{3 \operatorname{Tr}\left[\rho^{D \dagger} \kappa^{D}+\rho^{U \dagger} \kappa^{U}\right]+\operatorname{Tr}\left[\rho^{E^{\dagger}} \kappa^{E}\right]\right\} \rho^{E} \\
& +\left(\kappa^{E} \kappa^{E \dagger}+\rho^{E} \rho^{E \dagger}\right) \kappa^{E}+\frac{1}{2} \kappa^{E}\left(\kappa^{E \dagger} \kappa^{E}+\rho^{E \dagger} \rho^{E}\right), \\
& \mathcal{D} \rho^{E}=-\left(\frac{9}{4} g^{2}+\frac{15}{4} g^{\prime 2}\right) \rho^{E}+\left\{3 \operatorname{Tr}\left[\kappa^{D \dagger} \rho^{D}+\kappa^{U \dagger} \rho^{U}\right]+\operatorname{Tr}\left[\kappa^{E^{\dagger}} \rho^{E}\right]\right\} \kappa^{E} \\
& +\left\{3 \operatorname{Tr}\left[\rho^{D \dagger} \rho^{D}+\rho^{U \dagger} \rho^{U}\right]+\operatorname{Tr}\left[\rho^{E^{\dagger}} \rho^{E}\right]\right\} \rho^{E} \\
& +\left(\kappa^{E} \kappa^{E \dagger}+\rho^{E} \rho^{E \dagger}\right) \rho^{E}+\frac{1}{2} \rho^{E}\left(\kappa^{E \dagger} \kappa^{E}+\rho^{E \dagger} \rho^{E}\right) .
\end{aligned}
$$

For the numerical analysis of the RGEs, it is convenient to define

$$
\widetilde{\kappa}^{D} \equiv \kappa^{D} K^{\dagger}, \quad \widetilde{\rho}^{D} \equiv \rho^{D} K^{\dagger},
$$


keeping in mind that the (unitary) CKM matrix $K$ is defined at the electroweak scale and thus is not taken to be a running quantity. The RGEs given in eqs. (B.11)-(B.16) can now be rewritten by taking $\kappa^{D} \rightarrow \widetilde{\kappa}^{D}, \rho^{D} \rightarrow \widetilde{\rho}^{D}$ and $K \rightarrow \mathbb{1}$. The advantage of the RGEs written in this latter form is that the CKM matrix $K$ no longer appears explicitly in the differential equations, and enters only in the initial condition of $\widetilde{\kappa}^{D}$ at the low scale [cf. eq. (2.62)],

$$
\widetilde{\kappa}^{D}\left(\Lambda_{H}\right)=\sqrt{2} M_{D}\left(\Lambda_{H}\right) K^{\dagger} / v .
$$

In particular, the high scale boundary condition given by eq. (2.63) also applies to $\widetilde{\kappa}^{D}$ and $\widetilde{\rho}^{D}$, i.e.,

$$
\widetilde{\rho}^{D}(\Lambda)=a^{D} \widetilde{\kappa}^{D}(\Lambda) .
$$

Open Access. This article is distributed under the terms of the Creative Commons Attribution License (CC-BY 4.0), which permits any use, distribution and reproduction in any medium, provided the original author(s) and source are credited.

\section{References}

[1] ATLAS collaboration, Observation of a new particle in the search for the Standard Model Higgs boson with the ATLAS detector at the LHC, Phys. Lett. B 716 (2012) 1 [arXiv: 1207.7214$]$ [INSPIRE].

[2] CMS collaboration, Observation of a new boson at a mass of $125 \mathrm{GeV}$ with the CMS experiment at the LHC, Phys. Lett. B 716 (2012) 30 [arXiv:1207.7235] [INSPIRE].

[3] ATLAS and CMS collaborations, Measurements of the Higgs boson production and decay rates and constraints on its couplings from a combined ATLAS and CMS analysis of the LHC pp collision data at $\sqrt{s}=7$ and 8 TeV, JHEP 08 (2016) 045 [arXiv:1606.02266] [INSPIRE].

[4] P. Fayet, Supergauge invariant extension of the Higgs mechanism and a model for the electron and its neutrino, Nucl. Phys. B 90 (1975) 104 [INSPIRE].

[5] K. Inoue, A. Kakuto, H. Komatsu and S. Takeshita, Low-energy parameters and particle masses in a supersymmetric grand unified model, Prog. Theor. Phys. 67 (1982) 1889 [INSPIRE].

[6] R.A. Flores and M. Sher, Higgs masses in the standard, multi-Higgs and supersymmetric models, Annals Phys. 148 (1983) 95 [INSPIRE].

[7] J.F. Gunion and H.E. Haber, Higgs bosons in supersymmetric models. 1, Nucl. Phys. B 272 (1986) 1 [Erratum ibid. B 402 (1993) 567] [InSPIRE].

[8] L. Fromme, S.J. Huber and M. Seniuch, Baryogenesis in the two-Higgs doublet model, JHEP 11 (2006) 038 [hep-ph/0605242] [INSPIRE].

[9] F. Bezrukov, M. Yu. Kalmykov, B.A. Kniehl and M. Shaposhnikov, Higgs boson mass and new physics, JHEP 10 (2012) 140 [arXiv:1205.2893] [INSPIRE].

[10] G. Degrassi et al., Higgs mass and vacuum stability in the Standard Model at NNLO, JHEP 08 (2012) 098 [arXiv: 1205.6497] [INSPIRE].

[11] D. Buttazzo et al., Investigating the near-criticality of the Higgs boson, JHEP 12 (2013) 089 [arXiv: 1307.3536] [INSPIRE]. 
[12] J. Elias-Miro, J.R. Espinosa, G.F. Giudice, H.M. Lee and A. Strumia, Stabilization of the electroweak vacuum by a scalar threshold effect, JHEP 06 (2012) 031 [arXiv:1203.0237] [INSPIRE].

[13] O. Lebedev, On stability of the electroweak vacuum and the Higgs portal, Eur. Phys. J. C 72 (2012) 2058 [arXiv: 1203.0156] [INSPIRE].

[14] G.M. Pruna and T. Robens, Higgs singlet extension parameter space in the light of the LHC discovery, Phys. Rev. D 88 (2013) 115012 [arXiv:1303.1150] [INSPIRE].

[15] R. Costa, A.P. Morais, M.O.P. Sampaio and R. Santos, Two-loop stability of a complex singlet extended Standard Model, Phys. Rev. D 92 (2015) 025024 [arXiv:1411.4048] [INSPIRE].

[16] N. Chakrabarty, U.K. Dey and B. Mukhopadhyaya, High-scale validity of a two-Higgs doublet scenario: a study including LHC data, JHEP 12 (2014) 166 [arXiv:1407.2145] [INSPIRE].

[17] D. Das and I. Saha, Search for a stable alignment limit in two-Higgs-doublet models, Phys. Rev. D 91 (2015) 095024 [arXiv: 1503.02135] [INSPIRE].

[18] P. Ferreira, H.E. Haber and E. Santos, Preserving the validity of the two-Higgs doublet model up to the Planck scale, Phys. Rev. D 92 (2015) 033003 [Erratum ibid. D 94 (2016) 059903] [arXiv: 1505.04001] [INSPIRE].

[19] D. Chowdhury and O. Eberhardt, Global fits of the two-loop renormalized two-Higgs-doublet model with soft $Z_{2}$ breaking, JHEP 11 (2015) 052 [arXiv:1503.08216] [INSPIRE].

[20] G.C. Branco, P.M. Ferreira, L. Lavoura, M.N. Rebelo, M. Sher and J.P. Silva, Theory and phenomenology of two-Higgs-doublet models, Phys. Rept. 516 (2012) 1 [arXiv:1106.0034] [INSPIRE].

[21] M. Misiak and M. Steinhauser, Weak radiative decays of the $B$ meson and bounds on $M_{H^{ \pm}}$ in the two-Higgs-doublet model, Eur. Phys. J. C 77 (2017) 201 [arXiv:1702.04571] [INSPIRE].

[22] S.L. Glashow and S. Weinberg, Natural conservation laws for neutral currents, Phys. Rev. D 15 (1977) 1958 [INSPIRE].

[23] E.A. Paschos, Diagonal neutral currents, Phys. Rev. D 15 (1977) 1966 [InSPIRE].

[24] H.E. Haber, G.L. Kane and T. Sterling, The fermion mass scale and possible effects of Higgs bosons on experimental observables, Nucl. Phys. B 161 (1979) 493 [INSPIRE].

[25] L.J. Hall and M.B. Wise, Flavor changing Higgs-boson couplings, Nucl. Phys. B 187 (1981) 397 [INSPIRE].

[26] J.F. Donoghue and L.F. Li, Properties of charged Higgs bosons, Phys. Rev. D 19 (1979) 945 [INSPIRE].

[27] V.D. Barger, J.L. Hewett and R.J.N. Phillips, New constraints on the charged Higgs sector in two Higgs doublet models, Phys. Rev. D 41 (1990) 3421 [INSPIRE].

[28] M. Aoki, S. Kanemura, K. Tsumura and K. Yagyu, Models of Yukawa interaction in the two Higgs doublet model and their collider phenomenology, Phys. Rev. D 80 (2009) 015017 [arXiv:0902 .4665] [INSPIRE]. 
[29] A.J. Buras, M.V. Carlucci, S. Gori and G. Isidori, Higgs-mediated FCNCs: natural flavour conservation vs. minimal flavour violation, JHEP 10 (2010) 009 [arXiv:1005.5310] [INSPIRE].

[30] A. Pich and P. Tuzon, Yukawa alignment in the two-Higgs-doublet model, Phys. Rev. D 80 (2009) 091702 [arXiv:0908.1554] [INSPIRE].

[31] F.J. Botella, G.C. Branco, A.M. Coutinho, M.N. Rebelo and J.I. Silva-Marcos, Natural quasi-alignment with two Higgs doublets and RGE stability, Eur. Phys. J. C 75 (2015) 286 [arXiv: 1501.07435] [INSPIRE].

[32] P.M. Ferreira, L. Lavoura and J.P. Silva, Renormalization-group constraints on Yukawa alignment in multi-Higgs-doublet models, Phys. Lett. B 688 (2010) 341 [arXiv:1001.2561] [INSPIRE].

[33] S. Knapen and D.J. Robinson, Disentangling mass and mixing hierarchies, Phys. Rev. Lett. 115 (2015) 161803 [arXiv: 1507.00009] [INSPIRE].

[34] F.J. Botella, G.C. Branco, M. Nebot and M.N. Rebelo, Flavour changing Higgs couplings in a class of two Higgs doublet models, Eur. Phys. J. C 76 (2016) 161 [arXiv:1508.05101] [INSPIRE].

[35] G. D'Ambrosio, G.F. Giudice, G. Isidori and A. Strumia, Minimal flavor violation: an effective field theory approach, Nucl. Phys. B 645 (2002) 155 [hep-ph/0207036] [InSPIRE].

[36] C.B. Braeuninger, A. Ibarra and C. Simonetto, Radiatively induced flavour violation in the general two-Higgs doublet model with Yukawa alignment, Phys. Lett. B 692 (2010) 189 [arXiv: 1005.5706] [INSPIRE].

[37] S. Davidson and H.E. Haber, Basis-independent methods for the two-Higgs-doublet model, Phys. Rev. D 72 (2005) 035004 [Erratum ibid. D 72 (2005) 099902] [hep-ph/0504050] [INSPIRE].

[38] G.C. Branco, L. Lavoura and J.P. Silva, CP violation, Oxford University Press, Oxford U.K., (1999) [INSPIRE].

[39] H.E. Haber and D. O'Neil, Basis-independent methods for the two-Higgs-doublet model II. The significance of $\tan \beta$, Phys. Rev. D 74 (2006) 015018 [Erratum ibid. D 74 (2006) 059905] [hep-ph/0602242] [INSPIRE].

[40] H.E. Haber and D. O'Neil, Basis-independent methods for the two-Higgs-doublet model III. The CP-conserving limit, custodial symmetry and the oblique parameters $S, T, U$, Phys. Rev. D 83 (2011) 055017 [arXiv: 1011.6188] [INSPIRE].

[41] P. Minkowski, $\mu \rightarrow$ er at a rate of one out of $10^{9}$ muon decays?, Phys. Lett. B 67 (1977) 421 [INSPIRE].

[42] M. Gell-Mann, P. Ramond and R. Slansky, Complex spinors and unified theories, Conf. Proc. C 790927 (1979) 315 [arXiv:1306.4669] [inSPIRE].

[43] T. Yanagida, Horizontal symmetry and masses of neutrinos, Prog. Theor. Phys. 64 (1980) 1103 [INSPIRE].

[44] R.N. Mohapatra and G. Senjanović, Neutrino mass and spontaneous parity violation, Phys. Rev. Lett. 44 (1980) 912 [INSPIRE].

[45] R.N. Mohapatra and G. Senjanović, Neutrino masses and mixings in gauge models with spontaneous parity violation, Phys. Rev. D 23 (1981) 165 [INSPIRE]. 
[46] J.F. Gunion and H.E. Haber, The CP conserving two Higgs doublet model: the approach to the decoupling limit, Phys. Rev. D 67 (2003) 075019 [hep-ph/0207010] [INSPIRE].

[47] N. Craig, J. Galloway and S. Thomas, Searching for signs of the second Higgs doublet, arXiv: 1305.2424 [INSPIRE].

[48] D.M. Asner et al., ILC Higgs white paper, in Proceedings, Community Summer Study 2013: Snowmass on the Mississippi (CSS2013), Minneapolis MN U.S.A., 29 July-6 August 2013 [arXiv: 1310.0763] [INSPIRE].

[49] M. Carena, I. Low, N.R. Shah and C.E.M. Wagner, Impersonating the Standard Model Higgs boson: alignment without decoupling, JHEP 04 (2014) 015 [arXiv:1310.2248] [INSPIRE].

[50] H.E. Haber, The Higgs data and the decoupling limit, in Proceedings, $1^{\text {st }}$ Toyama International Workshop on Higgs as a Probe of New Physics 2013 (HPNP2013), Toyama Japan, 13-16 February 2013 [arXiv: 1401.0152] [INSPIRE].

[51] P.S. Bhupal Dev and A. Pilaftsis, Maximally symmetric two Higgs doublet model with natural Standard Model alignment, JHEP 12 (2014) 024 [Erratum ibid. 11 (2015) 147] [arXiv: 1408.3405] [INSPIRE].

[52] A. Pilaftsis, Symmetries for Standard Model alignment in multi-Higgs doublet models, Phys. Rev. D 93 (2016) 075012 [arXiv: 1602.02017] [INSPIRE].

[53] H.E. Haber and Y. Nir, Multiscalar models with a high-energy scale, Nucl. Phys. B 335 (1990) 363 [INSPIRE].

[54] J.F. Gunion and H.E. Haber, Conditions for CP-violation in the general two-Higgs-doublet model, Phys. Rev. D 72 (2005) 095002 [hep-ph/0506227] [INSPIRE].

[55] K.G. Chetyrkin, J.H. Kuhn and M. Steinhauser, RunDec: a Mathematica package for running and decoupling of the strong coupling and quark masses, Comput. Phys. Commun. 133 (2000) 43 [hep-ph/0004189] [INSPIRE].

[56] F. Herren and M. Steinhauser, Version 3 of RunDec and CRunDec, arXiv:1703.03751 [INSPIRE].

[57] Particle Data Group collaboration, C. Patrignani et al., Review of particle physics, Chin. Phys. C 40 (2016) 100001 [inSPIRE].

[58] P. Marquard, A.V. Smirnov, V.A. Smirnov and M. Steinhauser, Quark mass relations to four-loop order in perturbative QCD, Phys. Rev. Lett. 114 (2015) 142002 [arXiv: 1502 .01030] [INSPIRE].

[59] J. Bijnens, J. Lu and J. Rathsman, Constraining general two Higgs doublet models by the evolution of Yukawa couplings, JHEP 05 (2012) 118 [arXiv:1111.5760] [INSPIRE].

[60] M. Jung, A. Pich and P. Tuzon, Charged-Higgs phenomenology in the aligned two-Higgs-doublet model, JHEP 11 (2010) 003 [arXiv:1006.0470] [INSPIRE].

[61] ATLAS collaboration, Constraints on new phenomena via Higgs boson couplings and invisible decays with the ATLAS detector, JHEP 11 (2015) 206 [arXiv:1509.00672] [INSPIRE].

[62] CMS collaboration, Summary results of high mass BSM Higgs searches using CMS run-I data, CMS-PAS-HIG-16-007, CERN, Geneva Switzerland, (2016).

[63] A. Arhrib, Higgs bosons decay into bottom-strange in two Higgs doublets models, Phys. Lett. B 612 (2005) 263 [hep-ph/0409218] [INSPIRE]. 
[64] G. Abbas, A. Celis, X.-Q. Li, J. Lu and A. Pich, Flavour-changing top decays in the aligned two-Higgs-doublet model, JHEP 06 (2015) 005 [arXiv: 1503.06423] [INSPIRE].

[65] A. Greljo, J.F. Kamenik and J. Kopp, Disentangling flavor violation in the top-Higgs sector at the LHC, JHEP 07 (2014) 046 [arXiv: 1404.1278] [INSPIRE].

[66] A. Arhrib, Top and Higgs flavor changing neutral couplings in two Higgs doublets model, Phys. Rev. D 72 (2005) 075016 [hep-ph/0510107] [INSPIRE].

[67] G. Eilam, J.L. Hewett and A. Soni, Rare decays of the top quark in the standard and two Higgs doublet models, Phys. Rev. D 44 (1991) 1473 [INSPIRE].

[68] B. Mele, S. Petrarca and A. Soddu, A new evaluation of the $t \rightarrow c H$ decay width in the Standard Model, Phys. Lett. B 435 (1998) 401 [hep-ph/9805498] [INSPIRE].

[69] J.A. Aguilar-Saavedra, Top flavor-changing neutral interactions: theoretical expectations and experimental detection, Acta Phys. Polon. B 35 (2004) 2695 [hep-ph/0409342] [INSPIRE].

[70] C. Zhang and F. Maltoni, Top-quark decay into Higgs boson and a light quark at next-to-leading order in QCD, Phys. Rev. D 88 (2013) 054005 [arXiv:1305.7386] [INSPIRE].

[71] ATLAS collaboration, Search for flavour-changing neutral current top quark decays $t \rightarrow H q$ in pp collisions at $\sqrt{s}=8 \mathrm{TeV}$ with the ATLAS detector, JHEP 12 (2015) 061 [arXiv: 1509.06047] [INSPIRE].

[72] CMS collaboration, Search for the flavor-changing neutral current decay $t \rightarrow q H$ where the Higgs decays to $b \bar{b}$ pairs at $\sqrt{s}=8 \mathrm{TeV}$, CMS-PAS-TOP-14-020, CERN, Geneva Switzerland, (2014).

[73] CMS collaboration, Search for top quark decays $t \rightarrow q H$ with $H \rightarrow \gamma \gamma$ in pp collisions at $\sqrt{s}=8 \mathrm{TeV}$, CMS-PAS-TOP-14-019, CERN, Geneva Switzerland, (2014).

[74] S. Gori, Three lectures of flavor and CP-violation within and beyond the Standard Model, in 2015 European School of High-Energy Physics (ESHEP 2015), Bansko Bulgaria, 2-15 September 2015 [arXiv: 1610.02629] [INSPIRE].

[75] Top Quark Working Group collaboration, K. Agashe et al., Working group report: top quark, in Community Summer Study 2013: Snowmass on the Mississippi (CSS2013), Minneapolis MN U.S.A., 29 July-6 August 2013 [arXiv:1311.2028] [INSPIRE].

[76] M. Selvaggi, Perspectives for top quark physics at high-luminosity LHC, PoS(TOP2015) 054 [arXiv: 1512.04807] [INSPIRE].

[77] M.L. Mangano et al., Physics at a 100 TeV pp collider: Standard Model processes, arXiv: 1607.01831 [INSPIRE].

[78] W. Altmannshofer, S. Gori and G.D. Kribs, A minimal flavor violating $2 H D M$ at the LHC, Phys. Rev. D 86 (2012) 115009 [arXiv:1210.2465] [INSPIRE].

[79] CMS collaboration, Search for neutral MSSM Higgs bosons decaying into a pair of bottom quarks, JHEP 11 (2015) 071 [arXiv:1506.08329] [INSPIRE].

[80] ATLAS collaboration, Search for minimal supersymmetric Standard Model Higgs bosons $H / A$ in the $\tau \tau$ final state in up to $13.3 \mathrm{fb}^{-1}$ of pp collisions at $\sqrt{s}=13 \mathrm{TeV}$ with the ATLAS detector, ATLAS-CONF-2016-085, CERN, Geneva Switzerland, (2016). 
[81] CMS collaboration, Search for a neutral MSSM Higgs boson decaying into $\tau \tau$ at $13 \mathrm{TeV}$, CMS-PAS-HIG-16-006, CERN, Geneva Switzerland, (2016).

[82] CMS collaboration, Search for a high-mass resonance decaying into a dilepton final state in $13 \mathrm{fb}^{-1}$ of pp collisions at $\sqrt{\mathrm{s}}=13 \mathrm{TeV}$, CMS-PAS-EXO-16-031, CERN, Geneva Switzerland, (2016).

[83] ATLAS collaboration, Search for new high-mass resonances in the dilepton final state using proton-proton collisions at $\sqrt{s}=13 \mathrm{TeV}$ with the ATLAS detector, ATLAS-CONF-2016-045, CERN, Geneva Switzerland, (2016).

[84] ATLAS collaboration, Search for heavy Higgs bosons A/H decaying to a top-quark pair in pp collisions at $\sqrt{s}=8 \mathrm{TeV}$ with the ATLAS detector, ATLAS-CONF-2016-073, CERN, Geneva Switzerland, (2016).

[85] M. Carena, S. Gori, A. Juste, A. Menon, C.E.M. Wagner and L.-T. Wang, LHC discovery potential for non-standard Higgs bosons in the 3 b channel, JHEP 07 (2012) 091 [arXiv: 1203.1041] [INSPIRE].

[86] BABAR collaboration, J.P. Lees et al., Evidence for an excess of $\bar{B} \rightarrow D^{(*)} \tau^{-} \bar{\nu}_{\tau}$ decays, Phys. Rev. Lett. 109 (2012) 101802 [arXiv:1205.5442] [INSPIRE].

[87] BABAR collaboration, J.P. Lees et al., Measurement of an excess of $\bar{B} \rightarrow D^{(*)} \tau^{-} \bar{\nu}_{\tau}$ decays and implications for charged Higgs bosons, Phys. Rev. D 88 (2013) 072012 [arXiv: 1303.0571] [INSPIRE].

[88] LHCb collaboration, Measurement of the ratio of branching fractions $B\left(\bar{B}^{0} \rightarrow D^{*+} \tau^{-} \bar{\nu}_{\tau}\right) / B\left(\bar{B}^{0} \rightarrow D^{*+} \mu^{-} \bar{\nu}_{\mu}\right)$, Phys. Rev. Lett. 115 (2015) 111803 [Addendum ibid. 115 (2015) 159901] [arXiv: 1506.08614] [INSPIRE].

[89] BeLLE collaboration, M. Huschle et al., Measurement of the branching ratio of $\bar{B} \rightarrow D^{(*)} \tau^{-} \bar{\nu}_{\tau}$ relative to $\bar{B} \rightarrow D^{(*)} \ell^{-} \bar{\nu}_{\ell}$ decays with hadronic tagging at Belle, Phys. Rev. D 92 (2015) 072014 [arXiv: 1507.03233] [INSPIRE].

[90] BeLle collaboration, A. Abdesselam et al., Measurement of the branching ratio of $\bar{B}^{0} \rightarrow D^{*+} \tau^{-} \bar{\nu}_{\tau}$ relative to $\bar{B}^{0} \rightarrow D^{*+} \ell^{-} \bar{\nu}_{\ell}$ decays with a semileptonic tagging method, arXiv:1603.06711 [INSPIRE].

[91] A. Abdesselam et al., Measurement of the $\tau$ lepton polarization in the decay $\bar{B} \rightarrow D^{*} \tau^{-} \bar{\nu}_{\tau}$, arXiv: 1608.06391 [INSPIRE].

[92] M. Freytsis, Z. Ligeti and J.T. Ruderman, Flavor models for $\bar{B} \rightarrow D^{(*)} \tau \bar{\nu}$, Phys. Rev. D 92 (2015) 054018 [arXiv: 1506. 08896] [inSPIRE].

[93] F. Mahmoudi and O. Stal, Flavor constraints on the two-Higgs-doublet model with general Yukawa couplings, Phys. Rev. D 81 (2010) 035016 [arXiv:0907.1791] [INSPIRE].

[94] Quark Flavor Physics Working Group collaboration, J.N. Butler et al., Working group report: quark flavor physics, arXiv:1311.1076 [INSPIRE].

[95] A. Bevan et al., Standard Model updates and new physics analysis with the unitarity triangle fit, arXiv:1411.7233 [INSPIRE].

[96] X.-Q. Li, J. Lu and A. Pich, $B_{s, d}^{0} \rightarrow \ell^{+} \ell^{-}$decays in the aligned two-Higgs-doublet model, JHEP 06 (2014) 022 [arXiv: 1404.5865] [INSPIRE].

[97] L. Martini, Search for new physics in the B meson decays: $B_{(s)}^{0} \rightarrow \mu^{+} \mu^{-}$, Nuovo Cim. C 39 (2016) 231 [INSPIRE]. 
[98] C. Bobeth, M. Gorbahn, T. Hermann, M. Misiak, E. Stamou and M. Steinhauser, $B_{s, d} \rightarrow \ell^{+} \ell^{-}$in the Standard Model with reduced theoretical uncertainty, Phys. Rev. Lett. 112 (2014) 101801 [arXiv: 1311.0903] [INSPIRE].

[99] W. Altmannshofer and D.M. Straub, Cornering new physics in $b \rightarrow s$ transitions, JHEP 08 (2012) 121 [arXiv: 1206.0273] [InSPIRE].

[100] W. Altmannshofer, P. Ball, A. Bharucha, A.J. Buras, D.M. Straub and M. Wick, Symmetries and asymmetries of $B \rightarrow K^{*} \mu^{+} \mu^{-}$decays in the Standard Model and beyond, JHEP 01 (2009) 019 [arXiv:0811.1214] [INSPIRE].

[101] LHCb and CMS collaborations, Observation of the rare $B_{s}^{0} \rightarrow \mu^{+} \mu^{-}$decay from the combined analysis of CMS and LHCb data, Nature $\mathbf{5 2 2}$ (2015) 68 [arXiv:1411.4413] [INSPIRE].

[102] ATLAS collaboration, Study of the rare decays of $B_{s}^{0}$ and $B^{0}$ into muon pairs from data collected during the LHC run 1 with the ATLAS detector, Eur. Phys. J. C 76 (2016) 513 [arXiv: 1604.04263] [INSPIRE].

[103] LHCb collaboration, Measurement of the $B_{s}^{0} \rightarrow \mu^{+} \mu^{-}$branching fraction and effective lifetime and search for $B^{0} \rightarrow \mu^{+} \mu^{-}$decays, Phys. Rev. Lett. 118 (2017) 191801 [arXiv: 1703.05747] [INSPIRE].

[104] CMS collaboration, Technical proposal for the phase-II upgrade of the CMS detector, CERN-LHCC-2015-010, CERN, Geneva Switzerland, (2015).

[105] Heavy Flavor Averaging Group (HFAG) collaboration, Y. Amhis et al., Averages of b-hadron, c-hadron and $\tau$-lepton properties as of summer 2014, arXiv:1412.7515 [INSPIRE].

[106] CKMfitTer Group collaboration, J. Charles et al., CP violation and the CKM matrix: assessing the impact of the asymmetric B factories, Eur. Phys. J. C 41 (2005) 1 [hep-ph/0406184] [INSPIRE].

[107] A. Crivellin, A. Kokulu and C. Greub, Flavor-phenomenology of two-Higgs-doublet models with generic Yukawa structure, Phys. Rev. D 87 (2013) 094031 [arXiv:1303.5877] [INSPIRE].

[108] G. Inguglia, Studies of dark sector and B decays involving $\tau$ at Belle and Belle II, PoS (ICHEP2016) 131 [arXiv: 1701.02288] [INSPIRE].

[109] J. Charles, S. Descotes-Genon, Z. Ligeti, S. Monteil, M. Papucci and K. Trabelsi, Future sensitivity to new physics in $B_{d}, B_{s}$ and $K$ mixings, Phys. Rev. D 89 (2014) 033016 [arXiv: 1309.2293] [INSPIRE]. 